\title{
Linear Boltzmann equation as the weak coupling limit of a random Schrödinger equation
}

\section{Citation}

Erdős, László, and Horng\#Tzer Yau. "Linear Boltzmann equation as the weak coupling limit of a random Schrödinger equation." Communications on Pure and Applied Mathematics 53, no. 6 (2000): 667-735.

\section{Published Version}

10.1002/(SICI)1097-0312(200006)53:6<667::AID-CPA1>3.0.C0;2-5

\section{Permanent link}

http://nrs.harvard.edu/urn-3:HUL.InstRepos:32706732

\section{Terms of Use}

This article was downloaded from Harvard University's DASH repository, and is made available under the terms and conditions applicable to Other Posted Material, as set forth at http:// nrs.harvard.edu/urn-3:HUL.InstRepos:dash.current.terms-of-use\#LAA

\section{Share Your Story}

The Harvard community has made this article openly available.

Please share how this access benefits you. Submit a story.

\section{Accessibility}


Jan 061999

\title{
LINEAR BOLTZMANN EQUATION AS THE WEAK COUPLING LIMIT OF A RANDOM SCHRÖDINGER EQUATION
}

\author{
LÁsZló ERdős, HoRng-TzER YAU \\ School of Mathematics, Georgiatech \\ Courant Institute, New York University
}

\begin{abstract}
We study the time evolution of a quantum particle in a Gaussian random environment. We show that in the weak coupling limit the Wigner distribution of the wave function converges to a solution of a linear Boltzmann equation globally in time. The Boltzmann collision kernel is given by the Born approximation of the quantum differential scattering cross section.
\end{abstract}

\section{Introduction.}

Schrödinger equations with random potentials describe the motion of quantum particles in a random environment such as electrons in a semiconductor. The random potential is modeled by a Gaussian random field $V(x)=V_{\omega}(x)$ in $\mathbf{R}^{d}, d \geq 2$, with $\mathbf{E} V(x)=0$ and covariance $G(x-y):=\mathbf{E} V(x) V(y)$. We assume that $G$ is spherically symmetric, smooth and decays fast at infinity. Let $\psi_{t}=\psi_{t, \omega} \in L^{2}\left(\mathbf{R}^{d}\right)$ solve the random Schrödinger equation

$$
i \partial_{t} \psi_{t, \omega}(x)=\left(-\frac{1}{2} \Delta_{x}+\lambda V_{\omega}(x)\right) \psi_{t, \omega}(x)
$$

with a coupling constant $0<\lambda \ll 1$. Here $x, t$ are atomic (microscopic) space and time coordinates.

Our goal is to understand the long time behavior of the random Schrödinger equation (1.1). The first scaling yielding a nontrivial (non-free) evolution is the weak coupling limit

Partially supported by U.S. National Science Foundation grants DMS-9403462, 9703752.

Typeset by $\mathcal{A M S}_{\mathcal{M}}-\mathrm{T}_{\mathrm{E}} \mathrm{X}$ 
which we now describe. Let $\varepsilon$ be the scaling parameter which determines the ratio of the microscopic and macroscopic scales, and let

$$
(X, T):=(x \varepsilon, t \varepsilon)
$$

be the macroscopic coordinates. Note that the velocity is not rescaled, $V=v$. The coupling constant is chosen to scale as

$$
\lambda=\sqrt{\varepsilon} .
$$

This is called the weak coupling limit .

The Gaussian random potential can be replaced with a potential generated by random point obstacles:

$$
V_{\omega}(x)=\sum_{\alpha} V_{0}\left(x-x_{\alpha}(\omega)\right)
$$

where $\left\{x_{\alpha}(\omega)\right\}$ is a random point process (say Poisson) with a density $\varrho$. In this setting, we can consider both the weak coupling limit $\rho=1, \lambda=\sqrt{\varepsilon}$ and the low density limit

$$
\rho=\varepsilon, \lambda=1 .
$$

The model we just described in the low density limit is the quantum analogue of a classical Lorentz gas. It is proved by G. Gallavotti [5], H. Spohn [9] and Boldrighini, Bunimovich and Sinai [1] that the evolution of the phase space density of a classical Lorentz gas converges to a linear Boltzmann equation. For the weak coupling limit, the velocity field under the classical dynamics converges to a Brownian motion on the energy surface, see Kesten and Papanicolaou [7] and Durr, Goldstein and Lebowitz [2].

The picture changes for the quantum dynamics. It was first proved by H. Spohn [8] that for sufficiently small $T$ the weak coupling limit of the quantum dynamics (1.1) is governed by a linear Boltzmann equation. His result was extended to higher order correlation functions by Ho, Landau and Wilkins [6] under the same assumption. Notice that, instead of converging to a Brownian motion, it converges now to a Boltzmann equation. For the low density limit, the quantum Lorentz gas still converges to a Boltzmann equation [3]. In this model the Boltzmann collision kernel $\sigma(U, V)$ is the full quantum scattering cross section. Hence the full quantum collision mechanism is preserved in the scaling limit. In the weak coupling limit only the first Born approximation of the quantum scattering cross section appears in the limiting Boltzmann equation. 
The main objective of this paper is to prove the convergence of the quantum dynamics (1.1) to a Boltzmann equation in the weak coupling limit globally in time. An outline of our approach was given in [3] focusing on point obstacles in the low density limit. This approach was very robust and the point obstacles can be replaced by random fields with certain modifications in the estimates. We choose in this paper the Gaussian law for the random potential only to fix the convention. The approach of $[8,6]$ relies on the convergence of the Duhamel expansion and is valid only for the Gaussian law in short time. Our approach, though still based on Duhamel formula, estimates directly the error terms in the Duhamel formula (rather than expand them to infinity) and the precise tail behavior of the Gaussian law is not used. We shall consider the case $d \geq 2$ in this paper. The case of dimension $d=1$ is different. We do not know the limit equation for the choice of weak coupling limit (1.2) and (1.3). Some logarithmic scaling was considered in a recent preprint [4], obtaining a modified Boltzmann equation in the case the potential of the obstacle is a delta function.

Recall that the linear Boltzmann equation with collision kernel $\sigma(U, V)$ is

$$
\begin{aligned}
\partial_{T} F_{T}(X, V)+V \cdot \nabla_{X} F_{T}(X, V) & =\int\left[\sigma(U, V) F_{T}(X, U)-\sigma(V, U) F_{T}(X, V)\right] d U \\
& =\int \sigma(U, V) F_{T}(X, U) d U-\sigma_{0}(V) F_{T}(X, V)
\end{aligned}
$$

with the total cross section $\sigma_{0}(V):=\int \sigma(V, U) d U$. In case of elastic collision, $\sigma(U, V)$ is supported on the set $\left\{(U, V): U^{2}=V^{2}\right\}$ (onshell condition). If, in addition, the collision mechanism is spherically symmetric, then $\sigma(U, V)=\sigma(U-V)$ and $\sigma_{0}(V)$ depends only on $|V|$.

\subsection{Notations.}

For convenience, we fix a convention to avoid problems with the $(2 \pi)$ 's in the Fourier transform. We define $d x$ to be the Lebesgue measure on $\mathbf{R}^{d}$ divided by $(2 \pi)^{d / 2}$, i.e.

$$
\int d x=\frac{1}{(2 \pi)^{d / 2}} \int d^{*} x,
$$

where the notation $d^{*} x$ is reserved for the genuine $d$-dimensional Lebesgue measure, which will rarely be used. This convention will apply to any space or momentum variables (like $x, y, p, q, r, v$ etc.) in $d$-dimensions, but not to one dimensional integrations (like time variables $s, t$ and their conjugate variables $\alpha, \beta$ etc.), where these symbols still mean the Lebesgue 
measure. With this convention, the $d$-dimensional Fourier transform (usually denoted by hat) is

$$
\widehat{f}(p)=\mathcal{F} f(p):=\int f(x) e^{-i p x} d x
$$

and its inverse

$$
f(x)=\mathcal{F}^{-1} \widehat{f}(x)=\int \widehat{f}(p) e^{i p x} d p .
$$

From this notation is follows that

$$
\int e^{i p x} d x=\delta(p)
$$

(where $\delta(p)$ is understood with respect to the $d p$ measure), while in one dimension

$$
\int_{-\infty}^{\infty} e^{i \alpha t} d \alpha=2 \pi \delta(t)
$$

To avoid confusion, the notation $\int$ is reserved for $\int_{\mathbf{R}^{d}}$, and the limits of integration are always indicated for one dimensional integrals (with Lebesgue measure).

The Wigner transform of a function $\psi \in L^{2}\left(\mathbf{R}^{d}\right)$ is defined as

$$
W_{\psi}(x, v):=\int e^{i \eta x} \overline{\widehat{\psi}\left(v-\frac{\eta}{2}\right)} \widehat{\psi}\left(v+\frac{\eta}{2}\right) d \eta
$$

in position space it is

$$
W_{\psi}(x, v)=\int e^{i v y} \overline{\psi\left(x+\frac{y}{2}\right)} \psi\left(x-\frac{y}{2}\right) d y
$$

Define the rescaled Wigner transform as

$$
W_{\psi}^{\varepsilon}(X, V):=\varepsilon^{-d} W_{\psi}\left(\frac{X}{\varepsilon}, V\right)
$$

Let $J(X, V)$ be a function in the Schwarz space $\mathcal{S}\left(\mathbf{R}^{d} \times \mathbf{R}^{d}\right)$. We need to compute the expectation value of the quantity

$$
\mathcal{O}_{\varepsilon}^{J}:=\left\langle J, W_{\psi_{t}}^{\varepsilon}\right\rangle=\int \overline{J(X, V)} W_{\psi_{t}}^{\varepsilon}(X, V) d X d V
$$

Recall that $X=x \varepsilon, T=t \varepsilon$ and $V=v$. Let $J_{\varepsilon}(x, v):=J(x \varepsilon, v)$ and $\widehat{J}_{\varepsilon}(\xi, v)=\varepsilon^{-d} \widehat{J}\left(\xi \varepsilon^{-1}, v\right)$ denote the Fourier transform in the first variable. Notice that $J_{\varepsilon}(x, v) \neq \varepsilon^{d} J(\varepsilon x, v)$ and $\int J_{\varepsilon}(x, v) d x d v=\varepsilon^{-d}$ with our convention. We can rewrite $\mathcal{O}_{\varepsilon}^{J}$ as

$$
\mathcal{O}_{\varepsilon}^{J}=\int \overline{J(x \varepsilon, v)} W_{\psi_{t}}(x, v) d x d v=\int \overline{J_{\varepsilon}(x, v)} W_{\psi_{t}}(x, v) d x d v
$$


In the Fourier space, it is

$$
\mathcal{O}_{\varepsilon}^{J}=\int \overline{\widehat{J}_{\varepsilon}(\xi, v)} \widehat{W}_{\psi_{t}}(\xi, v) d \xi d v=\varepsilon^{-d} \int \overline{\widehat{J}\left(\xi \varepsilon^{-1}, v\right)} \overline{\widehat{\psi}_{t}\left(v-\frac{\xi}{2}\right)} \widehat{\psi}_{t}\left(v+\frac{\xi}{2}\right) d \xi d v
$$

where we have used the definition (1.5) that

$$
\widehat{W}_{\psi_{t}}(\xi, v)=\overline{\widehat{\psi}_{t}\left(v-\frac{\xi}{2}\right)} \widehat{\psi}_{t}\left(v+\frac{\xi}{2}\right)
$$

Following the usual convention, we use

$$
<x>:=\left(1+x^{2}\right)^{1 / 2} .
$$

Define the following norm for functions on $\mathbf{R}^{d}$

$$
\|f\|_{\alpha, \beta}:=\left\|<x>^{\alpha}<\nabla_{x}>^{\beta} f(x)\right\|_{2} .
$$

\subsection{Main Result.}

Assumption on the potential and the initial function:

We consider a centered real Gaussian field $V(x)$ with covariance $G(x-y):=\mathbf{E} V(x) V(y)$.

Since $G$ is real, $\widehat{G}(p)=\overline{\widehat{G}(-p)}$. From the property of the covariance, $\widehat{G}(p) \geq 0$. Thus we can define $R(p):=\widehat{G}(p)^{1 / 2}$. The covariance of $V$ in the Fourier space can be written as

$$
\mathbf{E} \widehat{V}(p) \widehat{V}(q)=\delta(p+q) \widehat{G}(p)=\delta(p+q) R(p) R(q)
$$

and

$$
\mathbf{E} \widehat{\widehat{V}(p)} \widehat{V}(q)=\delta(p-q) \widehat{G}(p)=\delta(p-q) R(p) R(q)
$$

We assume $R$ to be radially symmetric and smooth. In fact

$$
\|R(p)\|_{20 d, 20 d}<\infty
$$

is enough, but for simplicity we assume $R \in \mathcal{S}\left(\mathbf{R}^{d}\right)$.

We shall choose the initial data

$$
\psi_{0}(x)=\psi_{0}^{\varepsilon}(x):=\varepsilon^{d / 2} h(\varepsilon x) e^{i S(\varepsilon x) / \varepsilon}
$$


with some $h, S \in \mathcal{S}\left(\mathbf{R}^{d}\right)$. We never use the full smoothness and decay properties of the Schwarz space and some weaker conditions such as $\|h\|_{20 d, 20 d}<\infty$ and $\|S\|_{20 d, 20 d}<\infty$ are sufficient. Clearly, $\psi_{0}^{\varepsilon}$ satisfies

$$
\left\|<p>^{20 d} \widehat{\psi}_{0}^{\varepsilon}(p)\right\|_{2} \leq C
$$

with some constant $C$ uniformly in $\varepsilon$. It is well known that

$$
W_{\psi_{0}^{\varepsilon}}^{\varepsilon}(X, V) \rightarrow|h(X)|^{2} \delta(V-\nabla S(X)):=F_{0}(X, V)
$$

weakly in $\mathcal{S}\left(\mathbf{R}^{2 d}\right)$ as $\varepsilon \rightarrow 0$.

Theorem 1.1. Let $\lambda=\sqrt{\varepsilon}, d \geq 2$ and let $\psi_{t, \omega}^{\varepsilon}$ solve the Schrödinger equation (1.1) with initial condition

$$
\psi_{0}^{\varepsilon}(x):=\varepsilon^{d / 2} h(\varepsilon x) \exp \left(\frac{i S(x \varepsilon)}{\varepsilon}\right) .
$$

Assume that $h(X), S(Y)$ are in the Schwarz class. In $d=2$ we assume in addition that the set $\{(X, V): V=0\}$ has zero measure with respect to the probability measure $F_{0}(X, V) d X d V$ (cf. (1.11)). Then for any $T>0$

$$
\mathbf{E} W_{\psi_{T / \varepsilon, \omega}^{\varepsilon}}^{\varepsilon}(X, V) \rightarrow F_{T}(X, V)
$$

weakly in $\mathcal{S}\left(\mathbf{R}^{2 d}\right)$ as $\varepsilon \rightarrow 0$, and $F_{T}$ satisfies the linear Boltzmann equation (1.4) with initial condition $F_{0}(X, V)$ and collision kernel $\sigma(U, V)=4 \pi \widehat{G}(U-V) \delta\left(U^{2}-V^{2}\right)$.

Convention 1. Throughout the paper, $C$ denotes various constants which depend on $d, T, h$, $S$ and $R$ but does not depend on $\varepsilon$.

Convention 2. The random parameter $\omega$ will be omitted from the notation in most cases.

\section{Outline of the Proof.}

\subsection{The Duhamel formula.}

The Duhamel formula states that for any $n_{0} \geq 1$

$$
\psi_{t}=e^{-i t H} \psi_{0}=\sum_{n=0}^{n_{0}-1} \psi_{n}(t)+\Psi_{n_{0}}(t)
$$


where $H_{0}=-\frac{1}{2} \Delta$,

$$
\psi_{n}(t):=(-i)^{n} \lambda^{n} \int_{0}^{t} \ldots \int_{0}^{t}\left(\prod_{j=0}^{n} d s_{j}\right) \delta\left(t-\sum_{j=0}^{n} s_{j}\right) e^{-i s_{0} H_{0}} V e^{-i s_{1} H_{0}} V \ldots V e^{-i s_{n} H_{0}} \psi_{0}
$$

and

$$
\Psi_{n_{0}}(t):=(-i) \lambda \int_{0}^{t} d s e^{-i(t-s) H} V \psi_{n_{0}-1}(s)
$$

is the error term. Sometimes we shall use the notations

$$
\widetilde{\psi}_{n}(t):=\lambda V \psi_{n-1}(t)
$$

and

$$
\psi_{<n_{0}}(t)=\sum_{n=0}^{n_{0}-1} \psi_{n}(t)
$$

We shall choose

$$
n_{0}=n_{0}(\varepsilon)=\frac{\gamma|\log \varepsilon|}{\log |\log \varepsilon|}
$$

with some small fixed $\gamma>0$. Notice that

$$
\varepsilon^{\alpha}|\log \varepsilon|^{n_{0}(\varepsilon)} \rightarrow 0 \quad \text { and } \quad \varepsilon^{\alpha}\left[n_{0}(\varepsilon)\right] ! \rightarrow 0
$$

as $\varepsilon \rightarrow 0$ for any $\alpha>\gamma$.

For any subset $I \subset \mathbf{N}$, we use the convention that

$$
\mathbf{p}_{I}:=\left\{p_{i}: i \in I\right\}
$$

We also introduce the infinite sequence

$$
\mathbf{p}:=\left\{p_{0}, p_{1}, \ldots\right\}
$$

In the special case

$$
I_{n}:=\{0,1, \ldots, n\}
$$

we denote $\mathbf{p}_{n}:=\mathbf{p}_{I_{n}}$. Denote $\mathbf{p}_{n, \widehat{0}}:=\mathbf{p}_{I_{n} \backslash\{0\}}$. Define the kernel for the free evolution in the Fourier (momentum) space by

$$
K(t ; \mathbf{p}, I):=K\left(t ; \mathbf{p}_{I}\right)=(-i)^{|I|-1} \int_{0}^{t}\left(\prod_{j \in I} d s_{j}\right) \delta\left(t-\sum_{j \in I} s_{j}\right) \prod_{j \in I} e^{-i s_{j} p_{j}^{2} / 2}
$$


Again, we denote the special case $I=I_{n}$ by

$$
K(t ; \mathbf{p}, n):=K\left(t ; \mathbf{p}, I_{n}\right)
$$

Denote the contribution from the potential term by

$$
L(\mathbf{p}, n):=\prod_{j=0}^{n-1} \widehat{V}\left(p_{j}-p_{j+1}\right)
$$

Then we can rewrite the $n$-th order wave function as

$$
\widehat{\psi}_{n}\left(t, p_{0}\right)=\lambda^{n} \int d \mathbf{p}_{n, \widehat{0}} K(t ; \mathbf{p}, n) L(\mathbf{p}, n) \widehat{\psi}_{0}\left(p_{n}\right)
$$

The decomposition (2.1) has the disadvantage that the threshold $n_{0}$ depends on the scaling parameter. Hence, we rewrite it as

$$
\psi_{t}=\sum_{n=0}^{M-1} \psi_{n}(t)+\sum_{n=M}^{n_{0}-1} \psi_{n}(t)+\Psi_{n_{0}}(t)=\psi_{t}^{\text {main }}+\psi_{t}^{\text {error }}
$$

where

$$
\psi_{t}^{\text {main }}=\psi_{t, M}^{\text {main }}:=\sum_{n=0}^{M-1} \psi_{n}(t)
$$

and

$$
\psi_{t}^{\text {error }}=\psi_{t, M}^{\text {error }}:=\sum_{n=M}^{n_{0}-1} \psi_{n}(t)+\Psi_{n_{0}}(t) .
$$

We shall show in Sections 3. and 5. that for any $T>0$

$$
\lim _{M \rightarrow \infty} \lim _{\varepsilon \rightarrow 0} \mathbf{E}\left\|\psi_{T / \varepsilon, M}^{\text {error }}\right\|^{2}=0
$$

From the unitarity of the Schrödinger evolution, $\left\|\widehat{\psi}_{t}\right\|^{2}=1$ for all $t$. Thus $\left\|\widehat{\psi}^{\text {main }}\right\|^{2}$ is bounded from the inequality

$$
\left\|\widehat{\psi}_{t}^{\text {main }}\right\|^{2} \leq 2\left\|\widehat{\psi}_{t}\right\|^{2}+2\left\|\widehat{\psi}_{t}^{\text {error }}\right\|^{2}
$$

We shall consider test functions $\Gamma=\Gamma_{\varepsilon}$ in Fourier space such that $\Gamma_{\varepsilon}(\xi, v) \in L_{\xi}^{1}\left(\mathbf{R}^{d}, L_{v}^{\infty}\left(\mathbf{R}^{d}\right)\right)$ uniformly in $\varepsilon$, i.e.

$$
\sup _{0<\varepsilon \leq 1} \int \sup _{v}\left|\Gamma_{\varepsilon}(\xi, v)\right| d \xi<\infty .
$$


In particular $\Gamma_{\varepsilon}=\widehat{J}_{\varepsilon}$ satisfies (2.8). The expected value of the observable generated by a test function in this class is bounded in the sense that

$$
\left|\mathbf{E} \int \overline{\Gamma(\xi, v)} \widehat{W}_{\psi}(\xi, v) d \xi d v\right| \leq C\left(\int \sup _{v}|\Gamma(\xi, v)| d \xi\right) \mathbf{E}\|\psi\|^{2} .
$$

To prove this, we have from the Schwarz inequality and (1.7)

$$
\begin{aligned}
\left|\mathbf{E} \int \overline{\Gamma(\xi, v)} \widehat{W}_{\psi}(\xi, v) d \xi d v\right| & \leq \mathbf{E} \int d \xi d v|\Gamma(\xi, v)|\left(\left|\widehat{\psi}\left(v-\frac{\xi}{2}\right)\right|^{2}+\left|\widehat{\psi}\left(v+\frac{\xi}{2}\right)\right|^{2}\right) \\
& \leq C\left(\int \sup _{v}|\Gamma(\xi, v)| d \xi\right) \mathbf{E}\|\widehat{\psi}\|^{2}
\end{aligned}
$$

We can generalize this inequality to prove the continuity of the expected value of the observables with respect to the decomposition of the wave function in $L^{2}$. Suppose that we have a decomposition of a wave function $\psi=\psi_{1}+\psi_{2}$. Then

$$
\left|\mathbf{E} \mathcal{O}_{\varepsilon}^{J}-\mathbf{E} \int \overline{\widehat{J}_{\varepsilon}(\xi, v)} \widehat{W}_{\psi_{1}}(\xi, v) d \xi d v\right|=\left|\mathbf{E} \int \overline{\widehat{J}_{\varepsilon}(\xi, v)}\left[\widehat{W}_{\psi}(\xi, v)-\widehat{W}_{\psi_{1}}(\xi, v)\right] d \xi d v\right|
$$

From the Schwarz inequality and (1.7)

$$
\begin{aligned}
& \left|\widehat{W}_{\psi}(\xi, v)-\widehat{W}_{\psi_{1}}(\xi, v)\right| \\
& \leq C\left(\alpha\left|\widehat{\psi}_{1}\left(v-\frac{\xi}{2}\right)\right|^{2}+\alpha\left|\widehat{\psi}_{1}\left(v+\frac{\xi}{2}\right)\right|^{2}+\alpha^{-1}\left|\widehat{\psi}_{2}\left(v-\frac{\xi}{2}\right)\right|^{2}+\alpha^{-1}\left|\widehat{\psi}_{2}\left(v+\frac{\xi}{2}\right)\right|^{2}\right) .
\end{aligned}
$$

Optimizing $\alpha$, we have

$$
\left|\mathbf{E} \mathcal{O}_{\varepsilon}^{J}-\mathbf{E} \int \overline{\widehat{J}_{\varepsilon}(\xi, v)} \widehat{W}_{\psi_{1}}(\xi, v) d \xi d v\right| \leq C\left(\sup _{\varepsilon \leq 1} \int \sup _{v}\left|\widehat{J}_{\varepsilon}(\xi, v)\right| d \xi\right) \sqrt{\mathbf{E}\left\|\psi_{1}\right\|^{2} \mathbf{E}\left\|\psi_{2}\right\|^{2}} .
$$

We use this for $\psi_{1}=\psi_{t}^{\text {main }}, \psi_{2}=\psi_{t}^{\text {error }}$. We already know from (2.7) that the $L^{2}$ norm of the error terms vanishes. Therefore,

$$
\lim _{\varepsilon \rightarrow 0} \mathbf{E} \mathcal{O}_{\varepsilon}^{J}=\lim _{M \rightarrow \infty} \lim _{\varepsilon \rightarrow 0} \mathbf{E} \int{\widehat{J_{\varepsilon}}(\xi, v)}_{W_{\psi_{T / \varepsilon, M}^{\text {main }}}}(\xi, v) d \xi d v
$$

So we only have to compute the Wigner distribution of the main term in the limit. This will be done in Section 4 . 


\subsection{Pairings.}

We shall compute the expectation of the Wigner transform. Define

$$
U_{n, n^{\prime}}^{\Gamma}(t):=\mathbf{E} \int \overline{\Gamma(\xi, v)} \widehat{\psi}_{n}\left(t, v-\frac{\xi}{2}\right) \widehat{\psi}_{n^{\prime}}\left(t, v+\frac{\xi}{2}\right) d v d \xi
$$

for any $n, n^{\prime} \geq 0$ and any testfunction $\Gamma(\xi, v) \in L_{\xi}^{1}\left(\mathbf{R}^{d}, L_{v}^{\infty}\left(\mathbf{R}^{d}\right)\right)\left(\right.$ i.e. $\left.\int \sup _{v}|\Gamma(\xi, v)| d \xi<\infty\right)$. From (1.6) we have formally

$$
\lim _{\varepsilon \rightarrow 0} \mathbf{E} \mathcal{O}_{\varepsilon}^{J} \sim \sum_{n, n^{\prime}=0}^{\infty} \lim _{\varepsilon \rightarrow 0} U_{n, n^{\prime}}^{\widehat{J_{\varepsilon}}}(T / \varepsilon)
$$

Note that if $\Gamma(\xi, v)=\delta(\xi)$ then

$$
U_{n, n}(t):=U_{n, n}^{\Gamma=\delta(\xi)}(t)=\mathbf{E}\left\|\psi_{n}(t)\right\|^{2}
$$

is simply the expectation of the $L^{2}$ norm of $\psi_{n}$. Recall $J_{\varepsilon}(x, v):=J(x \varepsilon, v), \widehat{J}_{\varepsilon}(\xi, v)=$ $\varepsilon^{-d} \widehat{J}\left(\xi \varepsilon^{-1}, v\right)$ and certainly $\widehat{J}_{\varepsilon}(\xi, v) \in L_{\xi}^{1}\left(\mathbf{R}^{d}, L_{v}^{\infty}\left(\mathbf{R}^{d}\right)\right)$ uniformly in $\varepsilon$.

By definition of $\widehat{\psi}_{n}(2.6), U_{n, n^{\prime}}^{\Gamma}$ has two sets of potentials with the following arguments:

$$
\begin{gathered}
\lambda^{n} \overline{\widehat{V}\left(p_{0}-p_{1}\right) \widehat{V}\left(p_{1}-p_{2}\right)} \ldots \overline{\widehat{V}\left(p_{n-1}-p_{n}\right)} \\
\lambda^{n^{\prime}} \widehat{V}\left(p_{0}^{\prime}-p_{1}^{\prime}\right) \widehat{V}\left(p_{1}^{\prime}-p_{2}^{\prime}\right) \ldots \widehat{V}\left(p_{n^{\prime}-1}^{\prime}-p_{n^{\prime}}^{\prime}\right)
\end{gathered}
$$

with $p_{0}:=v-\xi / 2, p_{0}^{\prime}:=v+\xi / 2$. Since $V$ is Gaussian, we can compute the expectation of $U_{n, n^{\prime}}^{\Gamma}$ explicitly. This introduces pairings of potentials and yields delta functions among the momenta. Clearly, $U_{n, n^{\prime}}^{\Gamma}=0$ if $n+n^{\prime}$ is odd. We divide the delta functions into the following three types:

$$
\begin{array}{rlr}
\text { Type } I: & \delta\left(-p_{i}+p_{i+1}-p_{j}+p_{j+1}\right) \quad i<j \\
\text { Type } I^{\prime}: & \delta\left(p_{i}^{\prime}-p_{i+1}^{\prime}+p_{j}^{\prime}-p_{j+1}^{\prime}\right) \quad i<j \\
\text { Type } I I: & \delta\left(-p_{i}+p_{i+1}+p_{j}^{\prime}-p_{j+1}^{\prime}\right) .
\end{array}
$$

In all these delta functions, each $p_{i}$ and $p_{i}^{\prime}$ with $0<i<n, 0<i^{\prime}<n^{\prime}$ appear exactly twice with opposite signs, $p_{0}^{\prime}$ and $p_{n}$ once with plus sign, and $p_{0}$ and $p_{n^{\prime}}^{\prime}$ once with a minus sign. Finally, the relation $p_{0}=v-\xi / 2, p_{0}^{\prime}=v+\xi / 2$ is expressed by $\delta\left(p_{0}-p_{0}^{\prime}+\xi\right)$, i.e. we add

$$
\text { Type } I I I(\xi): \delta\left(p_{0}-p_{0}^{\prime}+\xi\right)
$$


to our list. In particular, $p_{0}=p_{0}^{\prime}$ if $\xi=0$, which is needed for computing the $L^{2}$-norm. We have exactly $\frac{n+n^{\prime}}{2}+1=\bar{n}+1$ delta functions with $\bar{n}:=\frac{n+n^{\prime}}{2}$. Notice that if add up the arguments of all these delta functions, we arrive at a new delta function $\delta\left(p_{n}-p_{n^{\prime}}^{\prime}+\xi\right)$. We can replace the delta function $\delta\left(p_{0}-p_{0}^{\prime}+\xi\right)$ by the new delta funtion $\delta\left(p_{n}-p_{n^{\prime}}^{\prime}+\xi\right)$.

Define

$$
F(\mathbf{p}, n):=\prod_{j=0}^{n-1} R\left(p_{j}-p_{j+1}\right) \widehat{\psi}_{0}\left(p_{n}\right)
$$

and let

$$
\Phi(p):=<p>^{20 d}\left|\widehat{\psi}_{0}(p)\right| .
$$

By the property of the initial wave function (1.10), we have $\Phi \in L^{2}$.

Let $\Pi_{n, n^{\prime}}$ denote the set of all pairing with $n$ copies of $\bar{V}$ and $n^{\prime}$ copies of $V$. Denote by $\Delta_{\pi}\left(\mathbf{p}, \mathbf{p}^{\prime}\right)$ the product of Type I, I', and II delta functions associated with the pairing $\pi$. With these notations, we have

$$
U_{n, n^{\prime}}^{\Gamma}=\mathbf{E} \iint d \xi d v \overline{\Gamma(\xi, v)} \overline{\widehat{\psi}_{n}\left(t, v-\frac{\xi}{2}\right)} \widehat{\psi}_{n^{\prime}}\left(t, v+\frac{\xi}{2}\right)=\sum_{\pi \in \Pi_{n, n^{\prime}}} C_{\pi}^{\Gamma}
$$

with

$$
\begin{aligned}
C_{\pi}^{\Gamma}:= & \lambda^{n+n^{\prime}} \int d \xi d v \overline{\Gamma(\xi, v)} \int d \mathbf{p}_{n} d \mathbf{p}_{n^{\prime}}^{\prime} \overline{K(t, \mathbf{p}, n)} K\left(t, \mathbf{p}^{\prime}, n^{\prime}\right) \\
& \times \Delta_{\pi}\left(\mathbf{p}, \mathbf{p}^{\prime}\right) \overline{F(\mathbf{p}, n)} F\left(\mathbf{p}^{\prime}, n^{\prime}\right) \delta\left(p_{0}-v+\xi / 2\right) \delta\left(p_{0}^{\prime}-v-\xi / 2\right) .
\end{aligned}
$$

Here $F$ is defined in (2.13). In the special case that $\Gamma$ is just the delta function in $\xi$, we drop the superscript $\Gamma$. This is needed when we estimate the $L^{2}$ norm. We shall use the notation

$$
\Delta_{\pi}^{\xi}\left(\mathbf{p}, \mathbf{p}^{\prime}\right):=\Delta_{\pi}\left(\mathbf{p}, \mathbf{p}^{\prime}\right) \delta\left(p_{0}-p_{0}^{\prime}+\xi\right)=\Delta_{\pi}\left(\mathbf{p}, \mathbf{p}^{\prime}\right) \delta\left(p_{0}-p_{n^{\prime}}^{\prime}+\xi\right)
$$

Notice that the product of all delta functions in (2.16) is $\Delta_{\pi}^{\xi}\left(\mathbf{p}, \mathbf{p}^{\prime}\right) \delta\left(p_{0}-v+\xi / 2\right)$. When computing $L^{2}$-norm, we shall need $\Delta_{\pi}^{0}$ i.e. $\xi=0$. Therefore,

$$
C_{\pi}:=\lambda^{n+n^{\prime}} \int d \mathbf{p}_{n} d \mathbf{p}_{n^{\prime}}^{\prime} \overline{K(t, \mathbf{p}, n)} K\left(t, \mathbf{p}^{\prime}, n^{\prime}\right) \Delta_{\pi}^{0}\left(\mathbf{p}, \mathbf{p}^{\prime}\right) \overline{F(\mathbf{p}, n)} F\left(\mathbf{p}^{\prime}, n^{\prime}\right) .
$$

Next, we describe the structure of $\Delta_{\pi}^{\xi}\left(\mathbf{p}, \mathbf{p}^{\prime}\right)$. Sometimes we shall consider the two sets of momenta in a unified way, hence we define

$$
w_{j}:=p_{n-j}, \quad 0 \leq j \leq n ; \quad w_{n+j+1}:=p_{j}^{\prime}, \quad 0 \leq j \leq n^{\prime}
$$

Denote by $\mathbf{w}=\left(w_{0}, w_{1}, w_{2}, \ldots\right)$. We shall use the $\left(\mathbf{p}_{n}, \mathbf{p}_{n^{\prime}}^{\prime}\right)$ and the $\mathbf{w}_{2 \bar{n}+1}$ notations simultaneously where $2 \bar{n}=n+n^{\prime}$. 
Definition 2.1. We consider products of delta functions with arguments that are linear combinations of the momenta $\left\{w_{0}, w_{1}, \ldots, w_{2 \bar{n}+1}\right\}$. Two products of such delta functions are called equivalent if they determine the same affine subspace of $\mathbf{R}^{2 \bar{n}+2}=\left\{\left(w_{0}, w_{1}, \ldots, w_{2 \bar{n}+1}\right)\right\}$. The rank of a product of deltafunctions is the codimension of this affine subspace.

One can obtain new delta functions from the given ones, by taking linear combinations of their arguments. In particular, we can obtain identifications of momenta.

Definition 2.2. The product of delta functions $\Delta_{\pi}^{\xi}(\mathbf{w})$ forces a new delta function $\delta\left(\sum_{j} a_{j} w_{j}\right)$, if $\sum_{j} a_{j} w_{j}=0$ is an identity in the affine subspace determined by $\Delta_{\pi}^{\xi}(\mathbf{w})$. If $\Delta_{\pi}^{\xi}(\mathbf{w})$ forces $\delta\left(w_{i}-w_{j}\right)$, with some $i, j$, then we say it forces the identification $w_{i}=w_{j}$.

Lemma 2.3 . (I) For every pairing $\pi$, there exists a partition $A \cup B=\{0,1,2, \ldots 2 \bar{n}, 2 \bar{n}+1\}$, $A \cap B=\emptyset$ such that

(i) $|A|=|B|=\bar{n}+1,0 \in A, 2 \bar{n}+1 \in B$,

(ii) $\Delta_{\pi}^{\xi}\left(\mathbf{p}, \mathbf{p}^{\prime}\right)=\Delta_{\pi}^{\xi}(\mathbf{w})$ is equivalent to the product of the following delta functions:

$$
\delta\left(w_{j}-\sum_{\substack{\ell \in A \\ \ell<j}} \pm w_{\ell}\right) \quad \forall j \in B, j \neq 2 \bar{n}+1
$$

and $\quad \delta\left(w_{0}-w_{2 \bar{n}+1}+\xi\right)$.

In other words, we have

$$
\mathbf{w}_{B}=\mathcal{M}_{0} \mathbf{w}_{A}+\xi \mathbf{e}_{1}
$$

where $\mathbf{w}_{A}:=\left(w_{\ell}: \ell \in A\right)^{T}, \mathbf{w}_{B}:=\left(w_{j}: j \in B\right)^{T}$ and $\mathbf{e}:=(0,0, \ldots, 0,1)^{T}$. Here $\mathcal{M}_{0}=\mathcal{M}_{0}^{(\pi)}$ is an $(\bar{n}+1) \times(\bar{n}+1)$ matrix, such that all its entries are 0 or \pm 1 and each row contains one more +1 than -1 . In particular, each row has an odd number of nonzero elements. We can order the elements in $B$ by $B=\left\{b_{1}<\ldots<b_{\nu}<\ldots<b_{\bar{n}+1}\right\}$, and similarly for $A=\left\{a_{1}<\ldots<a_{\mu}<\ldots<a_{\bar{n}+1}\right\}$. Then the matrix $\mathcal{M}_{0}=\left(m_{\nu \mu}\right)$ has the property that $m_{\nu \mu}=0$ if $a_{\mu}>b_{\nu}$. The rank of $\Delta_{\pi}^{\xi}(\mathbf{w})$ is $\bar{n}+1$.

(II) Let $\widetilde{\Delta} \xi\left(\mathbf{p}, \mathbf{p}^{\prime}\right)$ be the product of a subset of delta functions in $\Delta_{\pi}^{\xi}\left(\mathbf{p}, \mathbf{p}^{\prime}\right)$, which contains $r \leq \bar{n}$ delta functions. We also assume that $\delta\left(p_{n}-p_{n^{\prime}}^{\prime}+\xi\right)$ is forced by $\widetilde{\Delta} \xi\left(\mathbf{p}, \mathbf{p}^{\prime}\right)$. Then the sets $A, B$ can be chosen exactly with the properties above, except that $|B|=r$ and $|A|=2 \bar{n}+2-r$. The dimension of $\mathcal{M}_{0}$ is $r \times(2 \bar{n}-r+2)$ but all the other properties of $\mathcal{M}_{0}$ are the same. The rank of $\widetilde{\Delta_{\pi}^{\xi}}\left(\mathbf{p}, \mathbf{p}^{\prime}\right)$ is $r$.

Remark. By definition of $B$, the last line of $(2.19)$ is $w_{2 \bar{n}+1}=w_{0}+\xi\left(\right.$ recall $\left.b_{\bar{n}+1}=2 \bar{n}+1\right)$. This is the sum of the arguments of all delta functions in $\Delta_{\pi}^{\xi}(\mathbf{w})$. 
The proof of Lemma 2.3 is based upon a simple Gaussian elimination. From the coefficients in the arguments of the delta functions in $\Delta_{\pi}^{\xi}(\mathbf{w})$ we form an $(\bar{n}+1) \times(2 \bar{n}+1)$ matrix. Each row of this matrix corresponds to a delta function, and each column corresponds to a momentum $w_{k}, k=0,1, \ldots 2 \bar{n}+1$. The set $B$ consists of the biggest indices that appear among the arguments in each delta function. The complementary set is $A$. The corresponding momenta are called $B$ - and $A$-momenta, respectively. Then we perform a Gaussian elimination to express the $B$-momenta as linear combinations of the $A$-momenta. It is easy to show that, after the elimination, the matrix has the structure $\left[\mathcal{M}_{0} \mid-I\right]$ with a matrix $\mathcal{M}_{0}$ described in the Lemma. The details are given in the Appendix.

\subsection{Graphs.}

We classify the pairings according to their combinatorial structure. This classification and certain properties of the pairings are easier to illustrate in a graphical representation. For any pairing $\pi \in \Pi_{n, n^{\prime}}$ we define a graph $G=G_{\pi}$ as follows (Fig. 1). There are $n+n^{\prime}$ bullets arranged in two lines. They represent the potentials in $\psi_{n}$ and $\psi_{n^{\prime}}$. The upper line correspond to $\overline{\widehat{\psi}_{n}}$, the lower line to $\widehat{\psi}_{n^{\prime}}$. The initial wave function $\overline{\widehat{\psi}_{0}\left(p_{n}\right)} \widehat{\psi}_{0}\left(p_{n^{\prime}}^{\prime}\right)$ is denoted by a small square on the right side of the picture. The observable

$$
\int d \xi d v \Gamma(\xi, v) \delta\left(p_{0}-v+\xi / 2\right) \delta\left(p_{0}^{\prime}-v-\xi / 2\right)=\Gamma\left(p_{0}^{\prime}-p_{0},\left(p_{0}+p_{0}^{\prime}\right) / 2\right)
$$

is denoted by a small square on the left. The bullets are joined by directed arrows representing momenta; $p_{1}, \ldots p_{n-1}, p_{1}^{\prime}, \ldots p_{n^{\prime}-1}^{\prime}$. The squares are connected with the first and last bullets by arrows corresponding to $p_{0}, p_{0}^{\prime}, p_{n}, p_{n^{\prime}}^{\prime}$ as shown on Fig. 1. The arrows are called momentum lines, denoted by $e\left(p_{i}\right)$ and $e\left(p_{i}^{\prime}\right)$, the argument referring to the corresponding momentum. The bullets and the squares with the arrows form a directed circle graph, denoted by $C$.

Type I, I' and II delta functions are represented by undirected dashed lines joining two bullets and we call them pairing lines. We draw these lines outside of $C$. For example, Fig 1. represents a pairing $\pi \in \Pi_{6,4}$ with the following deltafunctions: $\delta\left(-p_{0}+p_{1}-p_{2}+p_{3}\right)$, $\delta\left(-p_{1}+p_{2}-p_{3}+p_{4}\right), \delta\left(-p_{4}+p_{5}+p_{3}^{\prime}-p_{4}^{\prime}\right), \delta\left(-p_{5}+p_{6}+p_{3}^{\prime}-p_{2}^{\prime}\right)$ and $\delta\left(p_{0}^{\prime}-p_{1}^{\prime}+p_{1}^{\prime}-p_{2}^{\prime}\right)$. Notice that the signs of the momenta in the arguments correspond to the direction of the arrows. At any bullet, an incoming arrow involves a plus sign, an outgoing one involves a minus sign in the delta function pairing that bullet. 


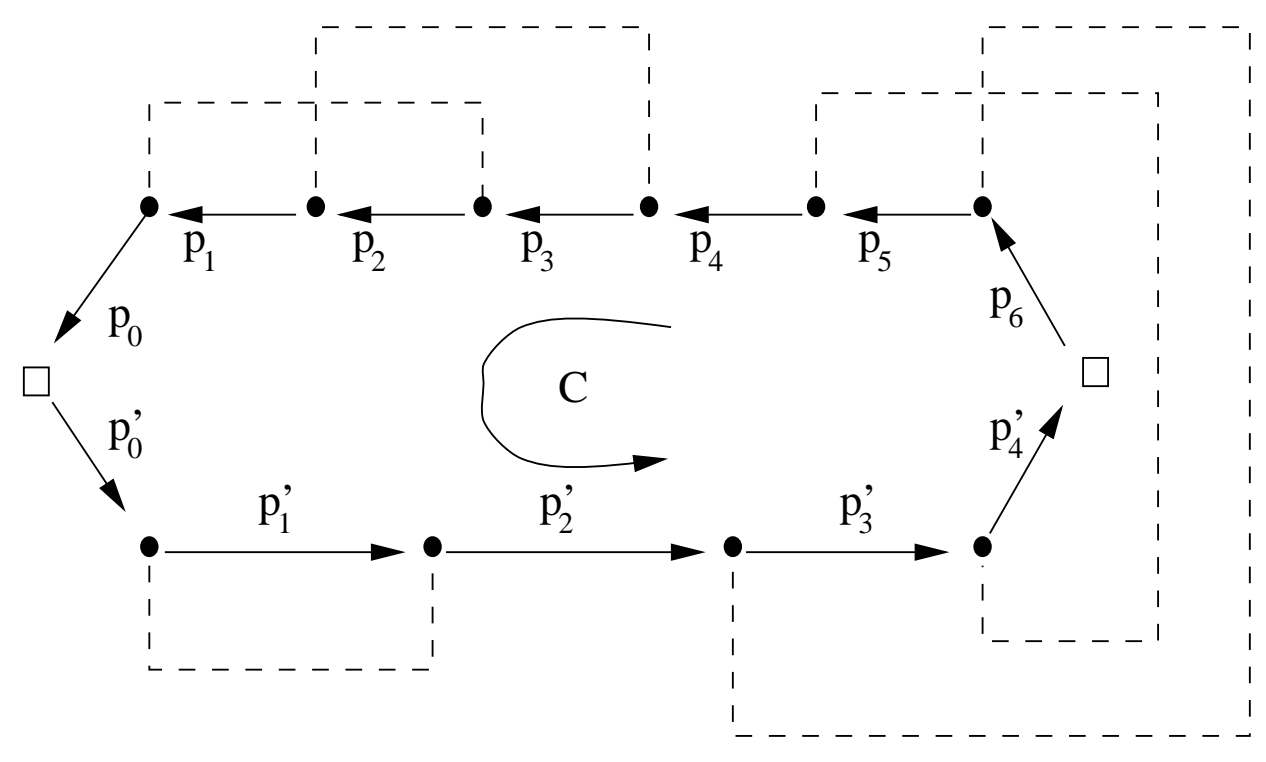

Fig. 1

Type $\operatorname{III}(\xi)$ delta function $\delta\left(p_{0}-p_{0}^{\prime}+\xi\right)$ corresponds to a momentum transfer $\xi$ at the left square. Recall that by adding up the arguments of all the delta functions, we arrive at $\delta\left(p_{n}-p_{n^{\prime}}^{\prime}+\xi\right)$, implying a momentum transfer $\xi$ at the right square as well. The actual value $\xi$ of these momentum transfers will not play any role in the definitions and theorems related to the graphical representations. We can set $\xi=0$ for simplicity. The classification of the pairings will depend only on Type I, I', II delta functions.

Sometimes we use the notation $w_{0}, w_{1}, \ldots, w_{2 \bar{n}+1}$ for labelling the momentum lines. The momentum and pairing lines together are called the edges of $G_{\pi}$, while the bullets and squares are called the vertices of $G_{\pi}$. The set of edges of a graph $G$ is $E(G)$, the set of vertices is $V(G)$. We introduce the following classes of pairings:

Definition 2.4. The pairing $\pi \in \Pi_{n, n^{\prime}}$ is called crossing if there exist two delta functions among Type I, I' and II; $\delta\left(w_{i}-w_{i+1}+w_{j}-w_{j+1}\right)$ and $\delta\left(w_{k}-w_{k+1}+w_{\ell}-w_{\ell+1}\right)$ such that $i<k<j<\ell$. Otherwise, the pairing is called noncrossing.

Definition 2.5. A noncrossing pairing is called nested if there are two Type I delta functions $\delta\left(-p_{i}+p_{i+1}-p_{j}+p_{j+1}\right), \delta\left(-p_{k}+p_{k+1}-p_{u}+p_{u+1}\right)$ with $i<k<u<j$ or there are two Type 
I' delta functions $\delta\left(p_{i}^{\prime}-p_{i+1}^{\prime}+p_{j}^{\prime}-p_{j+1}^{\prime}\right), \delta\left(p_{k}^{\prime}-p_{k+1}^{\prime}+p_{u}^{\prime}-p_{u+1}^{\prime}\right)$ with $i^{\prime}<k^{\prime}<u^{\prime}<j^{\prime}$. A noncrossing and nonnested pairing is called simple.

Remark. These notions could be defined directly by using the graph of a pairing. Obviously, $\pi$ is crossing if and only if it is not possible to draw all the pairing lines in $G_{\pi}$ without crossings. Recall that the pairing lines are always drawn outside $C$. Similarly, a noncrossing $\pi$ is nested if and only if $G_{\pi}$ contains two Type I (or two Type I') pairing lines such that one is fully inside the other. These pairing lines are called outer and inner deltas. The inner delta $\delta\left(-p_{k}+p_{k+1}-p_{u}+p_{u+1}\right)$ is called minimal if $u=k+1$. Fig. 2 shows a nest with Type I delta functions.

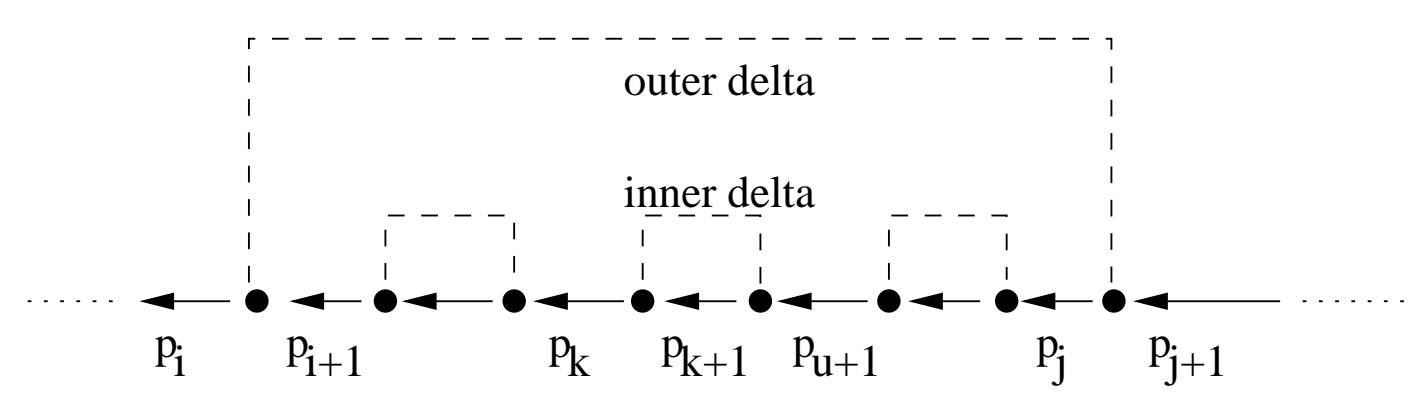

Fig. 2

The inner delta is minimal, i.e. $\mathrm{u}=\mathrm{k}+1$.

Recall the Definition 2.2 of the forced identification. The following Lemma gives a characterization of the forced identifications in terms of the graph:

Lemma 2.6 . The product of delta functions $\Delta_{\pi}^{0}(\mathbf{w})$ forces $w_{i}=w_{j}$, for some $0 \leq i, j \leq$ $2 \bar{n}+1$, if and only if the removal of the two momentum lines corresponding to $w_{i}, w_{j}$ makes the graph $G_{\pi}$ disconnected.

The proof of Lemma 2.6 is given in the Appendix. Observe that this Lemma properly contains the identifications $p_{0}=p_{0}^{\prime}$ and $p_{n}=p_{n^{\prime}}^{\prime}$, as the removal of one of these pairs of momentum lines makes one of the squares disconnected. Finally, the relation between the graphical and matrix representation of the crossing pairing is given in the following lemma which will be proved in the appendix. Recall the construction of the matrix $\mathcal{M}_{0}^{(\pi)}$ in the proof of Lemma 2.3.

Lemma 2.7 . A pairing $\pi \in \Pi_{n, n^{\prime}}$ is crossing if and only if there is a row in the matrix $\mathcal{M}_{0}^{(\pi)}$ which has more than one nonzero element. 


\section{3. $L^{2}$ Estimates of the Duhamel formula.}

We first fix some notations. The following property can be easily checked

$$
\frac{1}{<p_{n}>^{2 d}} \prod_{j=0}^{n-1} \frac{1}{\left.<p_{j}-p_{j+1}\right\rangle^{2 d}} \leq \frac{C^{n}}{\left.<p_{k}\right\rangle^{2 d}}
$$

for any $0 \leq k \leq n$. Hence

$$
\frac{1}{\left.<p_{n}\right\rangle^{20 d}} \prod_{j=0}^{n-1} \frac{1}{\left.<p_{j}-p_{j+1}\right\rangle^{20 d}} \leq C^{n} \prod_{i=1}^{9} \frac{1}{\left\langle p_{\ell_{i}}\right\rangle^{2 d}} \prod_{j=0}^{n-1} \frac{1}{\left\langle p_{j}-p_{j+1}\right\rangle^{2 d}}
$$

for any numbers $\ell_{1}, \ell_{2}, \ldots \ell_{9}$ between 0 and $n$. It is impossible to have the product of all factors, $\prod_{j}<p_{j}>^{-2 d}$, but one has the freedom to choose some of them as we did in (3.2). By the assumption (1.9) on $R$ and the last inequality, $F$ satisfies

$$
|F(\mathbf{p}, n)| \leq \frac{C^{n} \Phi\left(p_{n}\right)}{<p_{n}>^{20 d}} \prod_{j=0}^{n-1} \frac{1}{\left.<p_{j}-p_{j+1}\right\rangle^{20 d}} \leq C^{n} \Phi\left(p_{n}\right) \prod_{i=1}^{9} \frac{1}{\left\langle p_{\ell_{i}}\right\rangle^{2 d}} \prod_{j=0}^{n-1} \frac{1}{\left.<p_{j}-p_{j+1}\right\rangle^{2 d}}
$$

for any $\ell_{1}, \ell_{2}, \ldots, \ell_{9} \leq n$.

Define the measure

$$
d \mu\left(\mathbf{p}_{n}\right):=\frac{\left|\Phi\left(p_{n}\right)\right|^{2} d \mathbf{p}_{n}}{<p_{n}>^{20 d}} \prod_{j=0}^{n-1} \frac{1}{<p_{j}-p_{j+1}>^{20 d}},
$$

or, more generally for any set $I:=\left\{i_{1}, i_{2}, \ldots i_{n}\right\}$

$$
d \mu\left(\mathbf{p}_{I}\right):=\frac{\left|\Phi\left(p_{i_{n}}\right)\right|^{2} d \mathbf{p}_{I}}{<p_{i_{n}}>^{20 d}} \prod_{j=0}^{n-1} \frac{1}{<p_{i_{j}}-p_{i_{j+1}}>^{20 d}} .
$$

To estimate this measure we shall recall (3.2) yielding

$$
d \mu\left(\mathbf{p}_{n}\right) \leq \frac{C^{n}\left|\Phi\left(p_{n}\right)\right|^{2} d \mathbf{p}_{n}}{\prod_{i=1}^{9}<p_{\ell_{i}}>^{2 d}} \prod_{j=0}^{n-1} \frac{1}{<p_{i_{j}}-p_{i_{j+1}}>^{2 d}} .
$$

for any $\ell_{1}, \ell_{2}, \ldots \ell_{9}$. Moreover, we also have the simple inequality

$$
\sup _{q} \frac{1}{<p-q>^{20 d}<q-r>^{20 d}} \leq \frac{C}{<p-r>^{20 d}} .
$$




\subsection{K-identity and Estimate.}

Lemma 3.1 . We have the following identity and estimate for $\eta>0$

$$
\begin{gathered}
K(t, \mathbf{p}, I)=i e^{t \eta / 2} \int d \alpha e^{-i \alpha t} \prod_{j \in I} \frac{1}{\alpha-p_{j}^{2} / 2+i \eta}, \\
|K(t, \mathbf{p}, I)| \leq C \int d \alpha \prod_{j \in I} \frac{1}{\left|\alpha-p_{j}^{2} / 2+i t^{-1}\right|}
\end{gathered}
$$

(note that the estimate is finite only for $|I| \geq 2$ ), and for any $a<1,|I| \geq 2$,

$$
\int|K(t, \mathbf{p}, I)|^{2} d \mu\left(\mathbf{p}_{I}\right) \leq \frac{\left(C_{a} t\right)^{|I|-1}}{[(|I|-1) !]^{a}} .
$$

Proof of Lemma 3.1. Since $s_{j} \geq 0$ and $\delta\left(t-\sum_{j \in I} s_{j}\right)$ automatically guarantee that all $s_{j} \leq t$, the upper integration bound of $t$ in the definition of $K(t, \mathbf{p}, I)(2.4)$ can be replaced with $\infty$. Hence

$$
\begin{aligned}
K(t, \mathbf{p}, I)= & (-i)^{|I|-1} e^{t \eta / 2} \int_{0}^{\infty} \cdots \int_{0}^{\infty}\left(\prod_{j \in I} d s_{j}\right) \prod_{j \in I} e^{-i s_{j}\left(p_{j}^{2} / 2-i \eta\right)} \delta\left(t-\sum_{j \in I} s_{j}\right) \\
= & (-i)^{|I|-1} e^{t \eta / 2} \int_{-\infty}^{\infty} d \alpha e^{-i \alpha t} \prod_{j \in I}\left(\int_{0}^{\infty} e^{i s_{j}\left(\alpha-\left(p_{j}^{2} / 2-i \eta\right)\right)} d s_{j}\right) \\
& =(-i)(-1)^{|I|-1} \int_{-\infty}^{\infty} d \alpha e^{-i \alpha t+t \eta / 2} \prod_{j \in I} \frac{-1}{\alpha-p_{j}^{2} / 2+i \eta} .
\end{aligned}
$$

Note that the same identity (and its proof) holds if we replace some of the $p_{j}^{2}$ in $K(t, \mathbf{p}, I)$ with $p_{j}^{2}-i \mu$ (here $\left.\mu \geq 0\right)$. Recall that $K(t, \mathbf{p}, I)$ depends only on the square of the momenta, i.e., the function

$$
K^{*}\left(t, \mathbf{p}^{2}, I\right):=K(t, \mathbf{p}, I)
$$

is well defined. The same proof yields the identity

$$
K^{*}(t, \mathbf{a}, I)=i e^{t \eta / 2} \int_{-\infty}^{\infty} d \alpha e^{-i \alpha t} \prod_{j \in I} \frac{1}{\alpha-a_{j} / 2+i \eta}
$$

for any $\mathbf{a}_{I}=\left\{a_{j}: j \in I\right\}$ with $\operatorname{Im} a \leq 0$. This remark will be needed in Lemma 5.5. The proof of the estimate (3.8) is trivial with the choice of $\eta:=t^{-1}$. In the following proof of (3.9), we shall take $I=\{0, \cdots, n\}$ for simplicity. 
We claim the following two estimates hold for $K$ with $n:=|I|-1$;

$$
\begin{gathered}
|K(t, \mathbf{p}, I)| \leq \frac{t^{n}}{n !}, \\
\int|K(t, \mathbf{p}, I)|^{2-a} d \mu\left(\mathbf{p}_{I}\right) \leq\left(C_{a} t^{1-a}\right)^{n} \quad 0<a<1, \quad n \geq 1 .
\end{gathered}
$$

The bound (3.9) follows from these two inequalities:

$$
\int|K(t, \mathbf{p}, I)|^{2} d \mu\left(\mathbf{p}_{I}\right) \leq\left(\frac{t^{n}}{n !}\right)^{a} \int|K(t, \mathbf{p}, I)|^{2-a} d \mu\left(\mathbf{p}_{I}\right) \leq \frac{\left(C_{a} t\right)^{n}}{[n !]^{a}} .
$$

The proof of (3.12) is trivial from the original definition of $K(2.4)$ with multiple time integrals. The denominator $n$ ! comes from the restriction $\sum_{j} s_{j}=t$ in the time integration. We now prove (3.13). From the indentity of $K(3.7)$

$$
\int|K(t, \mathbf{p}, I)|^{2-a} d \mu\left(\mathbf{p}_{I}\right) \leq C \int d \mu\left(\mathbf{p}_{I}\right)\left(\int_{-\infty}^{\infty} d \alpha \prod_{j \in I} \frac{1}{\left|\alpha-p_{j}^{2} / 2+i t^{-1}\right|}\right)^{2-a} .
$$

Denote by

$$
G\left(\alpha, p_{0}, p_{1}, \cdots, p_{n-2}\right):=\prod_{j \in I \backslash\{n-1, n\}} \frac{1}{\left|\alpha-p_{j}^{2} / 2+i t^{-1}\right|} .
$$

For any fixed $p_{n-1}, p_{n}$ denote by $\nu$ the probability measure

$$
\nu(d \alpha):=R^{-1}\left(\alpha, p_{n-1}, p_{n}\right) d \alpha
$$

where

$$
R\left(\alpha, p_{n-1}, p_{n}\right):=Z\left(p_{n-1}, p_{n}\right)\left|\alpha-A+i t^{-1}\right|\left|\alpha-B+i t^{-1}\right|, \quad A:=p_{n-1}^{2} / 2, B:=p_{n}^{2} / 2
$$

and

$$
Z\left(p_{n-1}, p_{n}\right):=\int_{-\infty}^{\infty} \frac{d \alpha}{|\alpha-A+i \eta||\alpha-B+i \eta|}, \quad \eta=t^{-1} .
$$

We have the simple estimate for $Z$ :

$$
Z\left(p_{n-1}, p_{n}\right) \leq \frac{C}{|A-B+i \eta|}\left[1+\log _{+}\left|\frac{A-B}{\eta}\right|\right]
$$


Rewrite the $d \alpha$ integration in (3.14) as

$$
\int_{-\infty}^{\infty} d \alpha \prod_{j \in I} \frac{1}{\left|\alpha-p_{j}^{2} / 2+i t^{-1}\right|}=Z\left(p_{n-1}, p_{n}\right) \int_{-\infty}^{\infty} \nu(d \alpha) G\left(\alpha, p_{0}, \cdots, p_{n-2}\right) .
$$

From the Hölder inequality,

$$
\begin{gathered}
\int|K(t, \mathbf{p}, I)|^{2-a} d \mu\left(\mathbf{p}_{I}\right)=\int d \mu\left(\mathbf{p}_{I}\right)\left[Z\left(p_{n-1}, p_{n}\right) \int_{-\infty}^{\infty} \nu(d \alpha) G\left(\alpha, p_{0}, \cdots, p_{n-2}\right)\right]^{2-a} \\
\leq \int d \mu\left(\mathbf{p}_{I}\right) Z\left(p_{n-1}, p_{n}\right)^{2-a} \int_{-\infty}^{\infty} \nu(d \alpha) G\left(\alpha, p_{0}, \cdots, p_{n-2}\right)^{2-a}
\end{gathered}
$$

Fix $p_{n-1}$ and $p_{n}$. Using (3.2), the $p_{0} \cdots, p_{n-2}$ integrations are explicitly estimated by

$$
\begin{aligned}
& \int d \mu\left(\mathbf{p}_{I}\right) Z\left(p_{n-1}, p_{n}\right)^{2-a} G\left(\alpha, p_{0}, \cdots, p_{n-2}\right)^{2-a} \\
& \leq \int \frac{d p_{n-1} d p_{n} Z\left(p_{n-1}, p_{n}\right)^{2-a}\left|\Phi\left(p_{n}\right)\right|^{2}}{<p_{n-1}>^{2 d}<p_{n}>^{2 d}} \int \frac{d p_{n-2}}{\left|\alpha-p_{n-2}^{2} / 2+i t^{-1}\right|^{2-a}<p_{n-2}-p_{n-1}>^{2 d}} \\
& \times \int \frac{d p_{n-3}}{\left|\alpha-p_{n-3}^{2} / 2+i t^{-1}\right|^{2-a}<p_{n-3}-p_{n-2}>^{2 d}} \times \cdots \times \int \frac{d p_{0}}{\left|\alpha-p_{0}^{2} / 2+i t^{-1}\right|^{2-a}<p_{0}-p_{1}>^{2 d}}
\end{aligned}
$$

The following inequality can be checked easily :

$$
\sup _{\alpha} \sup _{p_{j}} \int \frac{d p_{j-1}}{\left|\alpha-p_{j-1}^{2} / 2+i t^{-1}\right|^{2-a}<p_{j-1}-p_{j}>^{2 d}} \leq C_{a} t^{1-a} .
$$

From this inequality, the integrations of $p_{0} \cdots p_{n-2}$ (in this order) can be estimated by a constant times $t^{1-a}$. Hence from (3.21)

$$
\int|K(t, \mathbf{p}, I)|^{2-a} d \mu\left(\mathbf{p}_{I}\right) \leq\left(C_{a} t^{1-a}\right)^{n-1} \int \frac{\left|\Phi\left(p_{n}\right)\right|^{2} d p_{n-1} d p_{n}}{<p_{n-1}>^{2 d}<p_{n}>^{2 d}} Z\left(p_{n-1}, p_{n}\right)^{2-a} \int_{-\infty}^{\infty} \nu(d \alpha) .
$$

Since $\nu$ is a probability measure, $\int \nu(d \alpha)=1$. By the $Z$ estimate (3.19),

$$
\begin{aligned}
& \int|K(t, \mathbf{p}, I)|^{2-a} d \mu\left(\mathbf{p}_{I}\right) \\
& \quad \leq\left(C_{a} t^{1-a}\right)^{n-1} \int d p_{n-1} d p_{n} \frac{\left|\Phi\left(p_{n}\right)\right|^{2}\left(1+\log _{+}\left|2 t\left(p_{n-1}^{2}-p_{n}^{2}\right)\right|\right)^{2-a}}{<p_{n-1}>^{2 d}<p_{n}>^{2 d}\left|\left(p_{n-1}^{2}-p_{n}^{2}\right) / 2+i t^{-1}\right|^{2-a}} .
\end{aligned}
$$

We can now integrate $d p_{n-1}$ :

$$
\sup _{p_{n}} \int d p_{n-1} \frac{\left(1+\log _{+}\left|2 t\left(p_{n-1}^{2}-p_{n}^{2}\right)\right|\right)^{2-a}}{<p_{n-1}>^{2 d}<p_{n}>^{2 d}\left|\left(p_{n-1}^{2}-p_{n}^{2}\right) / 2+i t^{-1}\right|^{2-a}} \leq C_{a} t^{1-a} .
$$


Since $\Phi \in L^{2}$, the last integral in $(3.24)$ is bounded by $C_{a} t^{1-a}$. We have thus proved (3.13).

The proof of (3.13) has the following simple extension. Denote $\mathbf{k}=\left(k_{0}, k_{1}, \ldots k_{m}\right)$ and $\|\mathbf{k}\|=\sum k_{j}$. Define $Q^{*}$ (the usage of the star in the notation will be clear later on)

$$
Q^{*}(t ; \mathbf{p} ; \mathbf{k}):=K(t ; \underbrace{p_{0}, p_{0}, \ldots p_{0}}_{k_{0}+1}, \ldots, \underbrace{p_{m}, p_{m} \ldots, p_{m}}_{k_{m}+1}) .
$$

Then $\left|Q^{*}(t ; \mathbf{p} ; \mathbf{k})\right| \leq C \Sigma(t, \mathbf{p}, \mathbf{k})$ with

$$
\Sigma(t, \mathbf{p}, \mathbf{k}):=\int_{-\infty}^{\infty} d \alpha \prod_{j=0}^{m}\left|\frac{1}{\alpha-p_{j}^{2} / 2+i t^{-1}}\right|^{k_{j}+1} .
$$

Lemma 3.2 . For any $m \geq 1,0 \leq a<1$, we have

$$
\int\left|Q^{*}(t ; \mathbf{p}, \mathbf{k})\right|^{2} d \mu\left(\mathbf{p}_{m}\right) \leq \frac{\left(C_{a} t\right)^{m+2\|\mathbf{k}\|}}{[(m+\|\mathbf{k}\|) !]^{a}}
$$

and

$$
\int(\Sigma(t, \mathbf{p}, \mathbf{k}))^{2-a} d \mu\left(\mathbf{p}_{m}\right) \leq\left(C_{a} t\right)^{(1-a) m+(2-a)\|\mathbf{k}\|} .
$$

Proof of Lemma 3.2. Similar to the proof of (3.9), (3.27) follows from (3.28) and the simple bound

$$
\left|Q^{*}(t ; \mathbf{p}, \mathbf{k})\right| \leq \frac{t^{m+\|\mathbf{k}\|}}{(m+\|\mathbf{k}\|) !} .
$$

The proof of (3.28) is also similar to that of (3.13). Define $G=G_{1} G_{2}$ where

$G_{1}=\left|\alpha-p_{m-1}^{2} / 2+i t^{-1}\right|^{-k_{m-1}}\left|\alpha-p_{m}^{2} / 2+i t^{-1}\right|^{-k_{m}}, \quad G_{2}=\prod_{j=0}^{m-2}\left|\alpha-p_{j}^{2} / 2+i t^{-1}\right|^{-\left(k_{j}+1\right)}$.

We shall use the measure $\nu, R\left(\alpha, p_{m-1}, p_{m}\right)$ and $Z\left(p_{m-1}, p_{m}\right)$ as in the proof of the previous lemma. Then we can rewrite the left hand side of $(3.28)$ as $\int d \mu\left(\mathbf{p}_{m}\right)\left[\int \nu(d \alpha) Z G\right]^{2-a}$. We then use the Hölder inequality to arrive at

$$
\begin{aligned}
& \int|\Sigma(t, \mathbf{p}, \mathbf{k})|^{2-a} d \mu\left(\mathbf{p}_{m}\right) \\
& \leq \int d \mu\left(\mathbf{p}_{m}\right) Z\left(p_{m-1}, p_{m}\right)^{2-a} \int_{-\infty}^{\infty} \nu(d \alpha) G_{1}\left(\alpha, p_{m-1}, p_{m}\right)^{2-a} G_{2}\left(\alpha, p_{0}, \cdots, p_{m-2}\right)^{2-a}
\end{aligned}
$$


The integrations of $p_{0} \cdots p_{m-2}$ in $\int d \mu\left(\mathbf{p}_{m}\right) G_{2}^{2-a}$ can be estimated the same way if we use

$$
\sup _{\alpha} \sup _{p_{j}} \int \frac{d p_{j-1}}{\left|\alpha-p_{j-1}^{2} / 2+i t^{-1}\right|^{(k+1)(2-a)}<p_{j-1}-p_{j}>^{2 d}} \leq C_{a} t^{(1-a)+k(2-a)}
$$

We have thus proved that (cf. (3.23)):

$$
\begin{aligned}
\int d \mu\left(\mathbf{p}_{m}\right) & (\Sigma(t, \mathbf{p}, \mathbf{k}))^{2-a} \leq C_{a} t^{(m-1)(1-a)+\|k\|(2-a)-k_{m-1}(2-a)-k_{m}(2-a)} \\
& \times \int \frac{d p_{m-1} d p_{m}\left|\Phi\left(p_{m}\right)\right|^{2} Z\left(p_{m-1}, p_{m}\right)^{2-a}}{<p_{m-1}>^{2 d}<p_{m}>^{2 d}} \int \nu(d \alpha) G_{1}\left(\alpha, p_{m-1}, p_{m}\right)^{2-a}
\end{aligned}
$$

From the definition of $\nu$ and the $Z$ estimate (3.19), the last integration is bounded by

$$
\begin{aligned}
& \leq \int d \alpha \int d p_{m-1} d p_{m} \frac{\left|\Phi\left(p_{m}\right)\right|^{2}\left(1+\log _{+}\left|2 t\left(p_{m-1}^{2}-p_{m}^{2}\right)\right|\right)^{1-a}}{<p_{m-1}>^{2 d}<p_{m}>^{2 d}\left|\left(p_{m-1}^{2}-p_{m}^{2}\right) / 2+i t^{-1}\right| 1-a} \\
& \quad \times\left|\alpha-p_{m-1}^{2} / 2+i t^{-1}\right|^{-k_{m-1}(2-a)-1}\left|\alpha-p_{m}^{2} / 2+i t^{-1}\right|^{-k_{m}(2-a)-1}
\end{aligned}
$$

We now perform the $p_{m-1}$ integration. It is easy to check that it is bounded by $C_{a} t^{(1-a)+k_{m-1}(2-a)}$ uniformly for any values of $\alpha$ and $p_{m}$. Then we perform the $\alpha$ integration, which is bounded by $C_{a} t^{k_{m}(2-a)}$. If $k_{m}=0$, we have to borrow an extra decay in $\alpha$ from the $p_{m-1}$ cutoff to make the $\alpha$ integration finite. Finally, we use $\Phi \in L^{2}$ for the $p_{m}$ integration. Putting all these bounds together, we have proved the Lemma.

\section{$3.2 L^{2}$ Estimates.}

The key estimate is the following Lemma.

Lemma 3.3 . Recall the definition of $\psi_{n}$ in (2.6). We have for any $n$

$$
\mathbf{E}\left\|\psi_{n}(t)\right\|^{2} \leq\left(C \lambda^{2} t\right)^{n} \frac{1}{(n !)^{1 / 2}}\left[1+\frac{(n !)^{3 / 2}(\log t)^{n+3}}{t^{1 / 2}}\right] .
$$

Recall that $\lambda=\sqrt{\varepsilon}, t=T / \varepsilon$ and $T$ is fixed. Let $n_{0}=n_{0}(\varepsilon)$ be given in (2.2) with some $0<\gamma \ll 1$. It follows (cf.(2.3)) that

$$
\lim _{M \rightarrow \infty} \lim _{\varepsilon \rightarrow 0} \mathbf{E}\left\|\sum_{n=M}^{n_{0}(\varepsilon)-1} \psi_{n}\left(T \varepsilon^{-1}\right)\right\|^{2}=0 .
$$

Proof of Lemma 3.3. By definition of $\psi_{n}$ and $C_{\pi}:=C_{\pi}^{\delta(\xi)}$ defined in (2.17), we have

$$
\mathbf{E}\left\|\psi_{n}(t)\right\|^{2}=U_{n, n}=\sum_{\pi \in \Pi_{n, n}} C_{\pi}
$$

We shall estimate the contributions from crossing, nested and simple pairings separately. The proof of Lemma 3.3 follows from Lemmas 3.5, 3.6 and 3.7.

First we prove a weaker estimate: 
Lemma 3.4 . For any $\pi \in \Pi_{n, n}$

$$
\left|C_{\pi}\right| \leq\left(C \lambda^{2} t\right)^{n}(\log t)^{n+2}
$$

It follows immediately that

$$
\mathbf{E}\left\|\psi_{n}(t)\right\|^{2} \leq n !\left(C \lambda^{2} t\right)^{n}(\log t)^{n+2}
$$

Proof of Lemma 3.4. Since the number of pairings is $C^{n} n$ !, the second bound follows from the first one and (3.30). Recall the convention of relabelling $p$ in (2.18). We can now use the definition of $C_{\pi}(2.17)$ to have

$$
\left|C_{\pi}\right| \leq \lambda^{2 n} \int d \mathbf{w}_{2 n+1}|K(t, \mathbf{p}, n)|\left|K\left(t, \mathbf{p}^{\prime}, n\right)\right| \Delta_{\pi}^{0}\left(\mathbf{p}, \mathbf{p}^{\prime}\right)|F(\mathbf{p}, n)|\left|F\left(\mathbf{p}^{\prime}, n\right)\right|
$$

Define $\alpha_{\ell}:=\alpha$ if $\ell \leq n$ and $\alpha_{\ell}:=\beta$ otherwise. From the equation (2.19), we can solve the momentum $w_{j}$ for $j \in B$ as a function of $\mathbf{w}_{A}$. Together with the estimate of $K$ in (3.8) we obtain

$$
\left|C_{\pi}\right| \leq C \lambda^{2 n} \int d \mathbf{w}_{A} \int_{-\infty}^{\infty} d \alpha d \beta \prod_{0 \leq k \leq 2 n} \frac{1}{\left|\alpha_{k}-w_{k}^{2} / 2+i / t\right|}|F(\mathbf{p}, n)|\left|F\left(\mathbf{p}^{\prime}, n\right)\right| .
$$

Recall that the elements of $A$ are denoted by $a_{1}=0<a_{2}<\ldots<a_{\bar{n}+1}$ and here $\bar{n}=n$. From the definition of $F$ in (2.13), (3.2) and (3.6), we have

$$
|F(\mathbf{p}, n)|\left|F\left(\mathbf{p}^{\prime}, n\right)\right| \leq \frac{C^{n}\left|\Phi\left(w_{0}\right)\right|\left|\Phi\left(w_{2 \bar{n}+1}\right)\right|}{<w_{0}>^{2 d}<w_{k_{1}}>^{2 d}<w_{k_{2}}>^{2 d}} \prod_{\ell=2}^{n+1} \frac{1}{<w_{a_{\ell}}-w_{a_{\ell-1}}>^{2 d}}
$$

for any fixed $k_{1} \neq k_{2}$ and $k_{1}, k_{2}>0$. Recall that $p_{n}=w_{0}, p_{n}^{\prime}=w_{2 \bar{n}+1}, 0 \in A, 2 \bar{n}+1 \in B$. Recall that $\Delta_{\pi}^{0}$ always forces the delta function $\delta\left(w_{0}-w_{2 \bar{n}+1}\right)$. Thus we obtain for any $k>0$,

$$
\begin{aligned}
\left|C_{\pi}\right| \leq & \left(C \lambda^{2}\right)^{n} \int_{-\infty}^{\infty} d \alpha d \beta \int \frac{\left|\Phi\left(w_{0}\right)\right|^{2} d \mathbf{w}_{A}}{<w_{0}>^{2 d}<w_{k_{1}}>^{2 d}<w_{k_{2}}>^{2 d}} \frac{1}{\left|\alpha-w_{0}^{2} / 2+i / t\right|\left|\beta-w_{0}^{2} / 2+i / t\right|} \\
& \times \prod_{\ell=2}^{n+1} \frac{1}{\left|\alpha_{a_{\ell}}-w_{a_{\ell}}^{2} / 2+i / t\right|} \prod_{\ell=2}^{n+1} \frac{1}{<w_{a_{\ell}}-w_{a_{\ell-1}}>^{2 d}} \prod_{\substack{j \in B \\
j \neq 2 \bar{n}+1}} \frac{1}{\left|\alpha_{j}-w_{j}^{2} / 2+i / t\right|} .
\end{aligned}
$$

We choose $k_{1}, k_{2}$ such that $\alpha_{k_{1}}=\alpha$ and $\alpha_{k_{2}}=\beta$. It is easy to check that

$$
\sup _{w} \frac{1}{\left|\alpha_{j}-w^{2} / 2+i t^{-1}\right|<w>^{2 d}} \leq \frac{C t}{<\alpha_{j}>}
$$


We use this estimate for $w=w_{k_{2}}$. If we remove the factor $\langle w\rangle^{2 d}$ in the denominator, we only have to drop the $\left\langle\alpha_{j}\right\rangle$ in the denominator. Using this bound, we can estimate all the other factors depending on the momenta from $B$ in the expression (3.31). We now estimate integrations of momenta in $A$. We can check the simple inequality

$$
\sup _{w_{a_{\ell-1}}} \int \frac{d w_{a_{\ell}}}{<w_{a_{\ell}}>^{2 d}} \frac{1}{\left.\left|\alpha_{a_{\ell}}-w_{a_{\ell}}^{2} / 2+i / t\right|<w_{a_{\ell}}-w_{a_{\ell-1}}\right\rangle^{2 d}} \leq \frac{C \log t}{\left.<\alpha_{a_{\ell}}\right\rangle} .
$$

Again, if we remove the denominator $\left\langle w_{a_{\ell}}\right\rangle^{2 d}$, the denominator $\left\langle\alpha_{a_{\ell}}>\right.$ on the right hand side has to be removed. We can now apply the last estimate to integration for $A$-momenta except $w_{0}$. Hence

$$
\left|C_{\pi}\right| \leq\left(C \lambda^{2} t\right)^{n}(\log t)^{n} \int \frac{\left|\Phi\left(w_{0}\right)\right|^{2} d w_{0}}{<w_{0}>^{2 d}} \int_{-\infty}^{\infty} \frac{d \alpha d \beta}{<\alpha><\beta>\left|\alpha-w_{0}^{2} / 2+i / t\right|\left|\beta-w_{0}^{2} / 2+i / t\right|} .
$$

The denominator $\langle\alpha\rangle\langle\beta\rangle$ comes from the choice of $k_{1}, k_{2}$ and the denominators on the right hand side of (3.32), (3.33). Using

$$
\sup _{w} \int_{-\infty}^{\infty} \frac{d \alpha}{\langle\alpha\rangle\left|\alpha-w^{2} / 2+i / t\right|} \leq C \log t
$$

we can bound the last integration above by $(C \log t)^{2}$. Finally, by $\Phi \in L^{2}$, we obtain

$$
\left|C_{\pi}\right| \leq\left(C \lambda^{2} t\right)^{n}(\log t)^{n+2} .
$$

The following remark will be useful later. Suppose we consider only the product $\widetilde{\Delta}_{\pi}^{0}$ of a subset of delta functions from $\Delta_{\pi}^{0}$ with rank $r$ according to the setup of part (II) of Lemma 2.3. Then the method of the previous proof gives

$\int d \mathbf{w}_{2 n+1}|K(t, \mathbf{p}, n)|\left|K\left(t, \mathbf{p}^{\prime}, n\right)\right| \widetilde{\Delta}_{\pi}^{0}\left(\mathbf{p}, \mathbf{p}^{\prime}\right) G\left(\mathbf{p}, n ; \mathbf{p}^{\prime}, n\right)\left|\widehat{\psi}_{0}\left(p_{n}\right)\right|\left|\widehat{\psi}_{0}\left(p_{n}^{\prime}\right)\right| \leq(C t)^{r-1}(\log t)^{2 n-r+3}$ for any function $G$ with

$$
G\left(\mathbf{p}, n ; \mathbf{p}^{\prime}, n\right) \leq \prod_{i=0}^{2 n} \frac{1}{<w_{i}-w_{i+1}>^{20 d}} .
$$

Moreover, the numbers of momenta within the two $K$-kernels do not have to be the same. In full generality, the same proof as above gives

$$
\begin{aligned}
& \int d \mathbf{p}_{n} d \mathbf{p}_{n^{\prime}}^{\prime}|K(t, \mathbf{p}, n)|\left|K\left(t, \mathbf{p}^{\prime}, n^{\prime}\right)\right| \widetilde{\Delta}_{\pi}^{0}\left(\mathbf{p}_{n}, \mathbf{p}_{n^{\prime}}^{\prime}\right) G\left(\mathbf{p}, n ; \mathbf{p}^{\prime}, n^{\prime}\right)\left|\widehat{\psi}_{0}\left(p_{n}\right)\right|\left|\widehat{\psi}_{0}\left(p_{n^{\prime}}^{\prime}\right)\right| \\
& \leq(C t)^{r-1}(\log t)^{n+n^{\prime}-r+3}
\end{aligned}
$$

provided that $\widetilde{\Delta}_{\pi}^{0}$ can be written in the form of (2.19) with $|B|=r$ and $\delta\left(p_{n}-p_{n^{\prime}}^{\prime}\right)$ is forced by $\widetilde{\Delta}_{\pi}^{0}$. 


\subsection{Estimate of crossing pairings.}

Lemma 3.5 . If $\pi \in \Pi_{n, n}$ is crossing, then we have

$$
\left|C_{\pi}\right| \leq t^{-1}\left(C \lambda^{2} t\right)^{n}(\log t)^{n+3}
$$

Notice the extra factor $t^{-1}$ compared with the previous Lemma.

Proof of Lemma 3.5. First we present the proof for $d \geq 3$. The case $d=2$ requires some technical modicification which is discussed in Section 6. Since $\pi$ is crossing, for some $j_{0} \in B$ with $j_{0} \neq 2 \bar{n}+1$ we have

$$
w_{j_{0}}= \pm w_{\ell_{1}} \pm w_{\ell_{2}} \pm \ldots
$$

where $\ell_{1}, \ell_{2}, \ldots \in A$ according to Lemma 2.7. Notice that Lemma 2.3 implies that there are at least three momenta on the right hand side, hence we can assume that $\ell_{1}, \ell_{2} \neq 0$.

Similarly to $(3.31)$ we have (recall that the delta function $\delta\left(w_{0}-w_{2 \bar{n}+1}\right)$ is forced)

$$
\begin{aligned}
\left|C_{\pi}\right| \leq & \lambda^{2 n} \int_{-\infty}^{\infty} d \alpha d \beta \int d \mu\left(\mathbf{w}_{A}\right) \frac{1}{\left|\alpha-w_{0}^{2} / 2+i / t\right|\left|\beta-w_{0}^{2} / 2+i / t\right|} \\
& \times\left[\prod_{\substack{\ell \in A \\
\ell \neq 0}} \frac{1}{\left|\alpha_{\ell}-w_{\ell}^{2} / 2+i / t\right|}\right]\left|\frac{1}{\alpha_{j_{0}}-w_{j_{0}}^{2} / 2+i / t}\right|_{w_{j_{0}}=\left( \pm w_{\ell_{1}} \pm w_{\ell_{2}} \pm \ldots\right)} Y_{B}
\end{aligned}
$$

where

$$
Y_{B}=\prod_{\substack{j \in B \\ j \neq j_{0}, 2 \bar{n}+1}} \frac{1}{\left|\alpha_{j}-w_{j}^{2} / 2+i / t\right|}
$$

and

$$
d \mu\left(\mathbf{w}_{A}\right):=\frac{\left|\Phi\left(w_{0}\right)\right|^{2} d \mathbf{w}_{A}}{<w_{0}>^{20 d}} \prod_{j=1}^{n-1} \frac{1}{<w_{a_{j}}-w_{a_{j+1}}>^{20 d}}
$$

(notice that the argument of $\Phi$ is $w_{0}$ and not $w_{n}$ ). We can bound $\left|\alpha_{j}-w_{j}^{2} / 2+i / t\right|^{-1}$ by $t^{-1}$. Thus the last factor $Y_{B}$ is bounded by $t^{n-1}$. One can easily check the inequality for $d \geq 3$

$$
\begin{aligned}
\sup _{\gamma_{i}=\alpha, \beta} \sup _{v} \int d w_{1} \int d w_{2} & <w_{1}>^{-2 d}<w_{2}>^{-2 d}\left|\gamma_{0}-\left( \pm w_{1} \pm w_{2}+v\right)^{2} / 2+i / t\right|^{-1} \\
& \times \frac{1}{\left|\gamma_{1}-w_{1}^{2} / 2+i / t\right|} \frac{1}{\left|\gamma_{2}-w_{2}^{2} / 2+i / t\right|} \leq C(\log t)^{3} .
\end{aligned}
$$

We can use this inequality to estimate the $w_{\ell_{1}}$ and $w_{\ell_{2}}$ integrations. After this, the integrations of $\alpha$ and $\beta$ and the other momenta in (3.37) are now similar to the proof of the previous 
Lemma. First we integrate all momenta but $w_{0}$. We gain an extra factor $<\alpha>^{-1}<\beta>^{-1}$ (cf. (3.32), (3.33)), and we obtain

$$
\begin{aligned}
\left|C_{\pi}\right| \leq & \left(C \lambda^{2} t\right)^{n} t^{-1}(\log t)^{n+1} \int \frac{\left|\Phi\left(w_{0}\right)\right|^{2} d w_{0}}{<w_{0}>^{2 d}} \\
& \times \int_{-\infty}^{\infty} \frac{d \alpha d \beta}{<\alpha><\beta>\left|\alpha-w_{0}^{2} / 2+i / t\right|\left|\beta-w_{0}^{2} / 2+i / t\right|}
\end{aligned}
$$

Then we integrate $\alpha, \beta$ using (3.34). The last integration is $d w_{0}$ and we have to use $\Phi \in L^{2}$. Each integration gives at most a factor $\log t$. The details are left to the reader. Hence (3.37) is bounded by

$$
\left|C_{\pi}\right| \leq\left(C \lambda^{2} t\right)^{n} t^{-1}(\log t)^{n+3}
$$

which completes the proof for $d \geq 3$.

\subsection{Estimate of nested pairings.}

Lemma 3.6 . If $\pi \in \Pi_{n, n}$ is nested then

$$
\left|C_{\pi}\right| \leq\left(C \lambda^{2} t\right)^{n} t^{-1}(\log t)^{n+2}
$$

Proof of Lemma 3.6. A nested pairing (Fig. 2) contains two delta functions of the type $\delta\left(-p_{i}+p_{i+1}-p_{j}+p_{j+1}\right)$ and $\delta\left(-p_{k}+p_{k+1}-p_{u}+p_{u+1}\right)$ with $i<k<u<j<n$ (or the same type of delta functions with all primed variables. Without loss of generality we can assume that a nested pairing occurs in the nonprimed variables). Recall that this pair of delta functions is called a nest with an outer and an inner delta function (the first one is called outer, the second one is inner).

A nested pairing can have several inner delta function. Consider a nest which is minimal in the sense that none of its inner delta function is an outer delta function in some other nest. Since the pairing has no crossing, all the inner delta functions in this nest are joining two neighboring bullets. In other words, for all inner delta function $\delta\left(-p_{k}+p_{k+1}-p_{u}+p_{u+1}\right)$ we have $u=k+1$ (as in Fig. 2) and hence $p_{k}=p_{k+2}$ is forced. Let $p_{c_{1}}, p_{c_{2}}, \ldots p_{c_{v}}$ be those momenta with $i+1=c_{1}<c_{2}<\ldots<c_{v}=j$ which are forced to be the same as $p_{k}=p_{k+2}$, i.e.

$$
p_{c_{1}}=p_{c_{2}}=\ldots=p_{k}=p_{k+2}=\ldots=p_{c_{v}}
$$


(in particular $k$ and $k+2$ are among the $c_{1}, c_{2}, \ldots c_{v}$, i.e. $v \geq 2$ ). By Lemma 2.6, these are those momentum lines in the graph among $p_{i+1}, p_{i+2}, \ldots p_{j}$ such that if we remove any of them along with $p_{k}$, the graph becomes disconnected (and we include $p_{k}$ as well). On Fig. 2 $v=4, c_{1}=i+1, c_{2}=k, c_{3}=k+2, c_{4}=j$.

It is obvious that $\Delta_{\pi}^{0}$ does not depend on $p_{k+1}$. Since the only delta function that contains $p_{k+1}$ is $\delta\left(-p_{k}+p_{k+1}-p_{k+1}+p_{k+2}\right)$. We also claim that using the notations (3.41)

$$
\Delta_{\pi}^{0}(\mathbf{w})=\widetilde{\Delta}_{\pi}^{0}\left(\mathbf{p}^{*}, \mathbf{p}^{\prime}\right) \times \prod_{a=1}^{v-1} \delta\left(p_{c_{a}}-p_{c_{a+1}}\right)
$$

where $\mathbf{p}^{*}:=\mathbf{p}_{I^{*}}$ with

$$
I^{*}:=I_{n} \backslash\left\{c_{1}, c_{2}, \ldots c_{v}\right\}
$$

and $\widetilde{\Delta}_{\pi}^{0}\left(\mathbf{p}^{*}, \mathbf{p}^{\prime}\right)$ is a product of delta functions depending only on $\mathbf{p}^{*}, \mathbf{p}^{\prime}$. The point is that the momenta $\left\{p_{c_{1}}, p_{c_{2}}, \ldots\right\}$ occur only in the identifying delta functions as explicitly written in (3.42). The decomposition (3.42) is obvious from the graph using Lemma 2.6. [ The formal proof goes as follows. Since $\pi$ is noncrossing, we know from Lemma 2.7 that $\Delta_{\pi}^{0}$ has a representation where all delta functions are identifications. Consider this representation. If a momentum $p_{c_{a}}(1 \leq a \leq v)$ appears in a delta function, then it has the form $\delta\left(p_{c_{a}}-w_{r}\right)$ or $\delta\left(w_{r}-p_{c_{a}}\right)$ for some $r$. We claim that $r=c_{b}$ with some $1 \leq b \leq v$, i.e. $p_{c_{a}}$ appears only in the product of delta functions identifying $p_{c_{1}}=p_{c_{2}}=\ldots=p_{c_{v}}$. Clearly $i<r \leq j$, otherwise the outer delta function would prevent the graph becoming disconnected if we remove the edge $e\left(w_{r}\right)$ and $e\left(p_{c_{a}}\right)$. But then $r$ must be one of the $c_{b}$ as $\Delta_{\pi}^{0}$ does not depend on other momenta with index between $i$ and $j$ by the minimality of the nest. ]

Remark that $\widetilde{\Delta}_{\pi}^{0}\left(\mathbf{p}^{*}, \mathbf{p}^{\prime}\right)$ forces $p_{n}=p_{n}^{\prime}$. For, (3.42) decomposes $\Delta_{\pi}^{0}(\mathbf{w})$ into two factors having no common variables. Since $p_{n}, p_{n}^{\prime}$ belong to the arguments of $\widetilde{\Delta}_{\pi}^{0}\left(\mathbf{p}^{*}, \mathbf{p}^{\prime}\right)$, and $\Delta_{\pi}^{0}(\mathbf{w})$ forces $p_{n}=p_{n}^{\prime}$, hence $\widetilde{\Delta}_{\pi}^{0}\left(\mathbf{p}^{*}, \mathbf{p}^{\prime}\right)$ does so, too.

We also need the following identity

$$
K(t, \mathbf{p}_{I}, \underbrace{q, q, \ldots q}_{v \text { times }})=(-i)^{v} \int_{0}^{t} d s \frac{s^{v-1}}{(v-1) !} e^{-i s q^{2} / 2} K(t-s, \mathbf{p}, I) .
$$

Recall the definition

$$
C_{\pi}=\lambda^{2 n} \int d \mathbf{p}_{n} d \mathbf{p}_{n}^{\prime} \bar{K}(t, \mathbf{p}, n) K\left(t, \mathbf{p}^{\prime}, n\right) \Delta_{\pi}^{0}\left(\mathbf{p}, \mathbf{p}^{\prime}\right) \overline{F(\mathbf{p}, n)} F\left(\mathbf{p}^{\prime}, n\right) .
$$


Define the function

$$
E\left(\mathbf{p}^{*}, \mathbf{p}_{n}^{\prime}, p_{k}\right):=\left[\overline{F(\mathbf{p}, n)} F\left(\mathbf{p}^{\prime}, n\right)\right]_{p_{c_{a}}=p_{k}, a=1 \cdots, v},
$$

which inherits the smoothness in $p_{k}$ and decay from $F$. Together with the identity (3.43) and the notation of $\mathbf{p}^{*}$, we have

$C_{\pi}=\lambda^{2 n} \int d \mathbf{p}^{*} d p_{k} d \mathbf{p}_{n}^{\prime} \int_{0}^{t} d s \frac{s^{v-1} i^{v}}{(v-1) !} e^{i s p_{k}^{2} / 2} \overline{K\left(t-s, \mathbf{p}, I^{*}\right)} K\left(t ; \mathbf{p}^{\prime}, n\right) \widetilde{\Delta}_{\pi}^{0}\left(\mathbf{p}^{*}, \mathbf{p}^{\prime}\right) E\left(\mathbf{p}^{*}, \mathbf{p}_{n}^{\prime}, p_{k}\right)$.

We can integrate $p_{k}$ by parts. We first rewrite the $d$-dimensional integration as

$\int s^{v-1} e^{i s p_{k}^{2} / 2} E\left(\mathbf{p}^{*}, \mathbf{p}_{n}^{\prime}, p_{k}\right) d p_{k}=(2 \pi)^{-d / 2} \int_{0}^{\infty} s^{v-1} e^{i s Q / 2}\left(\int_{p_{k}^{2}=Q} E\left(\mathbf{p}^{*}, \mathbf{p}_{n}^{\prime}, p_{k}\right) d \omega\right) Q^{\frac{d}{2}-1} d Q$,

where $d \omega$ is the normalized surface measure on the sphere $p_{k}^{2}=Q$ in the $p_{k}$ space. Define

$$
\begin{gathered}
H\left(\mathbf{p}^{*}, \mathbf{p}_{n}^{\prime}, p_{k}\right):=2 i Q^{-\frac{d}{2}+1} \frac{\partial}{\partial Q}\left(Q^{\frac{d}{2}-1} \int_{p_{k}^{2}=Q} E\left(\mathbf{p}^{*}, \mathbf{p}_{n}^{\prime}, p_{k}\right) d \omega\right)_{Q:=p_{k}^{2}} \\
=i(d-2) p_{k}^{-2} \int E\left(\mathbf{p}^{*}, \mathbf{p}_{n}^{\prime}, p_{k}\right) d \omega+2 i \frac{\partial}{\partial Q}\left(\int_{p_{k}^{2}=Q} E\left(\mathbf{p}^{*}, \mathbf{p}_{n}^{\prime}, p_{k}\right) d \omega\right)_{Q:=p_{k}^{2}} .
\end{gathered}
$$

We can integrate $Q$ by parts to have for $v \geq 2$,

$$
\int s^{v-1} e^{i s p_{k}^{2} / 2} E\left(\mathbf{p}^{*}, \mathbf{p}_{n}^{\prime}, p_{k}\right) d p_{k}=\int s^{v-2} e^{i s p_{k}^{2} / 2} H\left(\mathbf{p}^{*}, \mathbf{p}_{n}^{\prime}, p_{k}\right) d p_{k} .
$$

Notice that $H$ inherits the decay from $F$. There is a singularity at $p_{k}=0$, integrable for dimension $d \geq 3$. Notice this singular term disappears in dimension $d=2$.

We can now rewrite $C_{\pi}$ as $C_{\pi}=\frac{\lambda^{2 n} i^{v}}{v-1} \int d \mathbf{p}^{*} d p_{k} d \mathbf{p}_{n}^{\prime} \int_{0}^{t} d s \frac{s^{v-2}}{(v-2) !} e^{i s p_{k}^{2} / 2} \overline{K\left(t-s, \mathbf{p}, I^{*}\right)} K\left(t, \mathbf{p}^{\prime}, n\right) \widetilde{\Delta}_{\pi}^{0}\left(\mathbf{p}^{*}, \mathbf{p}^{\prime}\right) H\left(\mathbf{p}^{*}, \mathbf{p}_{n}^{\prime}, p_{k}\right)$.

Using the identity (3.43) to integrate $s$, we have

$$
\begin{aligned}
C_{\pi}= & \frac{i \lambda^{2 n}}{v-1} \int d \mathbf{p}^{*} d p_{k} d \mathbf{p}_{n}^{\prime}\left(\prod_{a=1}^{v-2} d q_{a}\right) \overline{K\left(t, \mathbf{p}^{*}, p_{k}, q_{1}, q_{2}, \ldots q_{v-2}\right)} \\
& \times K\left(t, \mathbf{p}^{\prime}, n\right) \widetilde{\Delta}_{\pi}^{0}\left(\mathbf{p}^{*}, \mathbf{p}^{\prime}\right)\left(\prod_{a=1}^{v-2} \delta\left(p_{k}-q_{a}\right)\right) H\left(\mathbf{p}^{*}, \mathbf{p}_{n}^{\prime}, p_{k}\right) .
\end{aligned}
$$


where we have written

$$
\overline{K(t, \mathbf{p}^{*}, \underbrace{p_{k}, p_{k}, \ldots p_{k}}_{v-1 \text { times }})}=\left(\int \prod_{a=1}^{v-2} d q_{a}\right) \overline{K\left(t, \mathbf{p}^{*}, p_{k}, q_{1}, q_{2}, \ldots q_{v-2}\right)}\left(\prod_{a=1}^{v-2} \delta\left(p_{k}-q_{a}\right)\right) .
$$

Notice that we now have momenta $\mathbf{p}^{*}, p_{k}, q_{1}, q_{2}, \ldots q_{v-2}$ and $\mathbf{p}_{n}^{\prime}$. The number of momenta $\mathbf{p}_{n}^{\prime}$ is $n+1$; that of $\mathbf{p}^{*}, p_{k}, q_{1}, q_{2}, \ldots q_{v-2}$ is $n$. The new delta function in (3.47) is

$$
\widetilde{\Delta}_{\pi}^{0}\left(\mathbf{p}^{*}, \mathbf{p}^{\prime}\right)\left(\prod_{a=1}^{v-2} \delta\left(p_{k}-q_{a}\right)\right)
$$

which has rank $n$ and it still forces $p_{n}=p_{n}^{\prime}$. The function $H$ depends on $\mathbf{p}^{*}, p_{k}$ and $\mathbf{p}_{n}^{\prime}$. Define a function $H^{*}$ depending on $\mathbf{p}^{*}, p_{k}, q_{1}, q_{2}, \ldots q_{v-2}$ and $\mathbf{p}_{n}^{\prime}$ by

$$
H^{*}\left(\mathbf{p}^{*}, p_{k}, q_{1}, q_{2}, \ldots q_{v-2}, \mathbf{p}_{n}^{\prime}\right):=H\left(\mathbf{p}^{*}, \mathbf{p}_{n}^{\prime}, p_{k}\right) \prod_{a=1}^{v-2} \frac{1}{<p_{k}-q_{a}>^{20 d}} .
$$

Here we have included the cutoff in large $q_{a}$. Due to the delta function $\left(\prod_{a=1}^{v-2} \delta\left(p_{k}-q_{a}\right)\right)$, we can replace $H$ in (3.47) by $H^{*}$ without changing its value. We are now in the same setup as in Lemma 3.4 except that the rank of the delta functions (3.48) is now $n$, reduced from $n+1$. We can apply the remark at the end of the proof of Lemma 3.4, (3.35), to have

$$
\left|C_{\pi}\right| \leq\left(C \lambda^{2} t\right)^{n} t^{-1}(\log t)^{n+2} .
$$

\subsection{Estimate of simple pairings.}

Lemma 3.7 . If $\pi \in \Pi_{n, n^{\prime}}$ is simple, then

$$
\left|C_{\pi}\right| \leq \frac{\left(C \lambda^{2} t\right)^{\bar{n}}}{(\bar{n} !)^{1 / 2}}+O\left(\left(C_{a} \lambda^{2} t\right)^{\bar{n}} t^{-\frac{a}{2}}\right)
$$

for any $0 \leq a<1$ (recall that $\left.\bar{n}=\left(n+n^{\prime}\right) / 2\right)$.

Before the proof, we need several notations. The structure of the momenta in a simple pairing can easily be described. We shall rename the variables according to the pairing. Though for the $L^{2}$-norm we need to consider pairings $\pi \in \Pi_{n, n}$ only, we set up the formulas for the more general case $\pi \in \Pi_{n, n^{\prime}}$ needed later on for computing the Wigner transform.

First notice that a simple pairing has the property that all Type I delta functions are minimal, i.e., for any Type I delta function $\delta\left(-p_{i}+p_{i+1}-p_{j}+p_{j+1}\right)$ in a simple pairing we have $j=i+1$; and similarly for Type I'. Hence this delta function does not depend on $p_{j} \equiv p_{i+1}$. Moreover, since each momentum appears exactly twice in the delta functions, we see that $\Delta_{\pi}^{0}$ is independent of $p_{j} \equiv p_{i+1}$. 
Definition 3.8. In case of a simple pairing $\pi$, we call a momentum external if $\Delta_{\pi}^{0}$ depends on it. Otherwise it is called internal. We shall use the letter $r$ for external and $q$ for internal momenta.

Let $\underline{\mathbf{k}}$ be an array of double indices for the internal momenta

$$
\underline{\mathbf{k}}:=\left(01,02, \ldots 0 k_{0}, 11,12, \ldots 1 k_{1}, \ldots m k_{m}\right)
$$

where $\mathbf{k}=\left(k_{0}, k_{1}, \ldots k_{m}\right)$ and $k_{j} \geq 0$ will denote the number of the internal momenta for $j$. We shall denote $\|\mathbf{k}\|=\sum k_{j}$ and $|\mathbf{k}|:=m$. These numbers will depend on the structure of the pairing $\pi$. Let $|\pi|:=m$. Define

$$
\mathbf{r}_{m}=\left(r_{j}\right)_{0 \leq j \leq m}, \quad \mathbf{r}_{m, \widehat{0}}=\left(r_{j}\right)_{1 \leq j \leq m}
$$

to be the external momenta. Denote the internal momenta by $\underline{\mathbf{q}}=\left(q_{j \ell}\right)_{0 \leq \ell<\infty, 0 \leq j<\infty}$ and denote

$$
\underline{\mathbf{q}}_{\mathbf{k}}=\left(q_{j \ell}\right)_{1 \leq \ell \leq k_{j}, 0 \leq j \leq m} .
$$

The following lemma about the structure of a simple pairing (see also Fig 3.) will be used.

Lemma 3.9. If $\pi$ is simple, then it has $m=|\pi|$ Type II delta functions: $\delta\left(-p_{i_{j}-1}+p_{i_{j}}+\right.$ $\left.p_{i_{j}^{\prime}-1}^{\prime}-p_{i_{j}^{\prime}}^{\prime}\right)$ with $j=1,2, \ldots m$ and $i_{0}:=0<i_{1}<\ldots<i_{m}, i_{0}^{\prime}:=0<i_{1}^{\prime}<\ldots<i_{m}^{\prime}$. For each $j=0,1, \ldots, m$ there are $k_{j}$ Type I delta functions in the form $\delta\left(-p_{u}+p_{u+1}-p_{u+1}+p_{u+2}\right)=$ $\delta\left(-p_{u}+p_{u+2}\right)$ with $i_{j} \leq u<i_{j+1}$. We shall rename the momenta such that $r_{j}:=p_{i_{j}}$, and $q_{j \ell}:=p_{u+1}$ if $\delta\left(-p_{u}+p_{u+1}-p_{u+1}+p_{u+2}\right)=\delta\left(-p_{u}+p_{u+2}\right)$ is the $\ell^{\text {th }}$ Type I delta function with $i_{j} \leq u<i_{j+1}$. The notations are similar for the Type I' delta functions. We claim that $\Delta_{\pi}^{0}$ is equivalent to the product of the following delta functions

$$
\begin{gathered}
\delta\left(p_{i_{j}}-p_{i_{j}^{\prime}}^{\prime}\right) \quad j=0,1, \ldots, m \\
\delta\left(p_{i_{j}}-p_{u+2}\right)
\end{gathered}
$$

for each $u$ such that $q_{j \ell}=p_{u+1}$ for $\ell=1,2, \ldots, k_{j}, j=0,1, \ldots, m$ (i.e. $p_{u}$ and $p_{u+2}$ are the "external legs" belonging to the internal momentum $p_{u+1}$ ); and

$$
\delta\left(p_{i_{j}}-p_{u+2}^{\prime}\right)
$$


for each $u$ such that $q_{j \ell}^{\prime}=p_{u+1}^{\prime}, \ell=1,2, \ldots, k_{j}^{\prime}, j=0,1, \ldots, m$. The total number of potentials is

$$
2 \bar{n}=n+n^{\prime}=2\left(|\mathbf{k}|+\|\mathbf{k}\|+\left\|\mathbf{k}^{\prime}\right\|\right)
$$

and we also have the relation

$$
n=m+2\|\mathbf{k}\|, \quad n^{\prime}=m+2\left\|\mathbf{k}^{\prime}\right\| .
$$

The total number of delta functions in (3.50), (3.51), (3.52) is $m+1+\|\mathbf{k}\|+\left\|\mathbf{k}^{\prime}\right\|=\bar{n}+1$.

Proof of Lemma 3.9.

For simplicity, we redraw the graph of a simple pairing such that the pairing lines corresponding to Type II deltafunctions are straight lines inside $C$. These lines do not cross each other. An application of Lemma 2.6 shows that the delta functions (3.50), (3.51) and (3.52) are forced by $\Delta_{\pi}^{0}$. Each of these identifications contains at least one momentum which does not appear elsewhere, hence the rank of the product of these identifications is the same as the number of delta functions in (3.50), (3.51) and (3.52). Simple counting shows that these numbers are $m+1,\|\mathbf{k}\|$ and $\left\|\mathbf{k}^{\prime}\right\|$, respectively, hence the rank is $m+1+\|\mathbf{k}\|+\left\|\mathbf{k}^{\prime}\right\|=\bar{n}+1$ which equals to the rank of $\Delta_{\pi}^{0}$ by Lemma 2.3. This shows that $\Delta_{\pi}^{0}$ is equivalent to the product of (3.50), (3.51) and (3.52). For example, the simple pairing on Fig. 3 has $m=3$, $\mathbf{k}=(0,0,2,0), \mathbf{k}^{\prime}=(0,1,0,0), n=7, n^{\prime}=5$.

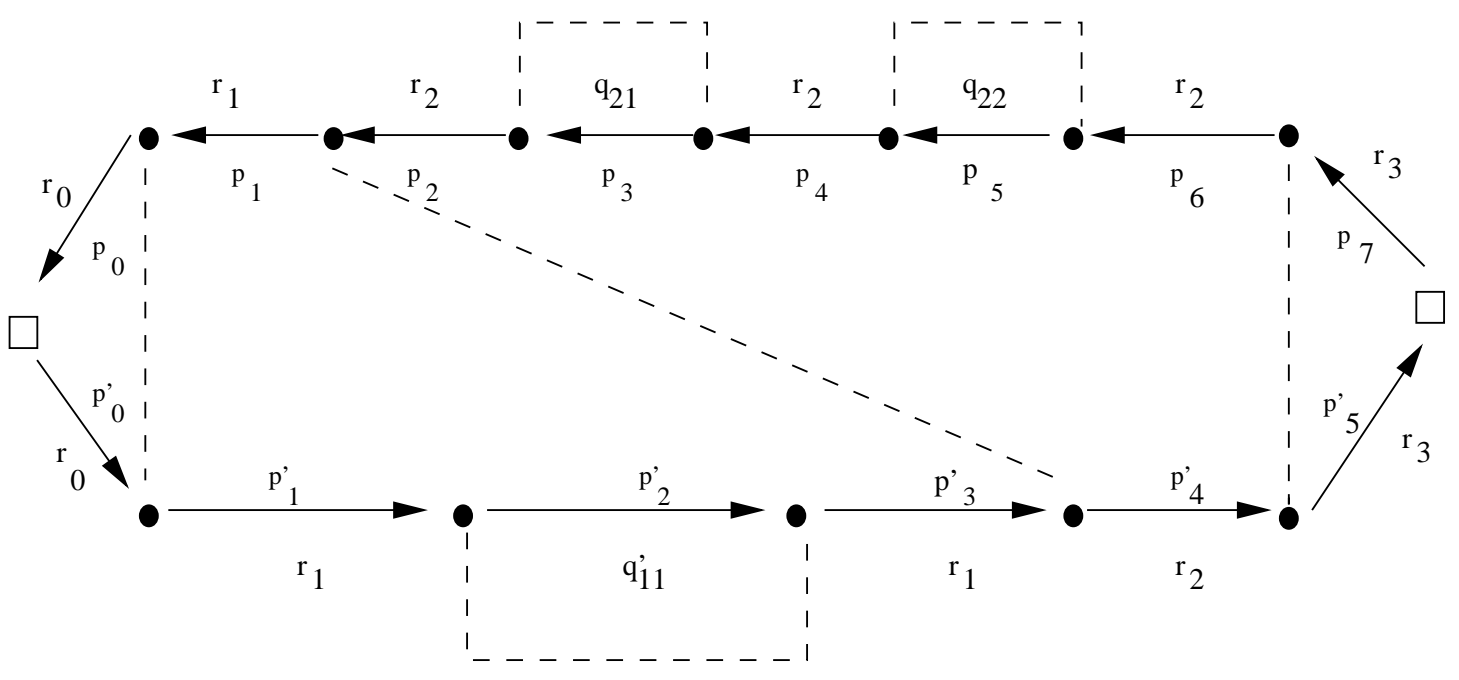

Fig. 3

We now introduce some more notations associated with the new labeling. Recall that for 
any set of momenta $\mathbf{p}_{I}$ the free evolution kernel is

$$
K(t ; \mathbf{p}, I):=(-i)^{|I|-1} \int_{0}^{t}\left(\prod_{j \in I} d s_{j}\right) \delta\left(t-\sum_{j \in I} s_{j}\right) \prod_{j \in I} e^{-i s_{j} p_{j}^{2} / 2} .
$$

For any disjoint sets $I, J$, we have the semigroup property

$$
K(t ; \mathbf{p}, I \cup J)=-i \int_{0}^{t} \int_{0}^{t} d s_{1} d s_{2} \delta\left(t-s_{1}-s_{2}\right) K\left(s_{1} ; \mathbf{p}, I\right) K\left(s_{2} ; \mathbf{p}, J\right) .
$$

Define for $\mathbf{k}=\left(k_{0}, k_{1}, \ldots k_{m}\right)$

$$
Q(t ; \mathbf{r}, \underline{\mathbf{q}} ; \mathbf{k}):=K(t ; \underbrace{r_{0}, r_{0}, \ldots r_{0}}_{k_{0}+1}, q_{01}, \ldots, q_{0 k_{0}}, \ldots, \underbrace{r_{m}, r_{m} \ldots, r_{m}}_{k_{m}+1}, q_{m 1}, \ldots, q_{m k_{m}}) .
$$

From the semigroup equality (3.53) we can rewrite $Q$ as

$$
Q(t ; \mathbf{r}, \underline{\mathbf{q}} ; \mathbf{k})=(-i)^{m} \int_{0}^{t}\left(\prod_{j=0}^{m} d s_{j}\right) \delta\left(t-\sum_{j=0}^{m} s_{j}\right) \prod_{j=0}^{m} K(s_{j} ; \underbrace{r_{j}, \ldots, r_{j}}_{k_{j}+1}, q_{j 1}, \cdots, q_{j k_{j}}) .
$$

Clearly, $Q(t ; \mathbf{r}, \underline{\mathbf{q}} ; \mathbf{k})=K(t, \mathbf{p}, n)$. If there is no internal momentum, we denote it by

$$
Q^{*}(t ; \mathbf{r} ; \mathbf{k}):=K(t ; \underbrace{r_{0}, r_{0}, \ldots r_{0}}_{k_{0}+1}, \ldots, \underbrace{r_{m}, r_{m} \ldots, r_{m}}_{k_{m}+1})
$$

The potential part in $F$ has contribution from the internal and external momenta. The internal part is denoted by

$$
P(\mathbf{r} ; \underline{\mathbf{q}} ; \mathbf{k}):=\prod_{j=0}^{|\mathbf{k}|} R^{2}\left(r_{j}-q_{j 1}\right) R^{2}\left(r_{j}-q_{j 2}\right) \ldots R^{2}\left(r_{j}-q_{j k_{j}}\right) ;
$$

the external part by

$$
M(\mathbf{r}, m)=\prod_{j=0}^{m-1} R\left(r_{j}-r_{j+1}\right)
$$

Therefore, we have

$$
F(\mathbf{p}, n)=P(\mathbf{r} ; \underline{\mathbf{q}} ; \mathbf{k}) M(\mathbf{r},|\mathbf{k}|) \widehat{\psi}_{0}\left(r_{m}\right)
$$

after renaming the momenta. We can now rewrite $C_{\pi}(2.17)$ for a simple pairing $\pi \in \Pi_{n, n^{\prime}}$ as follows:

$$
\begin{aligned}
C_{\pi}= & \lambda^{2 \bar{n}} \int d \mathbf{r}_{m} d \underline{\mathbf{q}}_{\mathbf{k}} d \underline{\mathbf{q}}_{\mathbf{k}^{\prime}}^{\prime} \overline{Q(t ; \mathbf{r}, \underline{\mathbf{q}} ; \mathbf{k})} Q\left(t ; \mathbf{r}, \underline{\mathbf{q}^{\prime}} ; \mathbf{k}^{\prime}\right) \\
& \times \overline{P(\mathbf{r} ; \underline{\mathbf{q}} ; \mathbf{k}) M(\mathbf{r},|\mathbf{k}|)} P\left(\mathbf{r} ; \underline{\mathbf{q}^{\prime}} ; \mathbf{k}^{\prime}\right) M\left(\mathbf{r},\left|\mathbf{k}^{\prime}\right|\right)\left|\widehat{\psi}_{0}\left(r_{m}\right)\right|^{2},
\end{aligned}
$$


where

$$
d \underline{\mathbf{q}}_{\mathbf{k}}=\prod_{1 \leq \ell \leq k_{j}, 0 \leq j \leq m} d q_{j \ell} \quad d \mathbf{r}_{m}=\prod_{0 \leq j \leq m} d r_{j},
$$

and similarly for $d \underline{\mathbf{q}}_{\mathbf{k}^{\prime}}^{\prime}$.

Recall the K-identity in (3.7). This implies the identity for $Q$ :

$$
Q(t ; \mathbf{r}, \underline{\mathbf{q}} ; \mathbf{k})=i e^{t \eta / 2} \int d \alpha e^{-i \alpha t} \prod_{j=0}^{m}\left[\left\{\frac{1}{\alpha-r_{j}^{2} / 2+i \eta}\right\}^{k_{j}+1} \prod_{\ell=1}^{k_{j}} \frac{1}{\alpha-q_{j \ell}^{2} / 2+i \eta}\right]
$$

We now perform the $q$ and $q^{\prime}$ integration in $C_{\pi}$ in the expression (3.57). Define $\Theta_{\alpha, \eta}(r)$ and $G_{\eta}\left(\alpha, \beta, \mathbf{r}, \mathbf{r}^{\prime}, \mathbf{k}, \mathbf{k}^{\prime}\right)$ by

$$
\begin{gathered}
\Theta_{\alpha, \eta}(r):=\int \frac{R^{2}(r-q)}{\alpha-q^{2} / 2+i \eta} d q \\
G_{\eta}\left(\alpha, \beta, \mathbf{r}, \mathbf{r}^{\prime}, \mathbf{k}, \mathbf{k}^{\prime}\right):=\bar{M}(\mathbf{r}, m) M\left(\mathbf{r}^{\prime}, m\right) \prod_{j=0}^{m}\left[\bar{\Theta}_{\alpha, \eta}\left(r_{j}\right)\right]^{k_{j}}\left[\Theta_{\beta, \eta}\left(r_{j}^{\prime}\right)\right]^{k_{j}^{\prime}} .
\end{gathered}
$$

Notice that $\Theta$ depends on $r$ only through its norm as $R$ is spherically symmetric. We also need the notations later on:

$$
\begin{aligned}
\Theta_{\alpha, 0^{+}}(r) & :=\lim _{\eta \rightarrow 0^{+}} \Theta_{\alpha, \eta}(r), \\
G_{0}\left(\alpha, \beta, \mathbf{r}, \mathbf{r}^{\prime}, \mathbf{k}, \mathbf{k}^{\prime}\right) & :=\lim _{\eta \rightarrow 0^{+}} G_{\eta}\left(\alpha, \beta, \mathbf{r}, \mathbf{r}^{\prime}, \mathbf{k}, \mathbf{k}^{\prime}\right) .
\end{aligned}
$$

Then we have the identity

$$
\begin{aligned}
C_{\pi}= & \lambda^{2 \bar{n}} e^{t \eta} \int d \mathbf{r}_{m} \int_{-\infty}^{\infty} d \alpha d \beta e^{i(\alpha-\beta) t} \\
& \times \prod_{j=0}^{m}\left[\frac{1}{\alpha-r_{j}^{2} / 2-i \eta}\right]^{k_{j}+1}\left[\frac{1}{\beta-r_{j}^{2} / 2+i \eta}\right]^{k_{j}^{\prime}+1} G_{\eta}\left(\alpha, \beta, \mathbf{r}, \mathbf{r}, \mathbf{k}, \mathbf{k}^{\prime}\right)\left|\widehat{\psi}_{0}\left(r_{m}\right)\right|^{2}
\end{aligned}
$$

We shall need several properties of $\Theta_{\alpha, \eta}(r)$ (including the existence of the limit in (3.61)). These are summarized in the following general lemma about singular integral operators. The proof is found in the appendix.

Lemma 3.10 . Let

$$
\left(Y_{z} f\right)(r):=\int \frac{f(r-q)}{z-q^{2}} d q
$$

be a family of linear operators parametrized by a complex parameter $z$ with $0<\operatorname{Im} z<1 / 2$. Let $\alpha:=\operatorname{Re} z$ and $\eta:=\operatorname{Im} z$. 
(i) For $d \geq 3$ we have

$$
\left\|Y_{z} f\right\|_{\infty} \leq(\text { const. })\|f\|_{2 d, 2 d},
$$

and for $d=2$

$$
\left\|Y_{z} f\right\|_{\infty} \leq(\text { const. })\left(1+[\log \alpha]_{-}\right)\|f\|_{2 d, 2 d},
$$

where $[x]_{-}=-\min \{0, x\}$, i.e. the coefficient is $1+|\log \alpha|$ for $\alpha<1$ and is 1 for $\alpha \geq 1$. Here the constants may depend on d but is independent of $\eta$.

(ii) If $z^{\prime}=\alpha^{\prime}+i \eta^{\prime}$ and $\eta \geq \eta^{\prime}$, then for $d \geq 3$

$$
\left\|Y_{z} f-Y_{z^{\prime}} f\right\|_{\infty} \leq(\text { const. })\left|z-z^{\prime}\right| \eta^{-1 / 2}\|f\|_{2 d, 2 d},
$$

and for $d=2$

$$
\left.\left\|Y_{z} f-Y_{z^{\prime}} f\right\|_{\infty} \leq \text { (const. }\right)\left|z-z^{\prime}\right|\left(\eta^{-1 / 2}+\frac{1}{|z|}+\frac{1}{\left|z^{\prime}\right|}\right)\|f\|_{2 d, 2 d} .
$$

Remark: Obviously

$$
\Theta_{\alpha, \eta}(r)=2\left[Y_{2(\alpha+i \eta)}\left(R^{2}\right)\right](r),
$$

hence the existence of the limit (3.61) follows from (3.66). Since

$$
\left\|R^{2}(r-\cdot)-R^{2}\left(r^{\prime}-\cdot\right)\right\|_{2 d, 2 d} \leq C\left|r-r^{\prime}\right|
$$

with $C$ depending on the Schwarz norm of $R$, we also have from (3.64) that for $d \geq 3$

$$
\left|\Theta_{\alpha, \eta}(r)-\Theta_{\alpha, \eta}\left(r^{\prime}\right)\right| \leq C\left|r-r^{\prime}\right| .
$$

In $d=2$ the estimate (3.69) is valid with an extra factor $\left(1+[\log \alpha]_{-}\right)$.

Proof of Lemma 3.7.

We first derive the following apriori estimate for $C_{\pi}$. This estimate is better than Lemma 3.4 by removing the factors $\log t$, but it still does not recover the $1 / n$ !.

Lemma 3.11 . If $\pi \in \Pi_{n, n^{\prime}}$ is a simple pairing, then

$$
\left|C_{\pi}\right| \leq\left(C \lambda^{2} t\right)^{\bar{n}} .
$$

Proof of Lemma 3.11. We present the proof for $d \geq 3$. The case $d=2$ requires some technical modifications which are explained in Section 6. It is clear from (3.64) that

$$
\left|\Theta_{\alpha, \eta}(r)\right| \leq C
$$


uniformly in $\alpha, \eta$ and $r$. Hence, by (1.9),

$$
\left|G_{\eta}\left(\alpha, \beta, \mathbf{r}, \mathbf{r}, \mathbf{k}, \mathbf{k}^{\prime}\right)\right|\left|\widehat{\psi}_{0}\left(r_{m}\right)\right|^{2} d \mathbf{r}_{m} \leq C^{\|\mathbf{k}\|+\left\|\mathbf{k}^{\prime}\right\|+2 m} d \mu\left(\mathbf{r}_{m}\right)
$$

with a constant independent of $\eta, \alpha, \beta$. Recall the definition of $\Sigma(t, \mathbf{r}, \mathbf{k})$ in (3.26). Thus (3.63) implies that $\left(\eta=t^{-1}\right)$

$$
\left|C_{\pi}\right| \leq(C \lambda)^{2 \bar{n}} e^{t \eta} \int d \mu\left(\mathbf{r}_{m}\right) \Sigma(t, \mathbf{r}, \mathbf{k}) \Sigma\left(t, \mathbf{r}, \mathbf{k}^{\prime}\right)
$$

By the Schwarz inequality

$$
\Sigma(t, \mathbf{r}, \mathbf{k}) \Sigma\left(t, \mathbf{r}, \mathbf{k}^{\prime}\right) \leq t^{\|\mathbf{k}\|-\left\|\mathbf{k}^{\prime}\right\|} \Sigma^{2}\left(t, \mathbf{r}, \mathbf{k}^{\prime}\right)+t^{\left\|\mathbf{k}^{\prime}\right\|-\|\mathbf{k}\|} \Sigma^{2}(t, \mathbf{r}, \mathbf{k})
$$

We conclude the proof of Lemma 3.11 from the estimate of $\Sigma(t, \mathbf{r}, \mathbf{k})$ in $(3.28)$ with $a=0$.

The estimate in (3.70) for a simple pairing is not optimal and there is an extra $1 / n$ ! missing. We now recover this factor partially. Notice that the $d \alpha d \beta$ integrations in (3.63) could be performed more precisely if $G$ were independent of $\alpha, \beta$. This dependence however is very mild and can be removed. Thus the main term will have no $\alpha, \beta$ dependence and their integrations can be done explicitly.

Recall the definition $\Theta_{\alpha, 0^{+}}$in (3.61). Define

$$
\Theta(v):=\Theta_{v^{2} / 2,0^{+}}(v)
$$

Lemma 3.12 . We have the following representation for $C_{\pi}, \pi$ simple;

$$
\begin{gathered}
C_{\pi}=\lambda^{2 \bar{n}} \int d \mathbf{r}_{m} \overline{Q^{*}(t ; \mathbf{r} ; \mathbf{k})} Q^{*}\left(t ; \mathbf{r} ; \mathbf{k}^{\prime}\right)|M(\mathbf{r}, m)|^{2} \overline{\Theta\left(v_{0}\right)}{ }^{\|\mathbf{k}\|} \Theta\left(v_{0}\right)^{\left\|\mathbf{k}^{\prime}\right\|}\left|\widehat{\psi}_{0}\left(r_{m}\right)\right|^{2} \\
+O\left(\left(C_{a} \lambda^{2} t\right)^{\bar{n}} t^{-\frac{a}{2}}\right)
\end{gathered}
$$

for any $0 \leq a<1$.

Proof of Lemma 3.12. Again, the proof is presented for $d \geq 3$. The case $d=2$ is referred to Section 6. We claim that $G_{\eta}$ in the formula (3.63) can be replaced by $|M(\mathbf{r}, m)|^{2} \overline{\Theta\left(r_{0}\right)}\|\mathbf{k}\| \mid \Theta\left(r_{0}\right)^{\left\|\mathbf{k}^{\prime}\right\|}$ with small errors. Assuming this replacement in (3.63), we can use the $Q$-identity (3.58) with no $\mathbf{q}$ to integrate the $\alpha$ and $\beta$ in (3.63). This proves the main term in (3.75). 
From the definition of $C_{\pi}$ in (3.63), the error from the replacement is bounded by

$$
\begin{aligned}
& C \lambda^{2 \bar{n}} \int d \mathbf{r}_{m} \int_{-\infty}^{\infty} d \alpha d \beta \prod_{j=0}^{m}\left|\frac{1}{\alpha-r_{j}^{2} / 2-i \eta}\right|^{k_{j}+1}\left|\frac{1}{\beta-r_{j}^{2} / 2+i \eta}\right|^{k_{j}^{\prime}+1}
\end{aligned}
$$

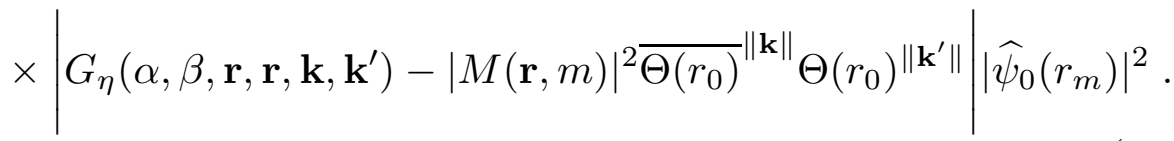

Recall the definition of $G$ in (3.60). Then the difference in (3.76) is equal to

$$
\begin{gathered}
G_{\eta}\left(\alpha, \beta, \mathbf{r}, \mathbf{r}, \mathbf{k}, \mathbf{k}^{\prime}\right)-|M(\mathbf{r}, m)|^{2}{\overline{\Theta\left(v_{0}\right)}}^{\|\mathbf{k}\|} \Theta\left(v_{0}\right)^{\left\|\mathbf{k}^{\prime}\right\|} \\
=|M(\mathbf{r}, m)|^{2}\left\{\prod_{j=0}^{m}\left[\bar{\Theta}_{\alpha, \eta}\left(r_{j}\right)\right]^{k_{j}}\left[\Theta_{\beta, \eta}\left(r_{j}\right)\right]^{k_{j}^{\prime}}-{\overline{\Theta\left(r_{0}\right)}}^{\|\mathbf{k}\|} \Theta\left(r_{0}\right)^{\left\|\mathbf{k}^{\prime}\right\|}\right\} .
\end{gathered}
$$

Recall that $\Theta_{\alpha, \eta}(r)$ depends only on $|r|$, hence the function $\Theta_{\alpha, \eta}^{*}(|r|):=\Theta_{\alpha, \eta}(r)$ is well defined. The inequality (3.69) is valid for $\Theta^{*}$ as well:

$$
\left|\Theta_{\alpha, \eta}^{*}(|r|)-\Theta_{\alpha, \eta}^{*}\left(\left|r^{\prime}\right|\right)\right| \leq C|| r|-| r^{\prime}||
$$

Now we have the following estimate

$$
\begin{aligned}
\Theta_{\alpha, \eta}\left(r_{j}\right)-\Theta\left(r_{0}\right)= & {\left[\Theta_{\alpha, \eta}^{*}\left(\left|r_{j}\right|\right)-\Theta_{\alpha, \eta}^{*}(\sqrt{2|\alpha|})\right]+\left[\Theta_{\alpha, \eta}^{*}(\sqrt{2|\alpha|})-\Theta_{\alpha, \eta}^{*}\left(\left|r_{0}\right|\right)\right] } \\
& +\left[\Theta_{\alpha, \eta}^{*}\left(\left|r_{0}\right|\right)-\Theta_{r_{0}^{2} / 2, \eta}^{*}\left(\left|r_{0}\right|\right)\right]+\left[\Theta_{r_{0}^{2} / 2, \eta}^{*}\left(\left|r_{0}\right|\right)-\Theta_{r_{0}^{2} / 2,0^{+}}^{*}\left(\left|r_{0}\right|\right)\right] \\
\leq & C\left(|| r_{j}|-\sqrt{2|\alpha|}|+|| r_{0}|-\sqrt{2|\alpha|}|\right)+C \eta^{-1 / 2}\left(\left|\alpha-r_{0}^{2} / 2\right|+\eta\right) \\
\leq & C\left(|\alpha|^{-1 / 2}+\eta^{-1 / 2}\right)\left(\left|\alpha-\frac{r_{j}^{2}}{2}\right|+\left|\alpha-\frac{r_{0}^{2}}{2}\right|\right)+C \eta^{1 / 2} .
\end{aligned}
$$

Similar estimate is valid for $\Theta_{\beta, \eta}\left(r_{j}\right)$. Recall that $\eta=1 / t$. Using the uniform bound on $\Theta$ and a telescopic sum to bound the curely bracket in (3.77) we can bound (3.76) by

$$
\begin{aligned}
& C^{\bar{n}} \int d \mu\left(\mathbf{r}_{m}\right) \int_{-\infty}^{\infty} d \alpha d \beta \prod_{j=0}^{m}\left|\frac{1}{\alpha-r_{j}^{2} / 2-i \eta}\right|^{k_{j}+1}\left|\frac{1}{\beta-r_{j}^{2} / 2+i \eta}\right|^{k_{j}^{\prime}+1}\left[\eta^{-1 / 2}+|\alpha|^{-1 / 2}+|\beta|^{-1 / 2}\right] \\
& \times\left[O\left(\alpha-r_{0}^{2} / 2\right)+O\left(\beta-r_{0}^{2} / 2\right)+\sum_{j}\left\{O\left(\alpha-r_{j}^{2} / 2\right)+O\left(\beta-r_{j}^{2} / 2\right)+O(\eta)\right\}\right] .
\end{aligned}
$$

Suppose first that the term $\left[\eta^{-1 / 2}+|\alpha|^{-1 / 2}+|\beta|^{-1 / 2}\right]$ were not present. The error from $O\left(\alpha-r_{j}^{2} / 2\right)$ can be bounded in a similar way as $O\left(\alpha-r_{0}^{2} / 2\right)$. So we shall focus only on 
$O\left(\alpha-r_{0}^{2} / 2\right)$. The net effect of the term $O\left(\alpha-r_{0}^{2} / 2\right)$ is that it decreases $k_{0}$ by 1 . By the proof of Lemma 3.11, this gives an extra $t^{-1}$ factor unless $k_{0}=0$. In this case

$$
\left|\frac{1}{\alpha-r_{0}^{2} / 2-i \eta}\right| \times O\left(\alpha-r_{0}^{2} / 2\right)
$$

is bounded, and thus the $d r_{0}$ integration gives a factor $t^{k_{0}^{\prime}}$. We use the argument from the proof of Lemma 3.11 to bound the integration of the other momenta $r_{1}, \cdots, r_{m}$. Again, we obtain an extra $t^{-1}$ factor. The estimate for the $O\left(\beta-r_{0}^{2} / 2\right)$ term is identical. Finally the $O(\eta)$ term gives immediately a factor $t^{-1}$ from its choice. Hence the net effect of the terms in the second square bracket is a factor $t^{-1}$ compared with the main term.

Now we include the factor $\left[\eta^{-1 / 2}+|\alpha|^{-1 / 2}+|\beta|^{-1 / 2}\right]$ as well. We claim that the net effect of this factor is estimated by $C_{a}^{\bar{n}} \eta^{-1+\frac{a}{2}}$ for any $0 \leq a<1$, hence (3.80) has an extra factor $t^{-1} \eta^{-1+\frac{a}{2}}=t^{-\frac{a}{2}}$ compared with the main term. This claim is clear for the $\eta^{-1 / 2}$ term. Now we consider the term $|\alpha|^{-1 / 2}$, the term with $|\beta|^{-1 / 2}$ is identical. We follow the argument in the previous paragraph. When referring to Lemma 3.11, we need to consider the quantity

$$
\widetilde{\Sigma}(t, \mathbf{p}, \mathbf{k}):=\int_{-\infty}^{\infty} \frac{d \alpha}{\sqrt{|\alpha|}} \prod_{j=0}^{m}\left|\frac{1}{\alpha-p_{j}^{2} / 2-i t^{-1}}\right|^{k_{j}+1} .
$$

instead of $\Sigma(t, \mathbf{p}, \mathbf{k})$. We need the following modification of the estimate (3.28)

$$
\int|\widetilde{\Sigma}(t, \mathbf{p}, \mathbf{k})|^{2-a} d \mu\left(\mathbf{p}_{m}\right) \leq t^{1-\frac{a}{2}}\left(C_{a} t\right)^{(1-a) m+(2-a)\|\mathbf{k}\|}
$$

for any $0 \leq a<1$. Combined with the trivial bound $|\widetilde{\Sigma}(t, \mathbf{p}, \mathbf{k})| \leq(C t)^{m+\|\mathbf{k}\|}$ we obtain

$$
\int|\widetilde{\Sigma}(t, \mathbf{p}, \mathbf{k})|^{2} d \mu\left(\mathbf{p}_{m}\right) \leq t^{1-\frac{a}{2}}\left(C_{a} t\right)^{m+2\|\mathbf{k}\|}
$$

The rest of the proof of Lemma 3.11 is unchanged.

The proof of (3.81) is similar to that of (3.28). The only modification is to change $R\left(\alpha, p_{n-1}, p_{n}\right)$ in $(3.17)$ to

$$
\widetilde{R}\left(\alpha, p_{n-1}, p_{n}\right):=\sqrt{|\alpha|} R\left(\alpha, p_{n-1}, p_{n}\right)
$$

and to change $Z\left(p_{n-1}, p_{n}\right)$ in $(3.18)$ to

$$
\widetilde{Z}\left(p_{n-1}, p_{n}\right):=\int_{-\infty}^{\infty} \frac{d \alpha}{\sqrt{|\alpha|}\left|\alpha-p_{n-1}^{2} / 2+i \eta\right|\left|\beta-p_{n}^{2} / 2+i \eta\right|} .
$$


Simple calculation shows that the estimate (3.19) is valid for $\widetilde{Z}$ with an extra $\eta^{-1 / 2}$ factor on the right hand side. This shows that the term $|\alpha|^{-1 / 2}$ in (3.80) is effectively a factor $\left(\eta^{-1 / 2}\right)^{2-a}$ in the final result as we claimed. This proves Lemma 3.12.

To finish the proof of Lemma 3.7, we now estimate the main term on the right hand side of (3.75). By the Schwarz inequality,

$$
\begin{aligned}
\left|\overline{Q^{*}(t ; \mathbf{r} ; \mathbf{k})} Q^{*}\left(t ; \mathbf{r} ; \mathbf{k}^{\prime}\right)\right| \leq & t^{\left\|\mathbf{k}^{\prime}\right\|-\|\mathbf{k}\|}\left[\frac{(m+\|\mathbf{k}\|) !}{\left(m+\left\|\mathbf{k}^{\prime}\right\|\right) !}\right]^{1 / 2} \mid \overline{\left.Q^{*}(t ; \mathbf{r} ; \mathbf{k})\right|^{2}} \\
& +t^{\|\mathbf{k}\|-\left\|\mathbf{k}^{\prime}\right\|}\left[\frac{\left(m+\left\|\mathbf{k}^{\prime}\right\|\right) !}{(m+\|\mathbf{k}\|) !}\right]^{1 / 2}\left|Q^{*}\left(t ; \mathbf{r} ; \mathbf{k}^{\prime}\right)\right|^{2},
\end{aligned}
$$

We now use the estimate (3.27) with $a=1 / 2$ and (3.72) to have

$$
\left|C_{\pi}\right| \leq \frac{\left(C \lambda^{2} t\right)^{\bar{n}}}{\left[(m+\|\mathbf{k}\|) !\left(m+\left\|\mathbf{k}^{\prime}\right\|\right) !\right]^{1 / 2}}+O\left(\left(C_{a} \lambda^{2} t\right)^{\bar{n}} t^{-\frac{a}{2}}\right) .
$$

Finally, using that $m+\|\mathbf{k}\| \geq n / 2, m+\left\|\mathbf{k}^{\prime}\right\| \geq n^{\prime} / 2$ and $n ! \leq C^{\bar{n}}\left[\left(n^{\prime} / 2\right) !\right][(n / 2) !]$, we obtain the statement of Lemma 3.7.

\section{Wigner transform of the main term.}

As it is explained in Step 2.1, we have to compute the Wigner transform of the main term

$$
\psi_{t, M}^{\text {main }}:=\sum_{n=0}^{M-1} \psi_{n}(t)
$$

Recall $\widehat{J}_{\varepsilon}(x, v)=\varepsilon^{-d} \widehat{J}\left(\xi \varepsilon^{-1}, v\right)$. The goal of this Section is to compute

$$
\mathbf{E} \int \varepsilon^{-d} \overline{\widehat{J}\left(\xi \varepsilon^{-1}, v\right)} \widehat{W}_{\psi_{T / \varepsilon, M}^{\text {main }}}(\xi, v) d \xi d v=\mathbf{E} \int{\widehat{\widehat{J}_{\varepsilon}(\xi, v)}}_{\widehat{\psi}_{T / \varepsilon, M}^{\text {main }}}\left(v-\frac{\xi}{2}\right) \widehat{\psi}_{T / \varepsilon, M}^{\text {main }}\left(v+\frac{\xi}{2}\right) d \xi d v
$$

in the limit $\lim _{M \rightarrow \infty} \lim _{\varepsilon \rightarrow 0}$. By definition of $U^{\Gamma}$ in $(2.12)$ and $C_{\pi}^{\Gamma}$ in (2.16), we have

$$
\mathbf{E} \int \varepsilon^{-d} \overline{\widehat{J}\left(\xi \varepsilon^{-1}, v\right)} \widehat{W}_{\psi_{T / \varepsilon, M}^{\operatorname{main}}}(\xi, v) d \xi d v=\sum_{n, n^{\prime}=0}^{M-1} U_{n, n^{\prime}}^{\widehat{J}_{\varepsilon}}=\sum_{n, n^{\prime}=0}^{M-1} \sum_{\pi \in \Pi_{n, n^{\prime}}} C_{\pi}^{\widehat{J}_{\varepsilon}} .
$$

If we put $\widehat{J}_{\varepsilon}(\xi, v)$ to be a delta function in $\xi, C_{\pi}^{\widehat{J}_{\varepsilon}}$ becomes $C_{\pi}$ computed in Section 3. The conclusions were that $C_{\pi}$ is of lower order if $\pi$ is crossing or nested and it can be written 
as (3.75) if $\pi$ is simple. Furthermore, for simple pairing it is bounded by (3.49) and (3.70). All these estimates can be extended to $C_{\pi}^{\widehat{J}_{\varepsilon}}$ as well, uniformly in $\varepsilon$. We now state them and comment on the modifications needed for the proofs. Since $J$ is fixed, we can introduce the simplifying notation

$$
C_{\pi}^{\varepsilon}:=C_{\pi}^{\widehat{J}_{\varepsilon}}, \quad U_{n, n^{\prime}}^{\varepsilon}:=U_{n, n^{\prime}}^{\widehat{J}_{\varepsilon}} .
$$

Lemma 4.1 . The contributions from the crossing and nested pairings are negligible in the sense that

$$
U_{n, n^{\prime}}^{\varepsilon}=\sum_{\substack{\pi \in \Pi_{n, n^{\prime}} \\ \pi \text { simple }}} C_{\pi}^{\varepsilon}+O\left(\bar{n} !\left(C \lambda^{2} t\right)^{\bar{n}} t^{-1}(\log t)^{\bar{n}+3}\right) .
$$

In particular for any $M$ fixed we have

$$
\lim _{\varepsilon \rightarrow 0} \sum_{n, n^{\prime}=0}^{M-1} U_{n, n^{\prime}}^{\varepsilon}=\lim _{\varepsilon \rightarrow 0} \sum_{n, n^{\prime}=0}^{M-1} \sum_{\substack{\pi \in \Pi_{n, n^{\prime}} \\ \pi \text { simple }}} C_{\pi}^{\varepsilon} .
$$

Since $t=T \varepsilon^{-1}, \lambda=\sqrt{\varepsilon}$.

Proof of Lemma 4.1. The proofs of the apriori estimate Lemma 3.4 and Lemma 3.5 can be carried out with only minor changes. We only need to replace the term

$$
\frac{1}{\left|\alpha-w_{0}^{2} / 2+i t^{-1}\right|\left|\beta-w_{0}^{2} / 2+i t^{-1}\right|}
$$

in (3.31) and (3.37) by

$$
\frac{1}{\left|\alpha-\frac{1}{2}\left(\widetilde{w}_{0}-\frac{\xi}{2}\right)^{2}+i t^{-1}\right|\left|\beta-\frac{1}{2}\left(\widetilde{w}_{0}+\frac{\xi}{2}\right)^{2}+i t^{-1}\right|}
$$

with $\widetilde{w}_{0}:=w_{0}+\xi / 2$. The rest is the same. The proof for the nested pairings is essentially unchanged (cf. Lemma 3.6).

We can generalize (3.63) for a simple pairing $\pi \in \Pi_{n, n^{\prime}}$ to include the test function $J$ :

$$
\begin{aligned}
C_{\pi}^{\varepsilon}= & \lambda^{n+n^{\prime}} e^{t \eta} \int d \xi \int d \mathbf{v}_{m} \overline{\widehat{J}_{\varepsilon}\left(\xi, v_{0}\right)} \int_{-\infty}^{\infty} d \alpha d \beta e^{i(\alpha-\beta) t} \\
& \times \prod_{j=0}^{m}\left[\frac{1}{\alpha-\frac{1}{2}\left(v_{j}-\frac{\xi}{2}\right)^{2}-i \eta}\right]^{k_{j}+1}\left[\frac{1}{\beta-\frac{1}{2}\left(v_{j}+\frac{\xi}{2}\right)^{2}+i \eta}\right]^{k_{j}^{\prime}+1} \\
& \times G_{\eta}\left(\alpha, \beta, \mathbf{v}-\frac{\xi}{2}, \mathbf{v}+\frac{\xi}{2}, \mathbf{k}, \mathbf{k}^{\prime}\right) \widehat{\widehat{\psi}}_{0}\left(v_{m}-\frac{\xi}{2}\right) \widehat{\psi}_{0}\left(v_{m}+\frac{\xi}{2}\right)
\end{aligned}
$$


where

$$
(\mathbf{v} \pm \xi / 2)_{j}=v_{j} \pm \xi / 2 .
$$

Recall also that

$$
n=2\|\mathbf{k}\|+m, n^{\prime}=2\left\|\mathbf{k}^{\prime}\right\|+m, \quad|\mathbf{k}|=\left|\mathbf{k}^{\prime}\right|=m .
$$

The apriori estimate for $C_{\pi}$, Lemma 3.11 , can be extended to $C_{\pi}^{\varepsilon}$ :

Lemma 4.2 . For any simple pairing $\pi \in \Pi_{n, n^{\prime}}$

$$
\left|C_{\pi}^{\varepsilon}\right| \leq\left(C \lambda^{2} t\right)^{\bar{n}}
$$

Proof of Lemma 4.2. The proof is almost identical to that of Lemma 3.11. We can bound $\left|C_{\pi}^{\varepsilon}\right|$ by

$$
\begin{aligned}
\left|C_{\pi}^{\varepsilon}\right| \leq & \left(C \lambda^{2}\right)^{\bar{n}} \int d \xi d \mathbf{v}_{m}\left|\widehat{J}_{\varepsilon}\left(\xi, v_{0}\right)\right| \Sigma\left(t ; \mathbf{v}-\frac{\xi}{2}, \mathbf{k}\right) \Sigma\left(t ; \mathbf{v}+\frac{\xi}{2}, \mathbf{k}^{\prime}\right) \\
& \times \sup _{\alpha, \beta}\left|G_{\eta}\left(\alpha, \beta, \mathbf{v}-\frac{\xi}{2}, \mathbf{v}+\frac{\xi}{2}, \mathbf{k}, \mathbf{k}^{\prime}\right)\right||| \widehat{\widehat{\psi}}_{0}\left(v_{m}-\frac{\xi}{2}\right) \widehat{\psi}_{0}\left(v_{m}+\frac{\xi}{2}\right) \mid .
\end{aligned}
$$

Recalling the definition of $\Phi(2.14)$, we have by a Schwarz inequality similar to (3.73)

$$
\begin{gathered}
\Sigma\left(t ; \mathbf{v}-\frac{\xi}{2}, \mathbf{k}\right) \Sigma\left(t ; \mathbf{v}+\frac{\xi}{2}, \mathbf{k}^{\prime}\right)\left|\overline{\widehat{\psi}_{0}\left(v_{m}-\frac{\xi}{2}\right)} \widehat{\psi}_{0}\left(v_{m}+\frac{\xi}{2}\right)\right| \\
\leq t^{\left\|\mathbf{k}^{\prime}\right\|-\|\mathbf{k}\|} \frac{\Sigma^{2}\left(t ; \mathbf{v}-\frac{\xi}{2}, \mathbf{k}\right) \Phi^{2}\left(v_{m}-\frac{\xi}{2}\right)}{<v_{m}-\xi / 2>^{20 d}}+t^{\|\mathbf{k}\|-\left\|\mathbf{k}^{\prime}\right\|} \frac{\Sigma^{2}\left(t ; \mathbf{v}+\frac{\xi}{2}, \mathbf{k}^{\prime}\right) \Phi^{2}\left(v_{m}+\frac{\xi}{2}\right)}{<v_{m}+\xi / 2>^{20 d}} .
\end{gathered}
$$

By definition of $G_{\eta}$ in (3.60), we have the simple bound

$$
\sup _{\alpha, \beta}\left|G_{\eta}\left(\alpha, \beta, \mathbf{v}-\frac{\xi}{2}, \mathbf{v}+\frac{\xi}{2}, \mathbf{k}, \mathbf{k}^{\prime}\right)\right| \leq \prod_{j=0}^{m-1} \frac{C}{\left\langle\left(v_{j} \pm \frac{\xi}{2}\right)-\left(v_{j+1} \pm \frac{\xi}{2}\right)\right\rangle^{20 d}} .
$$

Recall the definition of the measure $\mu$ in (3.4). From the last two bounds, we have the following bound similar to (3.72) in the proof of Lemma 3.11:

$$
\begin{aligned}
& \sup _{\alpha, \beta} \Sigma\left(t ; \mathbf{v}-\frac{\xi}{2}, \mathbf{k}\right) \Sigma\left(t ; \mathbf{v}+\frac{\xi}{2}, \mathbf{k}^{\prime}\right)\left|G_{\eta}\left(\alpha, \beta, \mathbf{v}-\frac{\xi}{2}, \mathbf{v}+\frac{\xi}{2}, \mathbf{k}, \mathbf{k}^{\prime}\right)\right|\left|\overline{\widehat{\psi}_{0}\left(v_{m}-\frac{\xi}{2}\right)} \widehat{\psi}_{0}\left(v_{m}+\frac{\xi}{2}\right)\right| d \mathbf{v}_{m} \\
& \leq C^{m}\left[t^{\left\|\mathbf{k}^{\prime}\right\|-\|\mathbf{k}\|} \Sigma^{2}\left(t ; \mathbf{v}-\frac{\xi}{2}, \mathbf{k}\right) d \mu\left(\mathbf{v}_{m}-\frac{\xi}{2}\right)+t^{\|\mathbf{k}\|-\left\|\mathbf{k}^{\prime}\right\|} \Sigma^{2}\left(t ; \mathbf{v}+\frac{\xi}{2}, \mathbf{k}^{\prime}\right) d \mu\left(\mathbf{v}_{m}+\frac{\xi}{2}\right)\right] .
\end{aligned}
$$


Hence we can bound $\left|C_{\pi}^{\varepsilon}\right|$ by

$$
\begin{aligned}
\left|C_{\pi}^{\varepsilon}\right| \leq & \left(C \lambda^{2}\right)^{\bar{n}} t^{\left\|\mathbf{k}^{\prime}\right\|-\|\mathbf{k}\|} \int d \xi d \mu\left(\mathbf{v}_{m}-\frac{\xi}{2}\right)\left|\widehat{J}_{\varepsilon}\left(\xi, v_{0}\right)\right| \Sigma^{2}\left(t ; \mathbf{v}-\frac{\xi}{2}, \mathbf{k}\right) \\
& +\left(C \lambda^{2}\right)^{\bar{n}} t^{\|\mathbf{k}\|-\left\|\mathbf{k}^{\prime}\right\|} \int d \xi d \mu\left(\mathbf{v}_{m}+\frac{\xi}{2}\right)\left|\widehat{J}_{\varepsilon}\left(\xi, v_{0}\right)\right| \Sigma^{2}\left(t ; \mathbf{v}+\frac{\xi}{2}, \mathbf{k}^{\prime}\right) .
\end{aligned}
$$

Using that $\int d \xi \sup _{v}\left|\widehat{J}_{\varepsilon}(\xi, v)\right|$ is bounded uniformly in $\varepsilon$, we only have to perform the $\mathbf{v}$ integration. After a change of variables $\mathbf{v} \rightarrow \mathbf{v} \pm \xi / 2$ we can use (3.28) with $a=0$. This concludes the proof of (4.4).

We have the following Lemma parallel to the Lemma 3.12:

Lemma 4.3 . We have the following representation for $C_{\pi}^{\varepsilon}$ for a simple pairing $\pi \in \Pi_{n, n^{\prime}}$ :

$$
C_{\pi}^{\varepsilon}=C_{\pi}^{\varepsilon, \text { main }}+O\left(\left(C_{a} \lambda^{2} t\right)^{\bar{n}} t^{-\frac{a}{2}}\right)
$$

$(0 \leq a<1)$ with

$$
C_{\pi}^{\varepsilon, \text { main }}:=\int d \xi \int d \mathbf{v}_{m} \overline{\widehat{J}_{\varepsilon}\left(\xi, v_{0}\right)} Y\left(\xi, \mathbf{v}, \mathbf{k}, \mathbf{k}^{\prime}\right)
$$

where

$$
\begin{aligned}
Y\left(\xi, \mathbf{v}, \mathbf{k}, \mathbf{k}^{\prime}\right)= & \lambda^{2 m+2\|\mathbf{k}\|+2\left\|\mathbf{k}^{\prime}\right\| \overline{\Theta\left(v_{0}\right)}}{ }^{\|\mathbf{k}\|} \Theta\left(v_{0}\right)^{\left\|\mathbf{k}^{\prime}\right\|}|M(\mathbf{v}, m)|^{2} \\
& \times \overline{Q^{*}\left(t ; \mathbf{v}-\frac{\xi}{2} ; \mathbf{k}\right)} Q^{*}\left(t ; \mathbf{v}+\frac{\xi}{2} ; \mathbf{k}^{\prime}\right) \widehat{W}_{\psi_{0}}\left(\xi, v_{m}\right) .
\end{aligned}
$$

Proof of Lemma 4.3. The proof is parallel to that of Lemma 3.12. We shall only note the difference in $d \geq 3$. The term similar to (3.77) is now bounded by

$$
\begin{gathered}
G_{\eta}\left(\alpha, \beta, \mathbf{v}-\frac{\xi}{2}, \mathbf{v}+\frac{\xi}{2}, \mathbf{k}, \mathbf{k}^{\prime}\right)-|M(\mathbf{v}, m)|^{2} \prod_{j=0}^{m} \overline{\Theta\left(v_{0}\right)}{ }^{k_{j}} \Theta\left(v_{0}\right)^{k_{j}^{\prime}} \\
\leq C^{m}\left[\eta^{-1 / 2}+|\alpha|^{-1 / 2}+|\beta|^{-1 / 2}\right]\left[O(\xi)+O(\eta)+\sum_{j=0}^{m} O\left(\alpha-\frac{v_{j}^{2}}{2}\right)+\sum_{j=0}^{m} O\left(\beta-\frac{v_{j}^{2}}{2}\right)\right]|M(\mathbf{v}, m)|^{2} .
\end{gathered}
$$

Recall again the simple bound (2.9) for the pairing between Wigner transform and a test function. We can now follow the proof of Lemma 3.12 to bound the last three terms in the second square bracket in (4.9). Since $\widehat{J}$ decays sufficiently fast in $\xi$, we have

$$
\int d \xi \sup _{v}\left|\widehat{J}_{\varepsilon}(\xi, v)\right||\xi|=\int d \xi \varepsilon^{-d} \sup _{v}\left|\widehat{J}\left(\xi \varepsilon^{-1}, v\right)\right||\xi| \leq \varepsilon=C t^{-1}
$$

This provides the bound needed for the new error term $O(\xi)$.

We have all the ingredients for the following Lemma which is parallel to Lemma 3.7. The proof is the same and we omit it. 
Lemma 4.4 . We have the following estimate on $C_{\pi}^{\varepsilon, \text { main }}$ for a simple $\pi \in \Pi_{n, n^{\prime}}$ uniformly in $\varepsilon$ :

$$
\left|C_{\pi}^{\varepsilon, \text { main }}\right| \leq \frac{\left(C \lambda^{2} t\right)^{\bar{n}}}{(\bar{n} !)^{1 / 2}}
$$

Combining Lemma 4.1 and Lemma 4.3, we have thus proved that for any $M$ fixed

$$
\lim _{\varepsilon \rightarrow 0} \sum_{n, n^{\prime}=0}^{M-1} U_{n, n^{\prime}}^{\varepsilon}=\lim _{\varepsilon \rightarrow 0} \sum_{n, n^{\prime}=0}^{M-1} \sum_{\substack{\pi \in \Pi_{n, n} \\ \pi \text { simple }}} C_{\pi}^{\varepsilon, \text { main }} .
$$

We now sum up $C_{\pi}^{\varepsilon, m a i n}$ for all pairings with a fixed number of external momenta $m+1$. Recall that $m$ depends on $\pi$ and we use the notation $m=|\pi|$. Since we have a cutoff in $M$, not all of these pairings (with a fixed number of external momenta $m+1$ ) appeared in (4.11). But the number of simple pairings in $\Pi_{n, n^{\prime}}$ is at most $C^{\bar{n}}$. Hence for any $M$ fixed, we have from Lemma 4.4,

$$
\left|\sum_{m=0}^{M-1} \sum_{\substack{\pi:|\pi|=m \\ \pi \text { simple }}} C_{\pi}^{\varepsilon, \text { main }}-\sum_{n, n^{\prime}=0}^{M-1} \sum_{\substack{\pi \in \Pi_{n, n^{\prime}} \\ \pi \text { simple }}} C_{\pi}^{\varepsilon, \text { main }}\right| \leq \frac{\left(C \lambda^{2} t\right)^{M}}{(M !)^{1 / 8}} .
$$

We can thus extend the summation to infinity with negligible errors (recall that $\lambda^{2} t=T$ which is fixed). The conclusion of Lemma 4.1, 4.3 and 4.4 is

$$
\lim _{M \rightarrow \infty} \lim _{\varepsilon \rightarrow 0} \sum_{n, n^{\prime}=0}^{M-1} U_{n, n^{\prime}}^{\varepsilon}=\lim _{M \rightarrow \infty} \lim _{\varepsilon \rightarrow 0} \sum_{\substack { m=0 \\
\begin{subarray}{c}{\pi \\
\pi \text { simple }{ m = 0 \\
\begin{subarray} { c } { \pi \\
\pi \text { simple } } }\end{subarray}}^{M-1} \sum_{\substack{\text { simplem } \\
\text { s.main }}} C^{\varepsilon, \text { main }}
$$

From the definition of $C_{\pi}^{\varepsilon, m a i n}$ in (4.8), we have for any $m$ fixed

$$
\sum_{\substack{\pi ;|\pi|=m \\ \pi \text { simple }}} C_{\pi}^{\varepsilon, \text { main }}=\sum_{\substack{\mathbf{k}, \mathbf{k}^{\prime} \\|\mathbf{k}|=\left|\mathbf{k}^{\prime}\right|=m}} \int d \xi \int d \mathbf{v}_{m} \overline{\widehat{J}_{\varepsilon}\left(\xi, v_{0}\right)} Y\left(\xi, \mathbf{v}, \mathbf{k}, \mathbf{k}^{\prime}\right) .
$$

We now sum up $\mathbf{k}, \mathbf{k}^{\prime}$. For any $m$ and $a$, there is an identity

$$
\sum_{\mathbf{k}:|\mathbf{k}|=m} a^{\|\mathbf{k}\|} Q^{*}(t ; \mathbf{r} ; \mathbf{k})=e^{-i t a} K(t ; \mathbf{r}, m)
$$

We now give a proof of this identity. By the semigroup property (3.53) and the definition (3.25) of $Q^{*}$, we have

$$
\sum_{\mathbf{k}:|\mathbf{k}|=m} a^{\|\mathbf{k}\|} Q^{*}(t ; \mathbf{r} ; \mathbf{k})=(-i)^{m} \int_{0}^{t}\left(\prod_{j=0}^{m} d s_{j}\right) \delta\left(t-\sum_{j=0}^{m} s_{j}\right) \prod_{j=0}^{m} \sum_{k_{j}=0}^{\infty} a^{k_{j}} K(s_{j} ; \underbrace{r_{j}, r_{j}, \ldots, r_{j}}_{k_{j}+1}) .
$$


We have the simple identity

$$
\sum_{k_{j}=0}^{\infty} a^{k_{j}} K(s_{j} ; \underbrace{r_{j}, r_{j}, \ldots, r_{j}}_{k_{j}+1})=\sum_{k_{j}=0}^{\infty} \frac{\left(-i s_{j} a\right)^{k_{j}}}{k_{j} !} e^{-i s_{j} r_{j}^{2} / 2}=e^{-i s_{j} a} e^{-i s_{j} r_{j}^{2} / 2} .
$$

Using this identity in (4.15) and recalling the definition of $K$, we have proved (4.14). We now sum over $\mathbf{k}$ and $\mathbf{k}^{\prime}$ and apply the identity (4.14) for $a=\lambda^{2} \Theta\left(v_{0}\right)$ :

$$
\sum_{\substack{\mathbf{k}, \mathbf{k}^{\prime} \\|\mathbf{k}|=\left|\mathbf{k}^{\prime}\right|=m}} Y\left(\xi, \mathbf{v}, \mathbf{k}, \mathbf{k}^{\prime}\right)=\lambda^{2 m} e^{i t \lambda^{2}\left[\bar{\Theta}\left(v_{0}\right)-\Theta\left(v_{0}\right)\right]} \bar{K}\left(t ; \mathbf{v}-\frac{\xi}{2} ; m\right) K\left(t ; \mathbf{v}+\frac{\xi}{2} ; m\right)|M(\mathbf{v}, m)|^{2} \widehat{W}_{\psi_{0}}\left(\xi, v_{m}\right) .
$$

From (4.13) and (4.16), we have a new representation of $\sum_{\pi} C_{\pi}^{\varepsilon, \text { main }}$ :

$$
\begin{aligned}
\sum_{\substack{\pi:|\pi|=m \\
\pi \text { simple }}} C_{\pi}^{\varepsilon, \text { main }} & =\lambda^{2 m} e^{i t \lambda^{2}\left[\bar{\Theta}\left(v_{0}\right)-\Theta\left(v_{0}\right)\right]} \int d \xi \int d \mathbf{v}_{m} \varepsilon^{-d} \overline{\widehat{J}\left(\xi \varepsilon^{-1}, v_{0}\right)} \\
& \times \bar{K}\left(t ; \mathbf{v}-\frac{\xi}{2} ; m\right) K\left(t ; \mathbf{v}+\frac{\xi}{2} ; m\right)|M(\mathbf{v}, m)|^{2} \widehat{W}_{\psi_{0}}\left(\xi, v_{m}\right)
\end{aligned}
$$

By definition of $K$,

$$
\begin{gathered}
\overline{K\left(t ; \mathbf{v}-\frac{\xi}{2} ; m\right)} K\left(t ; \mathbf{v}+\frac{\xi}{2} ; m\right)=\int_{0}^{\infty} d s_{0} d s_{1} \ldots d s_{m} \delta\left(t-\sum_{j=0}^{m} s_{j}\right) \\
\times \int_{0}^{\infty} d s_{0}^{\prime} d s_{1}^{\prime} \ldots d s_{m}^{\prime} \delta\left(t-\sum_{j=0}^{m} s_{j}^{\prime}\right) \prod_{j=0}^{m} \exp \left\{\frac{i}{2}\left[s_{j}\left(v_{j}-\frac{\xi}{2}\right)^{2}-s_{j}^{\prime}\left(v_{j}+\frac{\xi}{2}\right)^{2}\right]\right\} .
\end{gathered}
$$

Let $a_{j}=\left(s_{j}+s_{j}^{\prime}\right) / 2, b_{j}=\left(s_{j}-s_{j}^{\prime}\right) / 2$. Thus

$$
\begin{aligned}
\overline{K\left(t ; \mathbf{v}-\frac{\xi}{2} ; m\right)} K\left(t ; \mathbf{v}+\frac{\xi}{2} ; m\right)= & 2^{m} \prod_{j=0}^{m} \int_{0}^{t} d a_{j} \delta\left(t-\sum_{j=0}^{m} a_{j}\right) e^{-i \xi \cdot \sum_{j=0}^{m} a_{j} v_{j}} \\
& \times \prod_{j=0}^{m} \int_{-a_{j}}^{a_{j}} d b_{j} \delta\left(\sum_{j=0}^{m} b_{j}\right) e^{i \sum_{j=0}^{m} b_{j} v_{j}^{2}} .
\end{aligned}
$$

The last integral can be rewritten as

$$
\int_{-\infty}^{\infty} d \mathbf{b} \chi_{\mathbf{a}}(\mathbf{b}) e^{i \sum_{j=0}^{m-1} b_{j}\left(v_{j}^{2}-v_{m}^{2}\right)}
$$

where

$$
\chi_{\mathbf{a}}(\mathbf{b}):=\chi\left(-a_{0} \leq \sum_{j=0}^{m-1} b_{j} \leq a_{0}\right) \prod_{j=0}^{m-1} \chi\left(-a_{j} \leq b_{j} \leq a_{j}\right)
$$


Here $\chi$ is the characteristic function and $\mathbf{b}=\left(b_{0}, b_{1}, b_{2}, \ldots b_{m-1}\right), \mathbf{a}=\left(a_{0}, a_{1}, \ldots a_{m}\right)$.

We change the variables to the macroscopic variables: $\alpha=\varepsilon a, \zeta=\varepsilon^{-1} \xi, t=\varepsilon^{-1} T$ with $\varepsilon=\lambda^{2}$. Notice that $i\left[\bar{\Theta}\left(v_{0}\right)-\Theta\left(v_{0}\right)\right]=2 \operatorname{Im} \Theta\left(v_{0}\right)$. We can now rewrite $\sum_{\pi} C_{\pi}^{\varepsilon, \text { main }}$ as

$$
\begin{aligned}
& \sum_{\substack{\pi:|\pi|=m \\
\pi \text { simple }}} C_{\pi}^{\varepsilon, \text { main }}=2^{m} \int d \mathbf{v}_{m}|M(\mathbf{v}, m)|^{2} e^{2 T \operatorname{Im} \Theta\left(v_{0}\right)}\left(\prod_{j=0}^{m} \int_{0}^{T} d \alpha_{j}\right) \delta\left(T-\sum_{j=0}^{m} \alpha_{j}\right) \\
& \times \int_{-\infty}^{\infty} d \mathbf{b} \chi_{\varepsilon^{-1}} \boldsymbol{\alpha}(\mathbf{b}) e^{i \sum_{j=0}^{m-1} b_{j}\left(v_{j}^{2}-v_{m}^{2}\right)} \int d \zeta \overline{\widehat{J}\left(\zeta, v_{0}\right)} e^{-i \zeta \cdot \sum_{j=0}^{m} \alpha_{j} v_{j}} \widehat{W}_{\psi_{0}}\left(\varepsilon \zeta, v_{m}\right) .
\end{aligned}
$$

Notice that the $\lambda^{2 m}$ factor in (4.16) is cancelled by $\varepsilon^{-m}$ from the change of variables. The last integral is the Fourier transform of a product and thus it is equal to

$$
\int d X \overline{J\left(X, v_{0}\right)} W_{\psi_{0}}^{\varepsilon}\left[\left(X-\sum_{j=0}^{m} \alpha_{j} v_{j}\right), v_{m}\right]=\int d Y \bar{J}\left(Y+\sum_{j=0}^{m} \alpha_{j} v_{j}, v_{0}\right) W_{\psi_{0}}^{\varepsilon}\left(Y, v_{m}\right) .
$$

From the definition of the initial data $\psi_{0}=\psi_{0}^{\varepsilon}$ in the main Theorem,

$$
W_{\psi_{0}^{\varepsilon}}^{\varepsilon}\left(Y, v_{m}\right)=\varepsilon^{-d} W_{\psi_{0}^{\varepsilon}}\left(Y / \varepsilon, v_{m}\right) \rightarrow F_{0}\left(Y, v_{m}\right)
$$

weakly as $\varepsilon \rightarrow 0$. For any fixed $m$ and $\boldsymbol{\alpha}$

$$
\int_{-\infty}^{\infty} d \mathbf{b} \chi_{\varepsilon^{-1} \alpha}(\mathbf{b}) \prod_{j=0}^{m-1} e^{i b_{j}\left(v_{j}^{2}-v_{m}^{2}\right)} \rightarrow \prod_{j=0}^{m-1}\left(2 \pi \delta\left(v_{j}^{2}-v_{m}^{2}\right)\right)
$$

weakly as $\varepsilon \rightarrow 0$. In particular

$$
\begin{gathered}
\lim _{\varepsilon \rightarrow 0} \int d v_{0} \ldots d v_{m-1}|M(\mathbf{v}, m)|^{2} \bar{J}\left(Y+\sum_{j=0}^{m} \alpha_{j} v_{j}, v_{0}\right) \int_{-\infty}^{\infty} d \mathbf{b} \chi_{\varepsilon^{-1} \alpha}(\mathbf{b}) \prod_{j=0}^{m-1} e^{i b_{j}\left(v_{j}^{2}-v_{m}^{2}\right)} \\
=\int d v_{0} \ldots d v_{m-1}|M(\mathbf{v}, m)|^{2} \bar{J}\left(Y+\sum_{j=0}^{m} \alpha_{j} v_{j}, v_{0}\right) \prod_{j=0}^{m-1}\left(2 \pi \delta\left(v_{j}^{2}-v_{m}^{2}\right)\right)
\end{gathered}
$$

since $J, R \in \mathcal{S}$. This convergence is in $\mathcal{S}\left(\mathbf{R}_{Y}^{d} \times \mathbf{R}_{v_{m}}^{d}\right)$. Hence we can multiply the limits (4.17) and (4.18).

After changing the variable $Y$ back to $X$ we have thus proved that

$$
\lim _{\varepsilon \rightarrow 0} \sum_{\substack{\pi:||||=m \\ \pi \text { simple }}} C_{\pi}^{\varepsilon, \text { main }}=\int d X d \mathbf{v}_{m} \overline{J\left(X, v_{0}\right)} e^{2 T \operatorname{Im} \Theta\left(v_{0}\right)}
$$




$$
\times\left(\prod_{j=0}^{m} \int_{0}^{T} d \alpha_{j}\right) \delta\left(T-\sum_{j=0}^{m} \alpha_{j}\right) \prod_{j=0}^{m-1} \sigma\left(v_{j}, v_{j-1}\right) F_{0}\left(X-\sum_{j=0}^{m} \alpha_{j} v_{j}, v_{m}\right)
$$

where

$$
\sigma(U, V):=4 \pi R^{2}(U-V) \delta\left(U^{2}-V^{2}\right) .
$$

Combining (4.1), (4.12) and (4.19) we obtain

$$
\begin{gathered}
\lim _{M \rightarrow \infty} \lim _{\varepsilon \rightarrow 0} \mathbf{E} \int \overline{\widehat{J}_{\varepsilon}(\xi, v)} \widehat{W}_{\psi_{T / \varepsilon, M}^{\text {main }}}(\xi, v) d \xi d v \\
=\sum_{m=0}^{\infty} \int d X \int d V \overline{J(X, V)} \int d V_{1} \ldots d V_{m} \int_{0} d \boldsymbol{\tau}_{m} \delta\left(T-\sum \tau_{j}\right) \\
\times e^{2 T I m \Theta(V)} \sigma\left(V, V_{1}\right) \sigma\left(V_{1}, V_{2}\right) \ldots \sigma\left(V_{m-1}, V_{m}\right) F_{0}\left(X-\sum_{j=0}^{m} \tau_{j} V_{j}, V_{m}\right),
\end{gathered}
$$

with $V=V_{0}$. Since this holds for all smooth functions $J$,

$$
\lim _{M \rightarrow \infty} \lim _{\varepsilon \rightarrow 0} \mathbf{E} W_{\psi_{T / \varepsilon, M}^{\operatorname{main}}}^{\varepsilon}(X, V)=F_{T}(X, V)
$$

weakly with

$$
\begin{aligned}
F_{T}(X, V):= & \sum_{m=0}^{\infty} e^{2 T \operatorname{Im} \Theta(V)} \int d V_{1} d V_{2} \ldots d V_{m} \int_{0} d \boldsymbol{\tau}_{m} \delta\left(T-\sum_{j=0}^{m} \tau_{j}\right) \\
& \times \sigma\left(V, V_{1}\right) \sigma\left(V_{1}, V_{2}\right) \ldots \sigma\left(V_{m-1}, V_{m}\right) F_{0}\left(X-\sum_{j=0}^{m} \tau_{j} V_{j}, V_{m}\right) .
\end{aligned}
$$

We can check that the function $F_{T}(X, V)$ satisfies the equation

$$
\partial_{T} F_{T}(X, V)+V \cdot \nabla_{X} F_{T}(X, V)=\int\left[\sigma(U, V) F_{T}(X, U)\right] d U+2 \operatorname{Im} \Theta(V) F_{T}(X, V) .
$$

Recall $\sigma(V)$ is the total differential cross section given by

$$
\sigma(V):=\int \sigma(U, V) d U
$$

By radial symmetry, $\sigma(V)=\sigma(|V|)$. Recall the definition of $\Theta$ from (3.59) and (3.74). It is easy to check that

$$
\operatorname{Im} \Theta(V)=-2 \pi \int_{\mathbf{R}^{d}} d q R^{2}(V-q) \delta\left(V^{2}-q^{2}\right)=-\frac{\sigma(V)}{2} .
$$

This is actually the second order equality of the optical theorem. From the last relation and the equation (4.21), we have proved that the Wigner transform of the main term converges weakly to the solution of the linear Boltzmann equation with the scattering kernel $\sigma$. 


\section{Partial Time Integration and Estimate of $\Psi_{n_{0}}(t)$.}

We shall estimate the $L^{2}$ norm of $\Psi_{n_{0}}(t)$ in this section (recall (2.1) for the definition). Our basic idea is to subdivide the time integration into smaller time intervals of size $t / \kappa(\varepsilon)$ with $\kappa(\varepsilon)$ to be chosen in (5.10). We then use the Duhamel formula to estimate the evolution in each time interval. In other words, instead of using the Duhamel formula to control directly the wave function $\Psi_{n_{0}}(t)$, we first subdivide the time interval and then use the Duhamel formula in each time interval. We shall see that this procedure improves the error estimates.

We now define several time dependent wave functions below. The wave functions have two variables: $\left(t, p_{0}\right)$ for the time and momentum. We shall omit one or both variables if they are not relevant in the formulas. The choice of $\kappa=\kappa(\varepsilon)$ and $n_{0}=n_{0}(\varepsilon)$ depend on $\varepsilon$, but we will omit this argument in most cases. Let $\theta_{j}=j t / \kappa$ for $j=0,1, \ldots \kappa$. Rewrite $\Psi_{n_{0}}(t)$ in $(2.1)$ as

$$
\Psi_{n_{0}}(t)=(-i) \int_{0}^{t} d s e^{-i(t-s) H} \widetilde{\psi}_{n_{0}}(s)=(-i) \sum_{j=0}^{\kappa-1} e^{-i\left(t-\theta_{j+1}\right) H} \int_{\theta_{j}}^{\theta_{j+1}} e^{-i\left(\theta_{j+1}-s\right) H} \widetilde{\psi}_{n_{0}}(s) d s .
$$

We now use the Duhamel formula to expand the unitary group $e^{-i\left(\theta_{j+1}-s\right) H}$. Recall the definition of $L$ in (2.5). Define a kernel

$$
\widetilde{D}(t ; \mathbf{p}, m):=K(t ; \mathbf{p}, m-1) L(\mathbf{p}, m)
$$

and the corresponding operator

$$
\left(\widetilde{\mathcal{D}}_{t}(m) \widehat{\psi}\right)\left(p_{0}\right):=\int d \mathbf{p}_{m, \widehat{0}} \widetilde{D}(t ; \mathbf{p}, m) \widehat{\psi}\left(p_{m}\right) .
$$

For $n \geq m$ and $s>\theta$, define the wavefunction with the $(m+1)$-th collision after time $\theta$ and $n$ total collisions up to time $s$ by

$$
\widehat{\psi}_{n, m, \theta}(s)=\int_{\theta}^{s} d \sigma \widetilde{D}_{s-\sigma}(n-m) \widehat{\psi}_{m}(\sigma) .
$$

We also define the amputated versions of these functions in momentum space: for $n>m$

$$
\widetilde{\psi}_{n, m, \theta}\left(s, p_{0}\right):=\lambda \int d p \widehat{V}\left(p_{0}-p\right) \widehat{\psi}_{n-1, m, \theta}(s, p) .
$$

The Duhamel formula for $e^{-i\left(\theta_{j+1}-s\right) H}$ gives

$$
\Psi_{n_{0}}=U_{1}+U_{2}
$$


where

$$
\begin{gathered}
U_{1}(t)=\sum_{n_{0} \leq n<4 n_{0}} \sum_{j=0}^{\kappa-1} e^{-i\left(t-\theta_{j+1}\right) H} \psi_{n, n_{0}, \theta_{j}}\left(\theta_{j+1}\right), \\
U_{2}(t)=(-i) \sum_{j=0}^{\kappa-1} e^{-i\left(t-\theta_{j+1}\right) H} \int_{\theta_{j}}^{\theta_{j+1}} e^{-i\left(\theta_{j+1}-s\right) H} \widetilde{\psi}_{4 n_{0}, n_{0}, \theta_{j}}(s) d s .
\end{gathered}
$$

From the unitarity of $e^{-i\left(t-\theta_{j+1}\right) H}$ and the triangle inequality, we can bound $U_{1}$ by

$$
\left\|U_{1}\right\|_{2}^{2} \leq C n_{0} \kappa \sum_{n_{0} \leq n<4 n_{0}} \sum_{j=0}^{\kappa-1}\left\|\psi_{n, n_{0}, \theta_{j}}\left(\theta_{j+1}\right)\right\|_{2}^{2}
$$

This is a discrete version of the following inequality based on the unitarity and triangle inequality. Its proof is obvious.

Lemma 5.1 . For any function $h_{s}$ we have

$$
\left\|\int_{0}^{t} e^{-i(t-s) H} h_{s} d s\right\|_{2}^{2} \leq t \int_{0}^{t}\left\|h_{s}\right\|_{2}^{2} d s .
$$

From this Lemma, we can bound $U_{2}$ by

$$
\left\|U_{2}(t)\right\|_{2}^{2} \leq t \sum_{j=0}^{\kappa-1} \int_{\theta_{j}}^{\theta_{j+1}}\left\|\widetilde{\psi}_{4 n_{0}, n_{0}, \theta_{j}}(s)\right\|_{2}^{2} d s \leq t^{2} \sup _{j} \sup _{\theta_{j} \leq s \leq \theta_{j+1}}\left\|\widetilde{\psi}_{4 n_{0}, n_{0}, \theta_{j}}(s)\right\|_{2}^{2}
$$

The $L^{2}$ norms $\left\|\psi_{n, n_{0}, \theta_{j}}\left(\theta_{j+1}\right)\right\|_{2}^{2}$ and $\left\|\widetilde{\psi}_{4 n_{0}, n_{0}, \theta_{j}}(s)\right\|_{2}^{2}$ in the last two equations are estimated in the following Lemma which we use for $\tau=\theta_{j+1}$ and $\tau=s$.

Lemma 5.2. For $0 \leq \tau-\theta \leq t / \kappa, \theta \leq t-t / \kappa, \kappa \geq 2, n_{0} \leq n$ and $0 \leq a<1$ we have

$$
\mathbf{E}\left\|\psi_{n, n_{0}, \theta}(\tau)\right\|^{2} \leq \frac{\left(C \lambda^{2} t\right)^{n}}{(n !)^{1 / 2}}\left[(\log t)^{4}+\frac{(n !)^{3 / 2}\left(C_{a} \log t\right)^{n+5}}{t^{a / 2}}\right]
$$

(we will use this for $n_{0} \leq n<4 n_{0}$ ). For $3 n_{0}+2 \leq n$ (we use it for $n=4 n_{0}$ )

$$
\mathbf{E}\left\|\psi_{n, n_{0}, \theta}(\tau)\right\|^{2} \leq \frac{n !\left(C \lambda^{2} t\right)^{n}(\log t)^{n+4}}{\kappa^{n_{0}}}
$$

and

$$
\mathbf{E}\left\|\widetilde{\psi}_{n, n_{0}, \theta}(\tau)\right\|^{2} \leq \frac{n !\left(C \lambda^{2} t\right)^{n}(\log t)^{n+4}}{\kappa^{n_{0}}}
$$

Remark: These estimates are not optimal. The true exponent of $\kappa$ in (5.7), instead of $n_{0}$, is $n-n_{0}$ corresponding to $n-n_{0}$ collisions in a time interval of length $t / \kappa$. In the last estimate 
(5.8), we can get an extra $\frac{1}{t}$ due to the definition of $\widetilde{\psi}$ starting with a potential. But we do not need these stronger results.

We now use this Lemma to bound $U_{1}$ and $U_{2}$. Recall the scaling of coupling constant $\lambda=\sqrt{\varepsilon}$ and $t=T / \varepsilon$. Notice that $\lambda^{2} t$ is bounded by $T$, independent of $\varepsilon$. Hence we can bound $U_{1}$ and $U_{2}$ by

$$
\begin{aligned}
\left\|U_{1}(t)\right\|_{2}^{2} & \leq \frac{C^{n_{0}} n_{0}^{2} \kappa^{2}|\log \varepsilon|^{4}}{\sqrt{n_{0} !}}+C_{a}^{n_{0}} n_{0}^{2} \kappa^{2}|\log \varepsilon|^{4 n_{0}+5} \varepsilon^{\frac{a}{2}}\left(4 n_{0}\right) ! \\
\left\|U_{2}(t)\right\|_{2}^{2} & \leq \frac{\left(4 n_{0}\right) ! C^{n_{0}}|\log \varepsilon|^{4 n_{0}+4}}{\varepsilon^{2} \kappa^{n_{0}}} .
\end{aligned}
$$

Though these estimates look complicated, they are from a few simple observations. The estimate on $U_{1}$ has two terms: the first one is from the simple pairings; the second from the non-simple pairings. The last term for the non-simple pairings has the factor $\left(4 n_{0}\right)$ ! from the number of pairings and $\varepsilon^{a / 2}$ from the phase cancellation of the non-simple pairings. The first term from the simple pairings has neither $\left(4 n_{0}\right)$ ! nor the factor $\varepsilon^{a / 2}$. It gains at least a factor $\sqrt{n_{0}}$ ! in the denominator from the time integration. The true size of this factor should be $n_{0}$ ! and we lost half of it in the estimate. Since we use the unitarity in estimating $U_{1}$, we in principle lose a factor $t$. But $U_{1}$ is fully expanded in a subinterval of length $t / \kappa$. This reduces the factor from $t$ to $\kappa^{2}$. In the estimate of $U_{2}$, the key factor is the denominator $\kappa^{n_{0}}$, coming from putting $3 n_{0}$ collisions in a time interval $t / \kappa$. The true size of this factor is $\kappa^{3 n_{0}}$, which we obtain only partially.

We choose

$$
n_{0}=n_{0}(\varepsilon):=\frac{\gamma|\log \varepsilon|}{\log |\log \varepsilon|}, \quad \kappa=\kappa(\varepsilon):=|\log \varepsilon|^{1 / \gamma^{2}}
$$

with a sufficiently small positive $\gamma \ll 1$. Notice this choice gives

$$
\lim _{\varepsilon \rightarrow 0} \varepsilon^{\alpha}|\log \varepsilon|^{n_{0}(\varepsilon)}=0, \quad \lim _{\varepsilon \rightarrow 0} \varepsilon^{\alpha}\left[n_{0}(\varepsilon)\right] !=0, \quad \lim _{\varepsilon \rightarrow 0} \varepsilon^{\beta} \kappa(\varepsilon)^{n_{0}(\varepsilon)}=\infty
$$

for any $\alpha>\gamma$ and $\beta<\gamma^{-1}$. Thus the three terms on the right hand sides of (5.9) vanish in the limit $\varepsilon \rightarrow 0$. The rest of this section is devoted to prove Lemma 5.2.

Proof of Lemma 5.2. Denote

$$
I_{n-n_{0}, n}:=\left\{n-n_{0}, n-n_{0}+1, \ldots, n\right\} .
$$

Define the free evolution operator with constraint given by the parameters $n_{0}, \theta$ as

$$
K^{\#}\left(t, \theta ; \mathbf{p}, n, n_{0}\right):=\int_{\theta}^{t} K\left(t-s, \mathbf{p}, n-n_{0}-1\right) K\left(s, \mathbf{p}, I_{n-n_{0}, n}\right) d s .
$$


The sign \# refers to the partial time constraint. We can rewrite $\psi_{n, n_{0}, \theta}(\tau)$ as

$$
\widehat{\psi}_{n, n_{0}, \theta}\left(\tau, p_{0}\right)=\int K^{\#}\left(\tau, \theta ; \mathbf{p}, n, n_{0}\right) L(\mathbf{p}, n) \widehat{\psi}_{0}\left(p_{n}\right) d \mathbf{p}_{n, \widehat{0}}
$$

The pairing structure in the Gaussian expectation $\mathbf{E}\left\|\psi_{n, n_{0}, \theta}(\tau)\right\|^{2}$ depends only on the potential part and is independent of the $K$-kernels. Thus the generated deltafunctions are given by $\Pi_{n, n}$ from Section 3 and are independent of $n_{0}$. Hence we can write (cf. (2.17))

$$
\begin{aligned}
\mathbf{E}\left\|\psi_{n, n_{0}, \theta}(\tau)\right\|^{2} & =\sum_{\pi \in \Pi_{n, n}} C_{\pi}^{\#} \\
C_{\pi}^{\#}: & : \lambda^{2 n} \int d \mathbf{p}_{n} d \mathbf{p}_{n}^{\prime} \overline{K^{\#}\left(\tau, \theta ; \mathbf{p}, n, n_{0}\right)} K^{\#}\left(\tau, \theta ; \mathbf{p}^{\prime}, n, n_{0}\right) \Delta_{\pi}^{0}\left(\mathbf{p}, \mathbf{p}^{\prime}\right) \overline{F(\mathbf{p}, n)} F\left(\mathbf{p}^{\prime}, n\right) .
\end{aligned}
$$

For the amputated function we just omit $p_{0}$ from the argument of $K^{\#}$ :

$$
\begin{aligned}
\mathbf{E}\left\|\widetilde{\psi}_{n, n_{0}, \theta}(\tau)\right\|^{2} & =\sum_{\pi} \widetilde{C}_{\pi}^{\#} \\
\widetilde{C}_{\pi}^{\#}: & :=\lambda^{2 n} \int d \mathbf{p}_{n} d \mathbf{p}_{n}^{\prime} \overline{\widetilde{K}^{\#}\left(\tau, \theta ; \mathbf{p}, n, n_{0}\right)} \widetilde{K}^{\#}\left(\tau, \theta ; \mathbf{p}^{\prime}, n, n_{0}\right) \Delta_{\pi}^{0}\left(\mathbf{p}, \mathbf{p}^{\prime}\right) \overline{F(\mathbf{p}, n)} F\left(\mathbf{p}^{\prime}, n\right)
\end{aligned}
$$

where

$$
\widetilde{K}^{\#}\left(\tau, \theta ; \mathbf{p}, n, n_{0}\right):=\int_{\theta}^{\tau} K\left(\tau-s, \mathbf{p}, I_{1, n-n_{0}-1}\right) K\left(s, \mathbf{p}, I_{n-n_{0}, n}\right) d s .
$$

Since the number of simple pairings is at most $C^{n}$, Lemma 5.2 follows from Lemmas 5.3, 5.4, 5.5 and 5.6 below.

We first prove the following apriori estimate. This implies in particular the estimates (5.7) and (5.8) of Lemma 5.2. The estimate obtained in the following Lemma is not optimal and the remark after Lemma 5.2 applies as well.

Lemma 5.3 . For any $\pi \in \Pi_{n, n}$ we have for $n \geq n_{0}$

$$
\left|C_{\pi}^{\#}\right| \leq\left(C \lambda^{2} t\right)^{n}(\log t)^{n+4}
$$

and for $n \geq 3 n_{0}+2$

$$
\begin{aligned}
& \left|C_{\pi}^{\#}\right| \leq \frac{\left(C \lambda^{2} t\right)^{n}(\log t)^{n+4}}{\kappa^{n_{0}}} \\
& \left|\widetilde{C}_{\pi}^{\#}\right| \leq \frac{\left(C \lambda^{2} t\right)^{n}(\log t)^{n+4}}{\kappa^{n_{0}}}
\end{aligned}
$$


Proof of Lemma 5.3. Recall the K-identity in Lemma 3.1. We can extend it to the following identity for $K^{\#}\left(\tau, \theta ; \mathbf{p}, n, n_{0}\right)$. Notice that we now need two integrations for two time constraints:

$$
\begin{aligned}
K^{\#}\left(\tau, \theta ; \mathbf{p}, n, n_{0}\right)=-\int_{\theta}^{\tau} d s & e^{(\tau-s) \eta} e^{s \tilde{\eta}} \int_{-\infty}^{\infty} d \alpha d \widetilde{\alpha} e^{-i \alpha(\tau-s)} e^{-i \widetilde{\alpha} s} \\
& \times \prod_{j=0}^{n-n_{0}-1} \frac{1}{\alpha-p_{j}^{2} / 2+i \eta} \prod_{j \in I_{n-n_{0}, n}} \frac{1}{\widetilde{\alpha}-p_{j}^{2} / 2+i \widetilde{\eta}},
\end{aligned}
$$

where we choose $\eta:=(\tau-\theta)^{-1}, \widetilde{\eta}:=t^{-1}$. We can integrate $s$ to have

$$
\begin{aligned}
K^{\#}\left(\tau, \theta ; \mathbf{p}, n, n_{0}\right)= & i \int_{-\infty}^{\infty} d \alpha d \widetilde{\alpha} \frac{e^{-i \tau \widetilde{\alpha}+\tau \widetilde{\eta}}-e^{-i \alpha(\tau-\theta)-i \theta \widetilde{\alpha}+(\tau-\theta) \eta+\theta \widetilde{\eta}}}{\alpha-\widetilde{\alpha}+i(\eta-\widetilde{\eta})} \\
& \times \prod_{j=0}^{n-n_{0}-1} \frac{1}{\alpha-p_{j}^{2} / 2+i \eta} \prod_{j \in I_{n-n_{0}, n}} \frac{1}{\widetilde{\alpha}-p_{j}^{2} / 2+i \widetilde{\eta}} .
\end{aligned}
$$

This yields the following estimate using that $\tau \widetilde{\eta} \leq 1,(\tau-\theta) \eta \leq 1, \theta \widetilde{\eta} \leq 1$ :

$$
\begin{aligned}
& \left|K^{\#}\left(\tau, \theta ; \mathbf{p}, n, n_{0}\right)\right| \\
& \quad \leq \int_{-\infty}^{\infty} d \alpha d \widetilde{\alpha} \frac{1}{|\alpha-\widetilde{\alpha}+i(\eta-\widetilde{\eta})|} \prod_{j=0}^{n-n_{0}-1} \frac{1}{\left|\alpha-p_{j}^{2} / 2+i \eta\right|} \prod_{j \in I_{n-n_{0}, n}} \frac{1}{\left|\widetilde{\alpha}-p_{j}^{2} / 2+i \widetilde{\eta}\right|} .
\end{aligned}
$$

Define

$$
\begin{gathered}
\alpha_{k}:= \begin{cases}\widetilde{\alpha} & \text { if } k \leq n-n_{0}-1 \\
\alpha & \text { if } n-n_{0} \leq k \leq n \\
\beta & \text { if } n+1 \leq k \leq n+n_{0}+1 \\
\widetilde{\beta} & \text { if } n+n_{0}+2 \leq k\end{cases} \\
\eta_{k}:= \begin{cases}\widetilde{\eta} & \text { if } k \leq n-n_{0}-1 \\
\eta & \text { if } n-n_{0} \leq k \leq n \\
\eta & \text { if } n+1 \leq k \leq n+n_{0}+1 \\
\widetilde{\eta} & \text { if } n+n_{0}+2 \leq k .\end{cases}
\end{gathered}
$$

Hence we can bound $C_{\pi}^{\#}$ by

$$
\begin{aligned}
\left|C_{\pi}^{\#}\right| \leq \lambda^{2 n} & \int d \mathbf{w}_{A} \int_{-\infty}^{\infty} d \alpha d \widetilde{\alpha} d \beta d \widetilde{\beta} \frac{1}{|\alpha-\widetilde{\alpha}+i(\eta-\widetilde{\eta})|} \frac{1}{|\beta-\widetilde{\beta}+i(\eta-\widetilde{\eta})|} \\
& \times \prod_{0 \leq k \leq 2 n} \frac{1}{\left|\alpha_{k}-w_{k}^{2} / 2+i \eta_{k}\right|}|F(\mathbf{p}, n)|\left|F\left(\mathbf{p}^{\prime}, n\right)\right| .
\end{aligned}
$$


If $j \in B$, we consider $w_{j}$ as a function of $\mathbf{w}_{A}$ given by (2.19). Thus we can estimate $\left|C_{\pi}^{\#}\right|$ by the following bound similar to (3.31):

$$
\begin{aligned}
\left|C_{\pi}^{\#}\right| \leq & \lambda^{2 n} \int_{-\infty}^{\infty} d \alpha d \widetilde{\alpha} d \beta d \widetilde{\beta} \frac{1}{|\alpha-\widetilde{\alpha}+i(\eta-\widetilde{\eta})||\beta-\widetilde{\beta}+i(\eta-\widetilde{\eta})|} \\
& \times \int d \mu\left(\mathbf{w}_{A}\right) \frac{1}{\left|\alpha-w_{0}^{2} / 2+i \eta\right|\left|\beta-w_{0}^{2} / 2+i \eta\right|} \prod_{\substack{\ell \in A \\
\ell \neq 0}} \frac{1}{\left|\alpha_{\ell}-w_{\ell}^{2} / 2+i \eta_{\ell}\right|} \\
& \times \prod_{\substack{j \in B \\
j \neq 2 n+1}} \frac{1}{\left|\alpha_{j}-w_{j}^{2} / 2+i \eta_{j}\right|}
\end{aligned}
$$

Notice that $\eta, \widetilde{\eta} \geq t^{-1}$ and $|\eta-\widetilde{\eta}| \geq t^{-1}$ (since $\tau-\theta \leq \frac{t}{\kappa} \leq \frac{t}{2}$ ). Following the proof of Lemma 3.4 , we can bound $\left|C_{\pi}^{\#}\right|$ by

$$
\left|C_{\pi}^{\#}\right| \leq\left(C \lambda^{2} t\right)^{n}(\log t)^{n+4}
$$

We now prove the stronger estimate (5.13) for $n \geq 3 n_{0}+2$. Notice that we can bound the last product in (5.17) by

$$
\prod_{\substack{j \in B \\ j \neq 2 n+1}} \frac{1}{\left|\alpha_{j}-w_{j}^{2} / 2+i \eta_{j}\right|} \leq \prod_{\substack{j \in B \\ j \neq 2 n+1}}\left|\eta_{j}\right|^{-1} .
$$

From the definition of $B$ in (2.19), we have $|B|=n+1$. The total number of $\widetilde{\eta}$ 's among $\eta_{1}, \eta_{2}, \ldots \eta_{2 n}$ is $2 n_{0}+2$. Hence among the $\eta_{k}$ in $B$, there are at least $n-2 n_{0}-2\left(\geq n_{0}\right)$ of them with $\eta_{k}=(\tau-\theta)^{-1}$. Hence

$$
\prod_{\substack{j \in B \\ j \neq 2 n+1}}\left|\eta_{j}\right|^{-1} \leq t^{n}\left(\frac{\tau-\theta}{t}\right)^{n_{0}} \leq \frac{t^{n}}{\kappa^{n_{0}}} .
$$

This proves (5.13).

Finally, to obtain (5.14), we just notice that the factor $\left|\alpha-w_{0}^{2} / 2+i \eta\right|^{-1}\left|\beta-w_{0}^{2} / 2+i \eta\right|^{-1}$ is not present in the formula for $\widetilde{C}_{\pi}^{\#}$ similar to $(5.17)$. The rest of the proof is the same. If we keep track of this omission carefully, then there is one less time integration and we obtain an extra $t^{-1}$ in the estimate of $\widetilde{\psi}_{n, n_{0}, \theta}(t)$. But we do not need it here. This finishes the proof of Lemma 5.3.

Lemma 5.4. If $\pi$ is crossing then

$$
\left|C_{\pi}^{\#}\right| \leq\left(C \lambda^{2} t\right)^{n} t^{-1}(\log t)^{n+5}
$$

Proof of Lemma 5.4. The proof is essentially the same as in Lemma 2.4. We only have to use $\eta, \widetilde{\eta} \geq t^{-1}$ and $|\eta-\widetilde{\eta}| \geq t^{-1}$. We shall not repeat the argument here. 
Lemma 5.5 . For any nested $\pi \in \Pi_{n, n}$, the following bound holds:

$$
\left|C_{\pi}^{\#}\right| \leq\left(C \lambda^{2} t\right)^{n} t^{-1}(\log t)^{n+4}
$$

Proof of Lemma 5.5. Recall from the proof of Lemma 3.6 that a nest consists of an inner and an outer delta function: $\delta\left(-p_{i}+p_{i+1}-p_{j}+p_{j+1}\right)$ and $\delta\left(-p_{k}+p_{k+1}-p_{u}+p_{u+1}\right), i<k<u<j$, and we can assume that the inner delta function is minimal: $u=k+1$. There can be several inner deltas belonging to the same outer delta. We can consider a nest where all the inner deltas are minimal. Such a nest is called minimal nest. Recall the time threshold $s$ in (5.11). If the outer delta is entirely before the time $s$ or entirely after $s$, we can follow the proof of Lemma 3.6. Instead of using the apriori estimate Lemma 3.4 in the proof of Lemma 3.6, we now use Lemma 5.3. We thus obtain the estimate $\left(C \lambda^{2} t\right)^{n} t^{-1}(\log t)^{n+3}$.

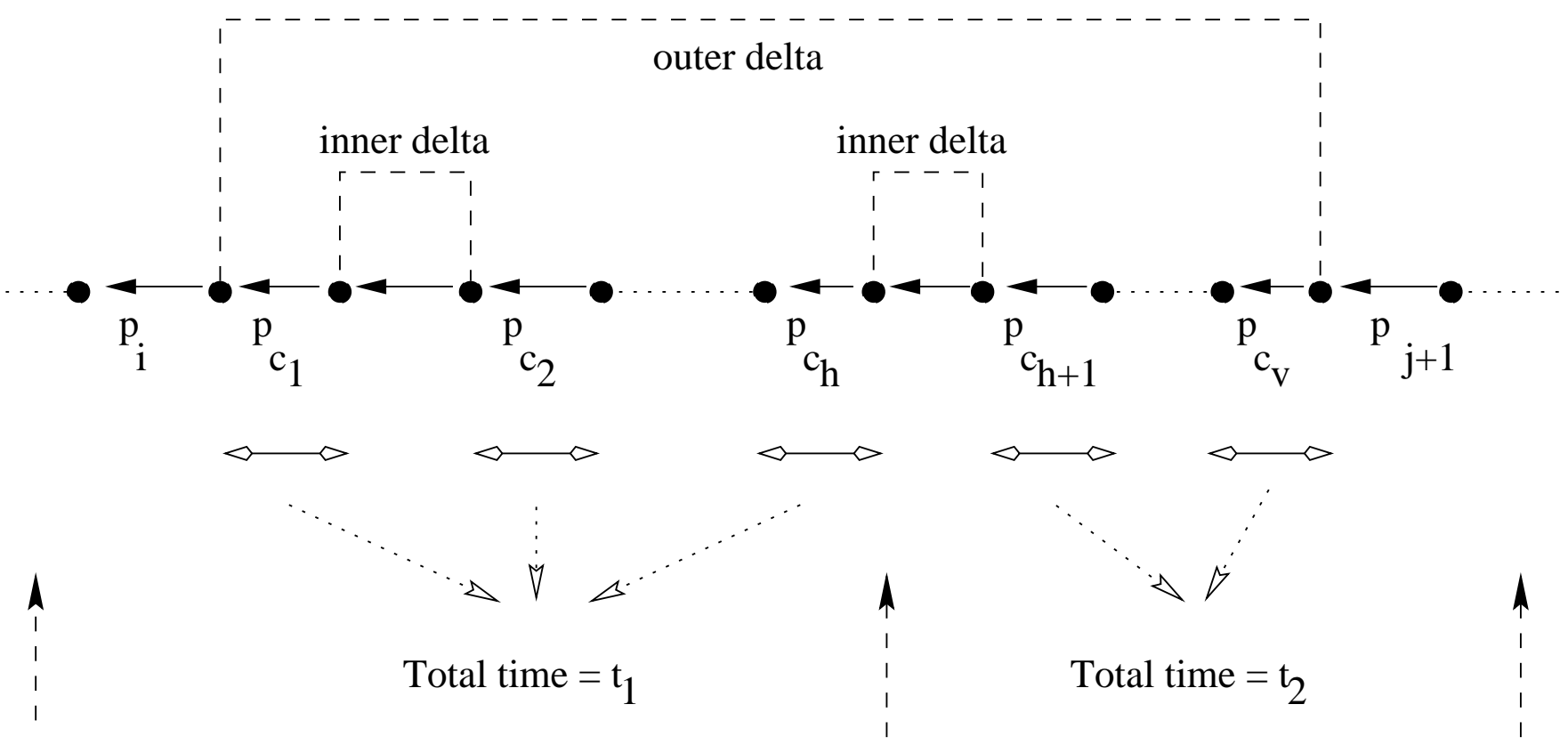

Time $=\tau$

Time $=\mathrm{s}>\theta$

Time $=0$

Fig. 4. Case $\mathrm{c}_{\mathrm{h}}=\mathrm{n}-\mathrm{n}_{0}-1$

The only case left to consider is when the outer delta of a minimal nest bridges over the time constraint $s$, i.e. if $i \leq n-n_{0}-1$ but $j \geq n-n_{0}$. Recall that we have $u=k+1$ for all inner delta function $\delta\left(-p_{k}+p_{k+1}-p_{u}+p_{u+1}\right)$ (as in Fig. 2). Hence by Lemma 2.6, $p_{k}=p_{k+2}$. Let $p_{c_{1}}, p_{c_{2}}, \ldots p_{c_{v}}$ be those momenta with $i+1=c_{1}<c_{2}<\ldots<c_{v}=j$ which 
are the same as $p_{k}=p_{k+2}$, i.e.

$$
p_{c_{1}}=p_{c_{2}}=\ldots=p_{k}=p_{k+2}=\ldots=p_{c_{v}} \text {. }
$$

Recall $I^{*}:=I_{n} \backslash\left\{c_{1}, \ldots, c_{v}\right\}$ and $\widetilde{\Delta}_{\pi}^{0}\left(\mathbf{p}^{*}, \mathbf{p}\right)$ (see (3.42)). Suppose that $p_{c_{h}}$ is the first momentum after time $s$, i.e. $1 \leq h \leq v-1$ is defined by $c_{h} \leq n-n_{0}-1<n-n_{0} \leq c_{h+1}$. Recall the identity (3.43). We apply this identity to the two $K$ kernels defining $K^{\#}$ (see (5.11)) with $q$ replaced by $p_{k}$. Thus we obtain

$$
\begin{aligned}
K^{\#}\left(\tau, \theta ; \mathbf{p}, n, n_{0}\right)=\int_{\theta}^{\tau} & d s \int_{0}^{\tau-s} d t_{1} K\left(\tau-s-t_{1}, \mathbf{p}, I^{*} \cap I_{n-n_{0}-1}\right) \frac{t_{1}^{h-1}}{(h-1) !} e^{-i t_{1} p_{k}^{2} / 2} \\
& \times \int_{0}^{s} d t_{2} K\left(s-t_{2}, \mathbf{p}, I^{*} \cap I_{n-n_{0}, n}\right) \frac{t_{2}^{v-h-1}}{(v-h-1) !} e^{-i t_{2} p_{k}^{2} / 2} .
\end{aligned}
$$

Recall the definition of $E\left(\mathbf{p}^{*}, \mathbf{p}^{\prime}, p_{k}\right)(3.44)$. We thus have the identity

$$
\begin{aligned}
C_{\pi}^{\#}= & \lambda^{2 n} \int d \mathbf{p}^{*} d p_{k} d \mathbf{p}_{n}^{\prime} K^{\#}\left(\tau, \theta ; \mathbf{p}^{\prime}, n, n_{0}\right) \widetilde{\Delta}_{\pi}^{0}\left(\mathbf{p}^{*}, \mathbf{p}^{\prime}\right) E\left(\mathbf{p}^{a} *, \mathbf{p}^{\prime}, p_{k}\right) \\
& \times \int_{\theta}^{\tau} d s \int_{0}^{\tau-s} d t_{1} K\left(\tau-s-t_{1}, \mathbf{p}, I^{*} \cap I_{n-n_{0}-1}\right) \frac{t_{1}^{h-1}}{(h-1) !} e^{-i t_{1} p_{k}^{2} / 2} \\
& \times \int_{0}^{s} d t_{2} K\left(s-t_{2}, \mathbf{p}, I^{*} \cap I_{n-n_{0}, n}\right) \frac{t_{2}^{v-h-1}}{(v-h-1) !} e^{-i t_{2} p_{k}^{2} / 2}
\end{aligned}
$$

Following the proof of Lemma 3.6, we integrate $p_{k}$ by parts. We thus obtain an identity for $C_{\pi}^{\#}$ in terms of $H\left(\mathbf{p}^{*}, \mathbf{p}^{\prime}, p_{k}\right)$ (see $\left.(3.45)\right)$, like $(3.46)$, but with an extra factor $\left(t_{1}+t_{2}\right)^{-1}$. Rewrite this factor by the simple identity

$$
\frac{1}{t_{1}+t_{2}}=\int_{0}^{\infty} e^{-\left(t_{1}+t_{2}\right) \mu} d \mu .
$$

We obtain the following expression of $C_{\pi}^{\#}$ :

$$
\begin{aligned}
C_{\pi}^{\#}= & \lambda^{2 n} \int d \mathbf{p}^{*} d p_{k} d \mathbf{p}_{n}^{\prime} \int_{0}^{\infty} d \mu K^{\#}\left(\tau, \theta ; \mathbf{p}^{\prime}, n, n_{0}\right) \widetilde{\Delta}_{\pi}^{0}\left(\mathbf{p}^{*}, \mathbf{p}^{\prime}\right) H\left(\mathbf{p}^{*}, \mathbf{p}^{\prime}, p_{k}\right) \\
& \times \int_{\theta}^{\tau} d s \int_{0}^{\tau-s} d t_{1} K\left(\tau-s-t_{1}, \mathbf{p}, I^{*} \cap I_{n-n_{0}-1}\right) \frac{t_{1}^{h-1}}{(h-1) !} e^{-i t_{1}\left(p_{k}^{2} / 2-i \mu\right)} \\
& \times \int_{0}^{s} d t_{2} K\left(s-t_{2}, \mathbf{p}, I^{*} \cap I_{n-n_{0}, n}\right) \frac{t_{2}^{v-h-1}}{(v-h-1) !} e^{-i t_{2}\left(p_{k}^{2} / 2-i \mu\right)} .
\end{aligned}
$$

We can perform the $d t_{1} d t_{2}$ integrations so that the last two lines are equal to

$$
\begin{aligned}
\int_{\theta}^{\tau} d s \overline{K^{*}\left(\tau-s, \mathbf{p}_{I^{*}}^{2}\right.} & \\
& \times \overline{K_{n-n_{0}-1}, \underbrace{p_{k}^{2}-2 i \mu, \ldots, p_{k}^{2}-2 i \mu}_{h \text { times }})} \\
& \mathbf{p}_{I^{*} \cap I_{n-n_{0}, n}}^{2} \underbrace{p_{k}^{2}-2 i \mu, \ldots, p_{k}^{2}-2 i \mu}_{v-h \text { times }})
\end{aligned}
$$


where $K^{*}$ is defined in (3.10) and $\mathbf{p}_{I^{*} \cap I_{n-n_{0}-1}}=\left\{p_{j}: j \in I^{*}, j \in I_{n-n_{0}-1}\right\}$. If the lower bound $\theta$ in the $s$ integration were replaced by 0 , we could use the semigroup identity of $K$ in (3.53) to obtain once more $K^{*}$, i.e., it is equal to

$$
\overline{K^{*}(\tau, \mathbf{p}_{I^{*} \cap I_{n-n_{0}-1}}^{2}, \underbrace{p_{k}^{2}-2 i \mu, \ldots, p_{k}^{2}-2 i \mu}_{v \text { times }})} .
$$

The lower bound $\theta$ complicates slightly the estimate.

We can now follow the proof of the apriori estimates Lemma 3.4 or Lemma 5.3 to bound $\left|C_{\pi}^{\#}\right|$ by

$$
\begin{gathered}
\left|C_{\pi}^{\#}\right| \leq \lambda^{2 n} \int_{-\infty}^{\infty} d \mu \int d \mu\left(\mathbf{w}_{A}\right) \int_{0}^{\infty} d \alpha d \widetilde{\alpha} d \beta d \widetilde{\beta} \frac{1}{|\alpha-\widetilde{\alpha}+i(\eta-\widetilde{\eta})|} \frac{1}{|\beta-\widetilde{\beta}+i(\eta-\widetilde{\eta})|} \\
\times H\left(\mathbf{p}^{*}, \mathbf{p}^{\prime}, p_{k}\right)\left|\frac{1}{\alpha-p_{k}^{2} / 2+i(\eta+\mu)}\right|^{h}\left|\frac{1}{\widetilde{\alpha}-p_{k}^{2} / 2+i(\widetilde{\eta}+\mu)}\right|^{v-h} \prod_{\substack{0 \leq k \leq 2 n \\
k \neq c_{j}, 1 \leq j \leq v}} \frac{1}{\left|\alpha_{k}-w_{k}^{2} / 2+i \eta_{k}\right|} .
\end{gathered}
$$

Here $\eta:=(\tau-\theta)^{-1}$ and $\widetilde{\eta}:=t^{-1}$. The definition of $\alpha_{k}$ and $\eta_{k}$ are the same as in the proof of Lemma 5.3. For each $\mu$ fixed, we can estimate the right hand side of the last inequality as in Lemma 5.3. The only difference is the regularization of $p_{k}^{2}$ by $i(\mu+\eta)$ instead of $i \eta$. The dependence on $\mu$ is rather easy to track. Integrating this $\mu$ dependent estimate over $\mu$, we have

$$
\left|C_{\pi}^{\#}\right| \leq\left(C \lambda^{2} t\right)^{n} t^{-1}(\log t)^{n+4} .
$$

The proves the Lemma 5.5.

Lemma 5.6 . For any simple $\pi \in \Pi_{n, n}$

$$
\left|C_{\pi}^{\#}\right| \leq \frac{\left(C \lambda^{2} t\right)^{n}}{(n !)^{1 / 2}}(\log t)^{4}+O\left(\left(C_{a} \lambda^{2} t\right)^{n} t^{-\frac{a}{2}}(\log t)^{n+2}\right)
$$

with $0 \leq a<1$.

The proof of Lemma 5.6 is similar to that of Lemma 3.7. Instead of the K-identity (3.7), we now use the representation (5.15) for $K^{\#}$. This gives a representation of the free evolution with time constraint. The rest of the proof for Lemma 5.6 is similar to Lemma 3.7. 


\section{The two dimensional case.}

We now prove Theorem 1.1 for $d=2$. This requires to extend all the arguments in section 3, 4 and 5 to $d=2$. The key Lemmas in these sections are Lemmas 3.5-3.12, Lemmas 4.2, 4.3 and Lemma 5.2. Since the proofs in Sect. 4 and 5 are taken from arguments in Sect. 3, once we extend the proofs in Sect. 3 to $d=2$, the modifications needed in Sect. 4 and 5 are obvious and will not repeated here. So we shall only prove Lemmas 3.5-3.12 for $d=2$. We first note that the estimates in Lemma 3.10, needed in the proof of Lemma 3.11, are worse for $d=2$ than for $d \geq 3$. The trouble comes from the small momentum regime. The function $\Theta(v)$ has a $\log |v|$ singularity for small $v$ in the real part of $\Theta$; the imaginary part is bounded. This means that the constant $C$ in Lemma 3.11 (which partly comes from (3.71)) blows up logarithmically if the zero momenta set has a positive probability with respect to the initial wavefunction. On the other hand, the Boltzmann equation (1.4) involves only $\operatorname{Im} \Theta$, which remains bounded. Hence the solution to (1.4) depends weakly continuously on its initial data, to be made precise later on. We now use this regularity property of the Boltzmann equation (1.4) and the unitarity of the Schrödinger evolution to cutoff the small momenta regime of the initial wave function.

Let $\theta>0$ be the cutoff threshold and let $\chi^{\theta}$ denote the smooth radial cutoff function supported outside the ball of radius $\theta$ centered at the origin with $\chi^{\theta}(r) \equiv 1$ for $|r| \geq 2 \theta$. We decompose the initial wave function into

$$
\psi_{0}^{\varepsilon}=\psi_{0}^{\varepsilon, \theta}+\left(\psi_{0}^{\varepsilon}-\psi_{0}^{\varepsilon, \theta}\right)
$$

where

$$
\widehat{\psi}_{0}^{\varepsilon, \theta}(r):=\widehat{\psi}_{0}^{\varepsilon}(r) \chi^{\theta}(r)
$$

We can compute the Wigner distribution of $\psi_{0}^{\varepsilon, \theta}$ easily. The proof is rather simple and will be postponed.

Lemma 6.1 . For any $\theta>0$ let $F_{0}^{\theta}(X, V)=|h(X)|^{2} \delta(V-\nabla S(X))\left[\chi^{\theta}(V)\right]^{2}$. Then the following weak limit exists and equals to $F_{0}^{\theta}(X, V)$ :

$$
\lim _{\varepsilon \rightarrow 0} W_{\psi_{0}^{\varepsilon, \theta}}^{\varepsilon}(X, V)=F_{0}^{\theta}(X, V)
$$

Let $\psi_{t}^{\varepsilon, \theta}=e^{-i t H} \psi_{0}^{\varepsilon, \theta}$ be the solution to the Schrödinger equation (1.1) with initial condition $\psi_{0}^{\varepsilon, \theta}$. Let $F_{T}^{\theta}$ be the solution to the Boltzmann equation (1.4) with the initial condition $F_{0}^{\theta}$ 
and the collision kernel given in the main theorem (1.1). The following key Lemma is a cutoff version of Theorem 1.1.

Lemma 6.2 . Let $d=2$. With the notations above, for any $\theta>0$ and $T>0$

$$
\lim _{\varepsilon \rightarrow 0} \mathbf{E} W_{\psi_{T / \varepsilon, \omega}^{\varepsilon, \theta}}^{\varepsilon}(X, V)=F_{T}^{\theta}(X, V)
$$

weakly in $\mathcal{S}\left(\mathbf{R}^{2 d}\right)$.

The proof of this Lemma will be given later on. We now take the $\theta \rightarrow 0$ limit in (6.2) to prove Theorem 1.1 for $d=2$. Recall the continuity property (2.10) of the Wigner transform with respect to the $L^{2}$ norm of the wave function. We use (2.10) with respect to the decomposition

$$
\psi_{t}^{\varepsilon}=\psi_{t}^{\varepsilon, \theta}+e^{-i t H}\left(\psi_{0}^{\varepsilon}-\psi_{0}^{\varepsilon, \theta}\right) .
$$

By the unitarity of the Schrödinger evolution we have $\left\|\psi_{t}^{\varepsilon}\right\|_{2}=1$ and

$$
\left\|e^{-i t H}\left(\psi_{0}^{\varepsilon}-\psi_{0}^{\varepsilon, \theta}\right)\right\|_{2}=\left\|\psi_{0}^{\varepsilon}-\psi_{0}^{\varepsilon, \theta}\right\|_{2} .
$$

Clearly,

$$
\limsup _{\theta \rightarrow 0}\left\|\psi_{\varepsilon \leq 1}^{\varepsilon}-\psi_{0}^{\varepsilon, \theta}\right\|_{2}=0 .
$$

Thus from (6.3), we have in particular $\left\|\psi_{t}^{\varepsilon, \theta}\right\|$ is uniformly bounded. For any $\varepsilon$ and $\theta$ we have (cf. $(2.10))$

$$
\left|\mathbf{E}\left\langle J, W_{\psi_{T / \varepsilon}^{\varepsilon}}^{\varepsilon}\right\rangle-\mathbf{E}\left\langle J, W_{\psi_{T / \varepsilon}^{\varepsilon, \theta}}^{\varepsilon}\right\rangle\right| \leq C(J) \sqrt{\mathbf{E}\left\|\psi_{t}^{\varepsilon, \theta}\right\|_{2}^{2} \mathbf{E}\left\|e^{-i t H}\left(\psi_{0}^{\varepsilon}-\psi_{0}^{\varepsilon, \theta}\right)\right\|_{2}^{2}}
$$

with a $J$-dependent constant $C(J)$, hence

$$
\lim _{\theta \rightarrow 0} \sup _{\varepsilon \leq 1}\left|\mathbf{E}\left\langle J, W_{\psi_{T / \varepsilon}^{\varepsilon}}^{\varepsilon}\right\rangle-\mathbf{E}\left\langle J, W_{\psi_{T / \varepsilon}^{\varepsilon, \theta}}^{\varepsilon}\right\rangle\right|=0 .
$$

From (6.2) and (6.4) we have that

$$
\lim _{\varepsilon \rightarrow 0} \mathbf{E} W_{\psi_{T / \varepsilon}^{\varepsilon}}^{\varepsilon}(X, V)=\lim _{\theta \rightarrow 0} F_{T}^{\theta}(X, V)
$$

weakly.

Notice that the Boltzmann evolution (1.4) is weakly continuous in the following sense: Suppose that we have a sequence of initial data $F_{0}^{\theta}(X, V)$ such that

$$
\lim _{\theta \rightarrow 0} F_{0}^{\theta}(X, V)=F_{0}(X, V)
$$


Let $F_{T}^{\theta}(X, V)$ denote the solution to the Boltzmann equation with initial data $F_{0}^{\theta}(X, V)$. Then

$$
\lim _{\theta \rightarrow 0} F_{T}^{\theta}(X, V)=F_{T}(X, V)
$$

weakly. This can be checked easily since (1.4) is a linear equation and the collision kernel is bounded in $d=2$. In our case,

$$
F_{0}^{\theta}(X, V)=|h(X)|^{2} \delta(V-\nabla S(X))\left[\chi^{\theta}(V)\right]^{2} \rightarrow|h(X)|^{2} \delta(V-\nabla S(X)):=F_{0}(X, V)
$$

weakly as $\theta \rightarrow 0$ provided that the set $\{(X, V): V=0\}$ has zero measure with respect to the probability measure $F_{0}(X, V) d X d V$. This concludes Theorem 1.1 for $d=2$.

It remains to prove Lemmas 6.1 and 6.2. The proof of Lemma 6.1 is just a simple exercise in real analysis.

Proof of Lemma 6.1. For any smooth testfunction $J$, from the definition of the Wigner distribution (1.6) we have

$$
\begin{aligned}
& \int d X d V \overline{J(X, V)} W_{\psi_{0}^{\varepsilon, \theta}}^{\varepsilon}(X, V) \\
& =\varepsilon^{-d} \int d \zeta d v \widehat{\widehat{J}(\zeta, v)} \chi^{\theta}\left(v-\frac{\zeta \varepsilon}{2}\right) \chi^{\theta}\left(v+\frac{\zeta \varepsilon}{2}\right) \int e^{i\left[\frac{(X-Y) \cdot v-(S(X)-S(Y))}{\varepsilon}-\zeta \cdot \frac{X+Y}{2}\right]} \overline{h(X)} h(Y) d X d Y
\end{aligned}
$$

By Taylor expansion there exist bounded smooth functions $F^{\theta}$ and $G^{\theta}$ such that

$$
\chi^{\theta}\left(v-\frac{\zeta \varepsilon}{2}\right) \chi^{\theta}\left(v+\frac{\zeta \varepsilon}{2}\right)=\left[\chi^{\theta}(v)\right]^{2}-\varepsilon^{2} \zeta^{2} F^{\theta}(v)+\varepsilon^{3} \zeta^{3} G^{\theta}(v, \zeta \varepsilon)
$$

Since $d=2$, we need to compute

$$
\begin{aligned}
\lim _{\varepsilon \rightarrow 0} & \int d \zeta d v \overline{\widehat{J}(\zeta, v)}\left[\varepsilon^{-2}\left[\chi^{\theta}(v)\right]^{2}+\zeta^{2} F^{\theta}(v)+\varepsilon \zeta^{3} G^{\theta}(v, \zeta \varepsilon)\right] \\
& \times \int e^{i\left[\frac{(X-Y) \cdot v-(S(X)-S(Y))}{\varepsilon}-\zeta \cdot \frac{X+Y}{2}\right]} \overline{h(X)} h(Y) d X d Y .
\end{aligned}
$$

The term with $G^{\theta}$ has an extra $\varepsilon$, so for this term one can estimate all integrands by absolute values and it gives a negligible contribution. For the other two terms, we need the following fact: for any Schwarz functions $H, I$

$$
\begin{gathered}
\lim _{\varepsilon \rightarrow 0} \varepsilon^{-2} \int d \zeta d v \overline{\widehat{I}(\zeta, v)} H(v) \int e^{i\left[\frac{(X-Y) \cdot v-(S(X)-S(Y))}{\varepsilon}-\zeta \cdot \frac{X+Y}{2}\right]} \overline{h(X)} h(Y) d X d Y \\
=\int d X|h(X)|^{2} \overline{I(X, \nabla S(X))} H(\nabla S(X))
\end{gathered}
$$


Applying this statement to $H=F^{\theta}$ and $I=-\Delta_{X} J$, we have

$$
\lim _{\varepsilon \rightarrow 0} \int d \zeta d v \widehat{\widehat{J}(\zeta, v)} \zeta^{2} F^{\theta}(v) \int e^{i\left[\frac{(X-Y) \cdot v-(S(X)-S(Y))}{\varepsilon}-\zeta \cdot \frac{X+Y}{2}\right]} \overline{h(X)} h(Y) d X d Y=0
$$

since the factor $\varepsilon^{-2}$ is missing in this expression. Applying this statement to $H=\left[\chi^{\theta}\right]^{2}$ and $I=J$, we have

$$
\begin{aligned}
& \lim _{\varepsilon \rightarrow 0} \varepsilon^{-2} \int d \zeta d v \overline{\widehat{J}(\zeta, v)}\left[\chi^{\theta}(v)\right]^{2} \int e^{i\left[\frac{(X-Y) \cdot v-(S(X)-S(Y))}{\varepsilon}-\zeta \cdot \frac{X+Y}{2}\right]} \overline{h(X)} h(Y) d X d Y \\
& =\int d X|h(X)|^{2} \overline{J(X, \nabla S(X))}\left[\chi^{\theta}(\nabla S(X))\right]^{2} .
\end{aligned}
$$

This proves Lemma 6.1.

It remains to prove (6.6). We first perform the $d \zeta$ integral and then change the variables $A=(X+Y) / 2$ and $B=X-Y$. Thus the LHS of (6.6) is equal to

$$
\lim _{\varepsilon \rightarrow 0} \varepsilon^{-2} \int d A d B d v \overline{J(A, v)} H(v) e^{i \varepsilon^{-1}\left[B \cdot v-S\left(A+\frac{B}{2}\right)+S\left(A-\frac{B}{2}\right)\right]} \overline{h\left(A+\frac{B}{2}\right)} h\left(A-\frac{B}{2}\right)
$$

¿From the Taylor expansion, the exponent can be written as

$$
i \varepsilon^{-1}\left[B \cdot v-S\left(A+\frac{B}{2}\right)+S\left(A-\frac{B}{2}\right)\right]=i \varepsilon^{-1}\left[B \cdot(v-\nabla S(A))+B^{2} K(A, B)\right],
$$

where $K(A, B)$ is some bounded smooth function. Changing the variable $B=\varepsilon^{-1} \beta$, we have that $(6.7)$ is equal to

$$
\begin{aligned}
& \lim _{\varepsilon \rightarrow 0} \int d A d \beta d v \overline{J(A, v)} H(v) e^{i \beta \cdot(v-\nabla S(A))+i \varepsilon \beta^{2} K(A, \varepsilon \beta)} \overline{h\left(A+\frac{\varepsilon \beta}{2}\right)} h\left(A-\frac{\varepsilon \beta}{2}\right) \\
& =\int d A d \beta d v \overline{J(A, v)} H(v) e^{i \beta \cdot(v-\nabla S(A))}|h(A)|^{2}
\end{aligned}
$$

The last term is the right hand side of (6.6). This completes the proof of Lemma 6.1.

We now prove Lemma 6.2. As remarked in the begining of this section, we shall only prove Lemmas 3.5-3.12 for $d=2$ with the initial wave function supported outside a ball of radius $\theta$ in the Fourier space. Lemma 3.6 can be proved in exactly the same way. The key steps to prove Lemma 3.7 are Lemmas 3.11 and 3.12; the rest of the arguments to prove Lemma 3.7 hold for $d=2$ as well. Thus we only have to prove Lemmas 3.5, 3.11 and 3.12. We shall omit all superscripts $\theta$ from the notations for the rest of this section. All constants will depend on $\theta$. Certainly, the support of $\widehat{\psi}_{0}$ is now outside a ball of radius $\theta$ in the Fourier space. 
Proof of Lemma 3.5 for $d=2$ with cutoff initial function. Following the original proof, we notice that the estimate (3.38) needs to be modified in $d=2$. It is easy to see that (3.38) remains true with an extra

$$
\frac{1}{|\alpha|+t^{-1}}+\frac{1}{|\beta|+t^{-1}}
$$

factor on the right hand side.Thus the same factor appears in (3.39). Since the initial wave function is supported outside a ball of radius $\theta$, we can restrict the $w_{0}$ integration in (3.39) to $\left|w_{0}\right| \geq \theta$. This cutoff ensures that the two singularities, $\alpha \sim 0$ and $\alpha \sim w_{0}^{2} / 2$ are separated. Thus the $d \alpha$ integration gives only an extra $\log t$ factor. The same argument applies to the $d \beta$ integration. This proves Lemma 3.5 for $d=2$ with cutoff initial wave function.

We now prove Lemma 3.11 and 3.12 for $d=2$.

Proof of Lemma 3.11 for $d=2$ with cutoff initial function $\psi_{0}^{\varepsilon, \theta}$. We first note that the uniform bound (3.71) is replaced in $d=2$ by

$$
\left|\Theta_{\alpha, \eta}(r)\right| \leq C\left(1+[\log \alpha]_{-}\right) .
$$

Hence $(3.72)$ is modified by the factor $\left(1+[\log \alpha]_{-}\right)^{\|\mathbf{k}\|+m}\left(1+[\log \beta]_{-}\right)^{\left\|\mathbf{k}^{\prime}\right\|+m}$ on the right hand side, i.e.,

$$
\left|G_{\eta}\left(\alpha, \beta, \mathbf{r}, \mathbf{r}, \mathbf{k}, \mathbf{k}^{\prime}\right)\right|\left|\widehat{\psi}_{0}\left(r_{m}\right)\right|^{2} d \mathbf{r}_{m} \leq C^{\|\mathbf{k}\|+\left\|\mathbf{k}^{\prime}\right\|+2 m}\left(1+[\log \alpha]_{-}\right)^{\|\mathbf{k}\|+m}\left(1+[\log \beta]_{-}\right)^{\left\|\mathbf{k}^{\prime}\right\|+m} d \mu\left(\mathbf{r}_{m}\right)
$$

Thus from (3.63)

$$
\begin{aligned}
\left|C_{\pi}\right| \leq & (C \lambda)^{2 \bar{n}} e^{t \eta} \int_{\left|r_{m}\right| \geq \theta} d \mu\left(\mathbf{r}_{m}\right) \int_{-\infty}^{\infty} d \alpha d \beta \\
& \prod_{j=0}^{m}\left|\frac{1}{\alpha-r_{j}^{2} / 2-i \eta}\right|^{k_{j}+1}\left|\frac{1}{\beta-r_{j}^{2} / 2+i \eta}\right|^{k_{j}^{\prime}+1}\left(1+[\log \alpha]_{-}\right)^{\|\mathbf{k}\|+m}\left(1+[\log \beta]_{-}\right)^{\left\|\mathbf{k}^{\prime}\right\|+m} .
\end{aligned}
$$

The goal is to show that $(6.9)$ is bounded by $\left(C \lambda^{2} t\right)^{\bar{n}}$. The constant may depend on $\theta$. Let

$$
\mathcal{B}:=\left\{(\alpha, \beta):|\alpha| \geq \frac{\theta^{2}}{16},|\beta| \geq \frac{\theta^{2}}{16}\right\}
$$

and denote by $\mathcal{B}^{c}$ the complement of $\mathcal{B}$. Define

$$
\begin{aligned}
\mathcal{F}_{\mathcal{B}}:= & (C \lambda)^{2 \bar{n}} e^{t \eta} \int_{\left|r_{m}\right| \geq \theta} d \mu\left(\mathbf{r}_{m}\right) \int_{(\alpha, \beta) \in \mathcal{B}} d \alpha d \beta \\
& \prod_{j=0}^{m}\left|\frac{1}{\alpha-r_{j}^{2} / 2-i \eta}\right|^{k_{j}+1}\left|\frac{1}{\beta-r_{j}^{2} / 2+i \eta}\right|^{k_{j}^{\prime}+1}\left(1+[\log \alpha]_{-}\right)^{\|\mathbf{k}\|+m}\left(1+[\log \beta]_{-}\right)^{\left\|\mathbf{k}^{\prime}\right\|+m}
\end{aligned}
$$


and similarly define $\mathcal{F}_{\mathcal{B}^{c}}$. We have the following extension of Lemma 3.11. This proves Lemma 3.11 for $d=2$.

Lemma 6.3 . For any initial function and any $\pi \in \Pi_{n, n^{\prime}}$ with $n+n^{\prime} \leq 2 n_{0}(\varepsilon)$ we have

$$
\begin{gathered}
\left|\mathcal{F}_{\mathcal{B}}\right| \leq\left(C \lambda^{2} t\right)^{\bar{n}} . \\
\left|\mathcal{F}_{\mathcal{B}^{c}}\right| \leq t^{-1 / 2}\left(C \lambda^{2} t\right)^{\bar{n}} .
\end{gathered}
$$

Proof of Lemma 6.3. We follow the proof of Lemma 3.11. Since $(\alpha, \beta) \in \mathcal{B}$, the logarithmic factors $\left(1+[\log \alpha]_{-}\right)^{\|\mathbf{k}\|+m}\left(1+[\log \beta]_{-}\right)^{\left\|\mathbf{k}^{\prime}\right\|+m}$ is bounded by $C^{2 \bar{n}}$. Thus we can directly follow the proof of Lemma 3.11 to obtain a bound $\left(C \lambda^{2} t\right)^{\bar{n}}$ for $\mathcal{F}_{\mathcal{B}}$. This proves (6.11).

We now prove (6.12). We first define the quantity

$$
\widetilde{\Sigma}(t, \mathbf{p}, \mathbf{k}):=\int_{-\infty}^{\infty} d \alpha\left(1+[\log \alpha]_{-}\right)^{\|\mathbf{k}\|+m} \prod_{j=0}^{m}\left|\frac{1}{\alpha-p_{j}^{2} / 2-i t^{-1}}\right|^{k_{j}+1} .
$$

which is a modification of $\Sigma$ defined in (3.26). The estimate (3.28) has the version for $\tilde{\Sigma}$ :

$$
\int|\widetilde{\Sigma}(t, \mathbf{p}, \mathbf{k})|^{2} d \mu\left(\mathbf{p}_{m}\right) \leq t^{\frac{1}{4}}(C t)^{m+2\|\mathbf{k}\|} .
$$

To prove it, we replace $R\left(\alpha, p_{m-1}, p_{m}\right)$ in (3.17) by

$$
\widetilde{R}\left(\alpha, p_{m-1}, p_{m}\right):=\left(1+[\log \alpha]_{-}\right)^{m+\|\mathbf{k}\|} R\left(\alpha, p_{m-1}, p_{m}\right)
$$

and replace $Z\left(p_{m-1}, p_{m}\right)$ in (3.18) by

$$
\widetilde{Z}\left(p_{m-1}, p_{m}\right):=\int_{-\infty}^{\infty} \frac{\left(1+[\log \alpha]_{-}\right)^{m+\|\mathbf{k}\|}}{\left|\alpha-p_{m-1}^{2} / 2+i \eta\right|\left|\alpha-p_{m}^{2} / 2+i \eta\right|} d \alpha .
$$

Recall that $m+\|\mathbf{k}\| \leq n \leq 2 \bar{n} \leq 2 n_{0}(\varepsilon)$ with $n_{0}(\varepsilon)$ defined in (2.2) and satisfying (2.3). Simple calculation shows that the estimate (3.19) is valid for $\widetilde{Z}$ with an extra $t^{1 / 4}$ factor on the right hand side. Here we have to use (2.3). This proves (6.13).

Recall $(\alpha, \beta) \in \mathcal{B}^{c}$ for $\mathcal{F}_{\mathcal{B}^{c}}$, hence $|\alpha| \leq \theta^{2} / 8$ or $|\beta| \leq \theta^{2} / 8$. Suppose we have $|\alpha| \leq \theta^{2} / 8$. Since we only integrate $\left|r_{m}\right| \geq \theta$ in (6.9) the denominator

$$
\left|\alpha-r_{m}^{2}+i \eta\right| \geq \theta^{2} / 8
$$


Notice that this reduces $k_{m}$ by 1 . We now follow the proof of Lemma 3.11 but use (6.13) and the remark above. Since $k_{m}$ is reduced by 1 , we we obtain an extra $t^{-1}$. Together with the factor $t^{1 / 4}$ in (6.13), we have an overall factor $t^{-3 / 4} \leq t^{-1 / 2}$. This proves (6.12).

Proof of Lemma 3.12 for $d=2$ with cutoff initial function. We only remark the modifications compared with the $d \geq 3$ case. Our goal is to estimate (3.76) for $d=2$. Notice that $\left|G_{\eta}\left(\alpha, \beta, \mathbf{r}, \mathbf{r}, \mathbf{k}, \mathbf{k}^{\prime}\right)\right|\left|\widehat{\psi}_{0}\left(r_{m}\right)\right|^{2} d \mathbf{r}_{m}$ satisfies (6.8). Similar inequality holds if we replace $G_{\eta}\left(\alpha, \beta, \mathbf{r}, \mathbf{r}, \mathbf{k}, \mathbf{k}^{\prime}\right)$ by $|M(\mathbf{r}, m)|^{2} \overline{\Theta\left(r_{0}\right)}\|\mathbf{k}\| \mid \Theta\left(r_{0}\right)^{\left\|\mathbf{k}^{\prime}\right\|}$. Thus from (6.12) in Lemma 6.3 the contribution of the set $(\alpha, \beta) \in \mathcal{B}^{c}$ to $(3.76)$ is negligible. We only have to consider the case $(\alpha, \beta) \in \mathcal{B}$. Using Lemma 3.10, we have to modify (3.78) to

$$
\left|\Theta_{\alpha, \eta}^{*}(|r|)-\Theta_{\alpha, \eta}^{*}\left(\left|r^{\prime}\right|\right)\right| \leq C|| r|-| r^{\prime}||\left(1+[\log \alpha]_{-}\right)
$$

Thus (3.79) becomes

$$
\begin{aligned}
\Theta_{\alpha, \eta}\left(r_{j}\right)-\Theta\left(r_{0}\right) \leq & C|\alpha|^{-1 / 2}\left(1+[\log \alpha]_{-}\right)\left(\left|\alpha-\frac{r_{j}^{2}}{2}\right|+\left|\alpha-\frac{r_{0}^{2}}{2}\right|\right) \\
& +C\left(\eta^{-1 / 2}+\frac{1}{|\alpha|+\eta}+\frac{1}{\left|r_{0}\right|}\right)\left(\left|\alpha-r_{0}^{2} / 2\right|+\eta\right) .
\end{aligned}
$$

Hence we can bound (3.76) on the set $(\alpha, \beta) \in \mathcal{B}$ by

$$
\begin{aligned}
& C^{m} \int d \mu\left(\mathbf{r}_{m}\right) \int_{(\alpha, \beta) \in \mathcal{B}} d \alpha d \beta \prod_{j=0}^{m}\left|\frac{1}{\alpha-r_{j}^{2} / 2-i \eta}\right|^{k_{j}+1}\left|\frac{1}{\beta-r_{j}^{2} / 2+i \eta}\right|^{k_{j}^{\prime}+1} \\
& \times\left(1+[\log \alpha]_{-}\right)^{m+\|\mathbf{k}\|}\left(1+[\log \beta]_{-}\right)^{m+\left\|\mathbf{k}^{\prime}\right\|} \\
& \times\left[\eta^{-1 / 2}+\frac{1}{|\alpha|^{1 / 2}}+\frac{1}{|\alpha|+\eta}+\frac{1}{|\beta|^{1 / 2}}+\frac{1}{|\beta|+\eta}+\frac{1}{\left|r_{0}\right|}\right] \\
& \times\left[O\left(\alpha-r_{0}^{2} / 2\right)+O\left(\beta-r_{0}^{2} / 2\right)+\sum_{j}\left\{O\left(\alpha-r_{j}^{2} / 2\right)+O\left(\beta-r_{j}^{2} / 2\right)+O(\eta)\right\}\right]
\end{aligned}
$$

As in the proof for $d \geq 3$, we gain an extra factor $t^{-1}$ from the terms in the second square bracket in (6.17). Again, we can estimate the logarithmic factors $\left(1+[\log \alpha]_{-}\right)^{\|\mathbf{k}\|+m}(1+$ $\left.[\log \beta]_{-}\right)^{\left\|\mathbf{k}^{\prime}\right\|+m}$ in $\mathcal{B}$ by $C^{\bar{n}}$. Thus we can estimate (6.17) following the proof for $d \geq 3$. This concludes the proof of Lemma 3.12 for $d=2$. 


\section{Appendix.}

Proof of Lemma 2.3. Part (i). Using the w variables, all Type (I), (I'), (II) deltafunctions can be written in a unified way as $\delta\left(w_{i}-w_{i+1}+w_{j}-w_{j+1}\right)$ with $0 \leq i<j \leq 2 \bar{n}$, and the Type $\operatorname{III}(\xi)$ delta function is $\delta\left(w_{n}-w_{n+1}+\xi\right)$. Notice that for this proof it suffices to consider the case $\xi=0$, the extension to nonzero $\xi$ is obvious.

From these deltafunctions one can form a $(\bar{n}+1) \times(2 \bar{n}+2)$ matrix $\mathcal{M}$ in a natural way; the columns correspond to variables $w_{0}, w_{1}, \ldots w_{2 \bar{n}+1}$, each row corresponds to a deltafunction and it contains $0,+1,-1$ according to the coefficients in the linear combination of $w$ 's given in the deltafunction. The product of all the deltafunctions gives the kernel of $\mathcal{M}$, as a subspace of $\mathbf{R}^{2 \bar{n}+2}$ (coordinatized by the variables $w_{0}, w_{1}, \ldots w_{2 \bar{n}+1}$ ).

The proof of Lemma 2.3 is a simple Gaussian elimination performed on the matrix $\mathcal{M}$. We shall bring the matrix into a form $\left[\mathcal{M}_{0} \mid-I\right]$, where $I$ is the $(\bar{n}+1) \times(\bar{n}+1)$ identity matrix and $\mathcal{M}_{0}$ is the matrix in (2.19). The columns in the $-I$ part of that matrix correspond to the variables indexed by the set $B$. Using the $-I$ matrix, this means that the rows of $\mathcal{M}_{0}$ can also be associated with the elements of $B$. From this elimination, in particular, it follows that the rank of $\mathcal{M}$ is $\bar{n}+1$.

Since we have to show some particular properties of $\mathcal{M}_{0}$, a simple existence argument is not sufficient. It is simpler to give an explicit construction.

Each delta function $\delta\left(w_{i}-w_{i+1}+w_{j}-w_{j+1}\right)$, in its original (non simplified) form, contains four momentum variables, indexed by $i<i+1 \leq j<j+1$. Two of these appear with plus sign, two with minus sign and the two middle ones can cancel each other. In this case, the corresponding row of $\mathcal{M}$ contain only two nonzero entries. The variable with the biggest index $j+1$ always comes with a minus sign, and this index is called leading. Furthermore, the leading index of the Type III delta function $\delta\left(w_{n}-w_{n+1}\right)$ is $n+1$ by definition. Obviously, the leading indices are different for different delta functions. Define $B$ to be the set of all leading indices, $|B|=\bar{n}+1$, and let $A$ be the complementary set of indices. It is clear from the construction that $0 \in A$ and $2 \bar{n}+1 \in B$.

We show that by permuting rows and by adding certain rows to some others in $\mathcal{M}$, we can achieve that

(i) all the columns, corresponding to $B$-elements, have only a single -1 nonzero entry;

(ii) all entries of the matrix are $0,-1$ or 1 ; 
(iii) the leading -1 is last nonzero element in each row ;

(iv) the last row is $(1,0,0, \ldots, 0,-1)$.

It is obvious that with these row operations, the sum of the elements in each row remains 0 . Hence, after rearranging the rows and columns, this will yield the form $\left[\mathcal{M}_{0} \mid-I\right]$, with a matrix $\mathcal{M}_{0}$ described in Lemma 2.3.

By looking at the description of the deltafunctions we observe that in the matrix $\mathcal{M}$ the first column contains only a +1 nonzero entry, the last column contains a single -1 and all other columns are either completely empty or they contain exactly one +1 and one -1 . In particular, the sum of all rows is $(1,0,0, \ldots, 0,-1)$. The $B$-columns, i.e. the $b_{1}$-th, $b_{2}$-th etc. ones, are never empty. For each $b \in B, b \neq 2 \bar{n}+1$, let $R_{b}^{+}$(and $R_{b}^{-}$) to be the row which contains $\mathrm{a}+1\left(-1\right.$, respectively) in the $b^{\text {th }}$ place.

We permute the rows of $\mathcal{M}$ such that $b \mapsto R_{b}^{-}$be an increasing function. We put the row containing the leading -1 in the last column into the last row of the permuted matrix. Since the leading -1 's are the last nonzero elements in each row, we see that $R_{b}^{-}<R_{b}^{+}$for each $b \in B, b \neq 2 \bar{n}+1$. For each such $b$, we add the row $R_{b}^{-}$to the row $R_{b}^{+}$and we do it in the increasing order of the $b$ 's. More precisely, if $b_{1}<b_{2}<\ldots<b_{\bar{n}}$ are the elements of $B$ which differ from $2 \bar{n}+1$, then first we add the row $R_{b_{1}}^{-}$to $R_{b_{1}}^{+}$, then the row $R_{b_{2}}^{-}$to $R_{b_{2}}^{+}$, etc. We name these steps according to their column index as $b_{1}$-step, $b_{2}$-step, etc.

In this way, each leading -1 , except the one in the last row, is added exactly once to cancel the +1 in its own column below it. Hence after these operations, the $B$-columns will contain a single -1 . The column which originally contained a single -1 is not affected. Notice that no leading -1 is cancelled. This is a consequence of the following two facts:

(i) we always add a row to some other row below;

(ii) during the elimination, the elements above a leading -1 in its column are always zero. The conclusion is that after these operations, the resulting matrix has a single -1 in each $B$-column. Furthermore, these -1 's are the last nonzero elements in their rows and the first nonzero elements in their column.

The last row of the resulting matrix is $(1,0,0, \ldots, 0,-1)$. For, along the elimination each row but the last one has been added exactly once to some other row below. Hence, at the end, the last row will be the sum of all the rows of the original matrix.

Finally, we have to show that the only possible entries are $0,-1$ and +1 . Suppose this is not the case. Since the entries originally were $0,-1,+1$, we can consider the first moment 
during the elimination procedure, when a \pm 2 was created. For definiteveness, assume that it was in the $b$-step (when row $R_{b}^{-}$was added to row $R_{b}^{+}$), that an entry +2 appeared at the first time, and suppose that it was in the $m^{\text {th }}$ column (the proof for the case -2 is identical). In other words, right before the $b$-step both rows $R_{b}^{-}$and $R_{b}^{+}$contained +1 at the $m^{\text {th }}$ place. Certainly $m<b$ as $R_{b}^{-}$has zero elements in the columns with higher index than $b$.

During the elimination, we call a row untouched if it has not been added yet to any other row (but some other row could have been added to it). In the original matrix $\mathcal{M}$ there was at most one +1 in the $m^{\text {th }}$ column and suppose it was in the row $R$. Due to the algorithm, both +1 's at the $m^{\text {th }}$ place at the $b$-step must originate from this single +1 .

Clearly $R \leq R_{b}^{-}$, since every row is added at most once to some other row, always below, hence the +1 originally in the row $R$ could not appear in a row above it. For the same reason, $R \neq R_{b}^{-}$, since it is exactly the $b$-step when we add the $R_{b}^{-}$row. Before this step, the $R_{b}^{-}$ row was untouched and the +1 in the this row has not been moved out. So the +1 in the $R_{b}^{+}$row could not have been created if $R=R_{b}^{-}$. Hence, right before the $b$-step there are two untouched rows (namely $R_{b}^{+}$and $R_{b}^{-}$), both contain a +1 at the $m^{t h}$ place which were not there in the original matrix $\mathcal{M}$.

We show that this is impossible. Notice that there could several +1 's appear in the same column along the elimination. But at every moment, there is at most one of them which sits in an untouched row. This is seen by induction on the step number. Whenever a new +1 is created in a column, it must come from another +1 in the same column, and this latter +1 sits in a row which has just been touched. Hence the number of +1 's in untouched rows never increases. Originally there was at most one +1 in the $m^{\text {th }}$ column, so there could not be two of them before the $b$-step.

The proof of part (ii) is very similar to the case $r=\bar{n}+1$ and the details are left to the reader. This completes the proof of Lemma 2.3.

Proof of Lemma 2.6. First we show that the identifications described in the theorem can be forced. Fix two edges $e\left(w_{i}\right)$ and $e\left(w_{j}\right)$ and suppose that after removing them, the graph $G=G_{\pi}$ splits into two components: $G \backslash\left\{e\left(w_{i}\right), e\left(w_{j}\right)\right\}=G_{1} \cup G_{2}, G_{1} \cap G_{2}=\emptyset$ (as graphs). We used that $G$ contained the circle graph $C$ with $V(G)=V(C)$, hence $G$ is two-connected, 
so the number of components after removal of two edges is at most two. Choose the indices such that the right square be in $V\left(G_{2}\right)$.

Notice that $G_{i}(i=1,2)$ cannot be just a single bullet (it can be a square), since each bullet has three edges adjacent to them. This corresponds to the fact that $w_{i}=w_{i+1}$ type momentum identifications are not possible (apart from the momenta at the squares). $G_{i}$ certainly can be a square, but this leads to the trivial deltafunction $\delta\left(w_{2 \bar{n}+1}-w_{0}\right)$ or $\delta\left(w_{n}-w_{n+1}\right)$ which are forced as we have seen. So from now on we can exclude this case.

Let $C \backslash\left\{e\left(w_{i}\right), e\left(w_{j}\right)\right\}=A_{1} \cup A_{2}$ (as graphs), where $V\left(A_{i}\right)=V\left(G_{i}\right)$, i.e. the arc (directed path) $A_{i}$ is a subgraph of $G_{i}$. Obviously $A_{1} \cap A_{2}=\emptyset$ (as graphs), and since $G_{1} \cap G_{2}=\emptyset$, it is clear that all the pairing edges in the original $G$ must be either entirely within $G_{1}$ or in $G_{2}$. On Fig. $5 i=2, j=8, A_{1}$ is the directed arc with edges $e\left(w_{3}\right), e\left(w_{4}\right), \ldots, e\left(w_{7}\right)$ and $A_{2}$ is $e\left(w_{9}\right), e\left(w_{10}\right), \ldots e\left(w_{2 \bar{n}+1}\right), e\left(w_{0}\right), e\left(w_{1}\right)$.

Consider $G_{1}$. All pairing lines starting from any point of $V\left(G_{1}\right)$ must be joined to another bullet from $V\left(G_{1}\right)$. Let us add up the arguments of all deltafunctions (Type I, I' and II) which correspond to pairing lines within $G_{1}$, and if $G_{1}$ also contains the left square, then add the argument of $\delta\left(w_{n}-w_{n+1}\right)$ as well. Let us call this collection of deltafunctions $\Delta$. Notice that in all deltafunctions the sign is decided by the arrow: the sign is plus if the arrow is incoming (into the vertex, associated with the deltafunction) and minus otherwise.

In $\Delta$ every momentum belonging to $E\left(G_{1}\right)$ appears exactly twice: once with plus once with minus sign. For, notice that every vertex (except the left square) has a unique delta function associated to it; the one whose pairing line meets this vertex. The Type III delta function is associated to the left square. Consider now the momentum $w_{m}$ with $e\left(w_{m}\right) \in E\left(G_{1}\right)$. This connects two vertices of $V\left(G_{1}\right)$. Both delta functions corresponding to these vertices are present in $\Delta$. Hence the momentum $w_{m}$ appears exactly twice in the collection $\Delta$, and since it corresponded to an arrow, which is outgoing from one vertex and incoming into the other, the sign of these two appearances are opposite. Hence when summing up the arguments of all the deltafunctions in $\Delta$, all these momenta cancel.

After summation, only those momenta remain which appear in $\Delta$, but are not part of $E\left(G_{1}\right)$. Since $\Delta$ consisted of delta functions associated to vertices in $V\left(G_{1}\right)$, the remaining momenta are those which are associated to edges in $E(G) \backslash E\left(G_{1}\right)$ and which edges are adjacent to $V\left(G_{1}\right)$, i.e. exactly $e\left(w_{i}\right)$ and $e\left(w_{j}\right)$. One of them must be incoming into $G_{1}$, the other is outgoing, since at every vertex we have one incoming and one outgoing arrow, so 
the same is true for any subset of $V(G)$, in particular for $V\left(G_{1}\right)$. This completes the proof that the given deltas force $w_{i}=w_{j}$. On Fig. 5 , the arguments of the three delta functions, $\delta\left(w_{2}-w_{3}+w_{5}-w_{6}\right), \delta\left(w_{3}-w_{4}+w_{4}-w_{5}\right), \delta\left(w_{6}-w_{7}+w_{7}-w_{8}\right)$, add up to $w_{2}-w_{8}$.

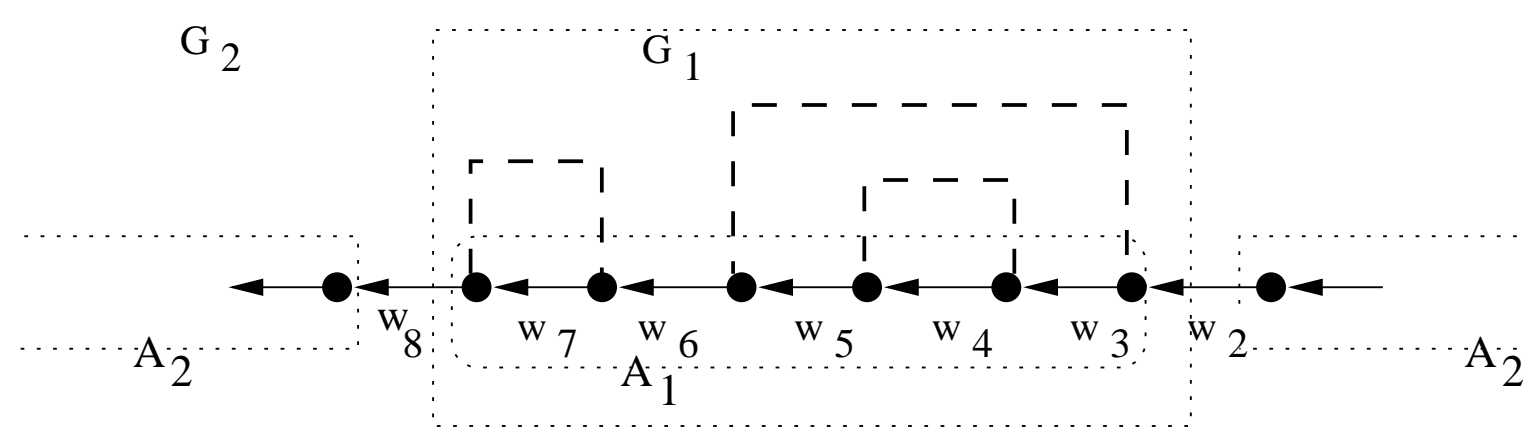

Fig. 5

For the proof of the other direction, we have to show that if some linear combination of the arguments of the delta functions Type I, I', II, forces $w_{i}=w_{j}$, then $G \backslash\left\{e\left(w_{i}\right), e\left(w_{j}\right)\right\}$ has two components (see Fig. 6).

Since $C$ is a circle, $C \backslash\left\{e\left(w_{i}\right), e\left(w_{j}\right)\right\}=A_{1} \cup A_{2}$ where $A_{1} \cap A_{2}=\emptyset$ are two arcs. Hence the two possible components of $G$, after the removal, must have the same vertex sets as the arcs: $V\left(A_{1}\right)=V\left(G_{1}\right)$ and $V\left(A_{2}\right)=V\left(G_{2}\right)$. So we have to prove that if $w_{i}=w_{j}$ is forced, then there could be no pairing line between $V\left(A_{1}\right)$ and $V\left(A_{2}\right)$ (which could save the connectedness of $\left.G \backslash\left\{e\left(w_{i}\right), e\left(w_{j}\right)\right\}\right)$.

Suppose that $G \backslash\left\{e\left(w_{i}\right), e\left(w_{j}\right)\right\}$ is connected. Then there is a pairing line $L$ which connects a vertex in $V\left(A_{2}\right)$, say $P$, with a vertex in $V\left(A_{1}\right)$, say $Q$. Of course $P$ and $Q$ are bullets (not squares; as there is no pairing line coming out of a square). Consider $C_{L}:=C \cup L$, more precisely, $C_{L}$ is a graph such that $V\left(C_{L}\right)=V(C)$ and $E\left(C_{L}\right)=E(C) \cup\{L\}$. This is a circle with an extra loop $L$. Since $L$ connects $A_{1}$ and $A_{2}$, there is a circle $\bar{C}$ in $C_{L}$ which contains the edge $e\left(w_{i}\right)$, but not $e\left(w_{j}\right)$ and $\bar{C}$ naturally contains $L . \bar{C}$ contains an $\operatorname{arc} A$ from $C$ built from momentum lines (the arc $A$ between $P$ and $Q$ ), and clearly $\bar{C}=A \cup L$. 


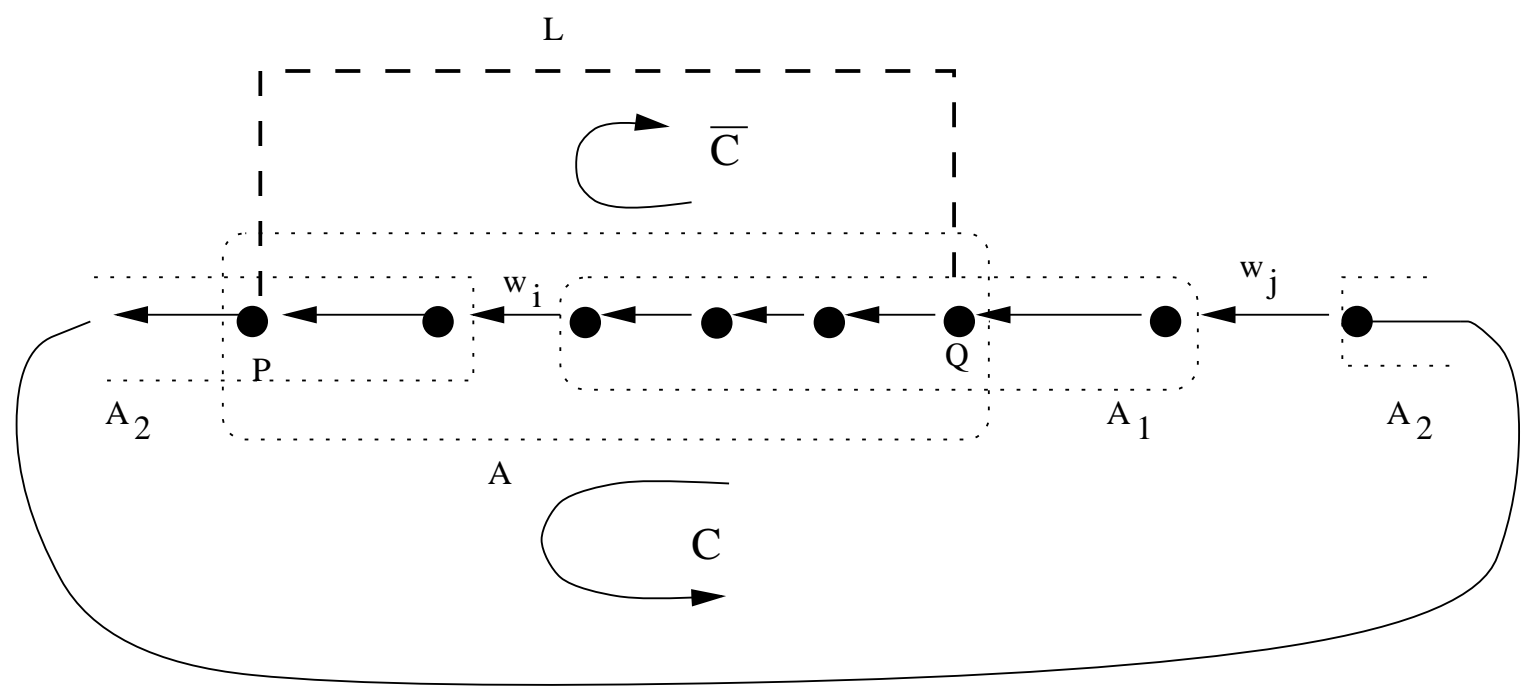

Fig. 6

Now we add a nonzero momentum $q$ to all the momenta associated with the momentum lines in $A$ and leave the momenta associated with the complement $\operatorname{arc} A^{c}:=C \backslash A$, containing $e\left(w_{j}\right)$, untouched. We claim that this shift in the momenta is compatible with all the deltafunctions Type I, I', II, III but is not compatible with $\delta\left(w_{i}-w_{j}\right)$, hence the latter cannot be forced by our deltas.

It is clear that this shift is not compatible with $\delta\left(w_{i}-w_{j}\right)$, since after the shift $w_{i} \rightarrow w_{i}+q$, while $w_{j}$ is unchanged.

To see that this momentum shift is compatible with all our deltas, first note that if a deltafunction (from Type I, I', II) is not the one associated with $P$ or $Q$, then it remains valid after the shift. The reason is that this deltafunction joins two bullets, and for each bullet there is an incoming and an outgoing arrow. Depending on whether the bullet belongs to $V(A)$ or not, these two momenta get shifted or not simultaneously. But the structure of the deltafunctions is such that the incoming and the outgoing momenta come with different signs, hence either both are shifted or none of them, the deltafunction remains valid. The Type III deltafunction clearly remains valid.

Finally we have to check the validity of the delta function connecting $P$ and $Q$. But in the argument of this delta function there are two momenta which are in $A$ and two which are in $A^{c}$, and the ones which are in $A$ come with different signs. So adding $q$ to the momenta in $A$ does not change this delta function either. The proof is completed. 
Proof of Lemma 2.7. In this proof we use the notation $w_{0}, w_{1}, \ldots, w_{2 \bar{n}+1}$. In the matrix $\left[\mathcal{M}_{0} \mid-I\right]$, the first $\bar{n}+1$ columns are associated with the elements of $A$, the remaining columns with the elements of $B$. Hence, by the identity matrix, the rows of $\mathcal{M}_{0}$ can be associated with the elements of $B$ as well.

Suppose first that $\pi$ is crossing, i.e. it contains two delta functions $\delta\left(w_{i}-w_{i+1}+w_{j}-w_{j+1}\right)$ and $\delta\left(w_{k}-w_{k+1}+w_{\ell}-w_{\ell+1}\right)$ such that $i<k<j<\ell$. By definition, $j+1 \in B$, i.e. $j+1=b_{\nu}$ with some $1 \leq \nu \leq \bar{n}+1$ (see the proof of Lemma 2.3). Suppose that in $\mathcal{M}_{0}$, the $\nu$-th row (associated with $j+1 \in B$ ) contains only one nonzero element. Then $\Delta_{0}^{\pi}(\mathbf{w})$ forces the identification $w_{j+1}=w_{u}$ for some $u \in A$. Moreover, $u<j+1$ by Lemma 2.3. Using Lemma 2.6, we obtain that the removal of the edges $w_{u}$ and $w_{j+1}$ disconnects $G_{\pi}$. But this is impossible; the two dashed lines corresponding to these two delta functions keep the graph connected. This can easily be checked by distinguishing three cases: $u \leq i, i+1 \leq u \leq k$ and $k+1 \leq u<j$. Fig 7 . shows these three possibilities for $w_{u}$.

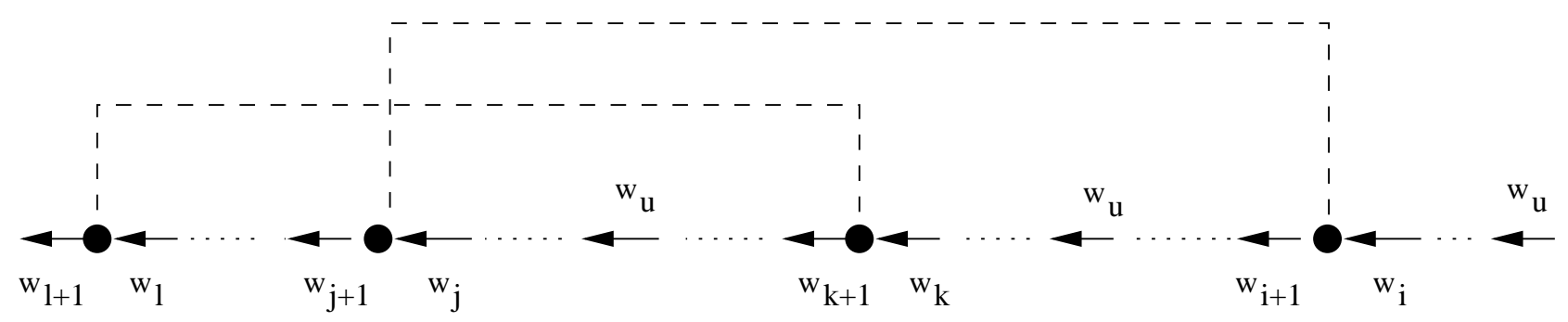

Fig. 7

For the converse proof, assume that $\pi$ is noncrossing. The set $B$ is uniquely given by $\pi$. We need the following auxiliary lemma, whose proof is postponed.

Lemma 6.4 . If $\pi$ is noncrossing, then $\Delta_{\pi}^{0}(\mathbf{w})$ is equivalent to a product of identification delta functions of the form

$$
\prod_{b \in B} \delta\left(w_{b}-w_{a(b)}\right)
$$

where $a(b) \in A$. (The sets $A, B$ are defined in Lemma 2.3.)

From the elements $a(b)$ one can form a matrix $\widetilde{\mathcal{M}}_{0}$ as follows. The $\nu$-th row of $\widetilde{\mathcal{M}}_{0}$ is associated with the element $b_{\nu} \in B$, and the $\mu$-th column with $a_{\mu} \in A$, such that the $\mu$-th entry of the $\nu$-th row is 1 for $a\left(b_{\nu}\right)=a_{\mu}$, and all other entries are zero. We claim that $\widetilde{\mathcal{M}}_{0}$ is the same as $\mathcal{M}_{0}$ obtained in Lemma 2.3. If it were not the case, then for some 
$b_{\nu} \in B$, the original $\Delta_{\pi}^{0}(\mathbf{w})$ would force both $\delta\left(w_{b_{\nu}}-w_{a_{\mu}}\right)$ and $\delta\left(w_{b_{\nu}}-\sum_{\alpha \in A^{*}}\left( \pm w_{\alpha}\right)\right)$ with some $A^{*} \subset A, A^{*} \neq\left\{a_{\mu}\right\}$. But then $\Delta_{\pi}^{0}(\mathbf{w})$ would force the nontrivial delta function $\delta\left(w_{a_{\mu}}-\sum_{\alpha \in A^{*}}\left( \pm w_{\alpha}\right)\right)$ involving momenta only from $A$. This delta function obviously cannot be forced by $\prod_{\nu=1}^{\bar{n}+1} \delta\left(w_{b_{\nu}}-w_{a\left(b_{\nu}\right)}\right)$ but this latter product is equivalent to $\Delta_{\pi}^{0}(\mathbf{w})$, which contradiction proves $\widetilde{\mathcal{M}}_{0}=\mathcal{M}_{0}$, hence the converse statement of the Lemma.

Proof of Lemma 6.4. It is enough to show that for each $b \in B$ there is an $a(b) \in A$ such that $\delta\left(w_{b}-w_{a(b)}\right)$ is forced by $\Delta_{\pi}^{0}(\mathbf{w})$. For, by comparing the ranks of the product (6.18) and $\Delta_{\pi}^{0}(\mathbf{w})$, we see that these are equivalent.

Since $b \in B$, we know that a pairing line ends at the beginning of the arrow $w_{b}$. Consider the arrow at the beginning of this pairing line (see Fig. 8). In other words, suppose that this pairing line corresponded to $\delta\left(w_{k_{1}}-w_{k_{1}+1}+w_{b-1}-w_{b}\right)$ with $k_{1}<b-1$, and consider $w_{k_{1}}$. Since $\pi$ is noncrossing, the removal of $w_{k_{1}}, w_{b}$ disconnects the graph, hence $\delta\left(w_{k_{1}}-w_{b}\right)$ is forced by $\Delta_{\pi}^{0}(\mathbf{w})$ using Lemma 2.6. If $k_{1} \in A$, then we define $a(b):=k_{1}$. If $k_{1} \in B$, then we continue the procedure; there is a pairing line ending at $w_{k_{1}}$ and starting at, say, $w_{k_{2}}$. I.e. there is a delta function $\delta\left(w_{k_{2}}-w_{k_{2}+1}+w_{k_{1}-1}-w_{k_{1}}\right), k_{2}<k_{1}-1$, and we see that $\delta\left(w_{k_{2}}-w_{k_{1}}\right)$ is forced, hence $\delta\left(w_{k_{2}}-w_{b}\right)$ is forced as well. Again, if $k_{2} \in A$, then $a(b):=k_{2}$, otherwise we continue the procedure to find $k_{3}$ etc. Since $0 \in A$, we see that at some point this procedure terminates, yielding the value $a(b) \in A$ (on Fig. $8, b, k_{1}, k_{2} \in B, k_{3} \in A$ ).

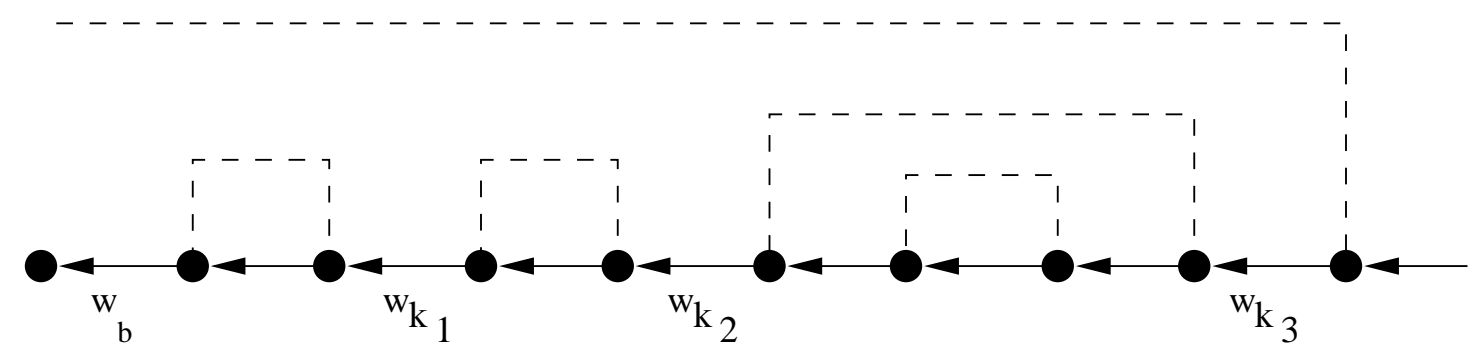

Fig. 8

Proof of Lemma 3.10. We shall consider $r=0$, the general case is similar. Let $Y(z):=$ $\left(Y_{z} f\right)(0)$. Define

$$
f^{*}(Q):=\int_{S^{d-1}} f\left(-Q^{1 / 2} \omega\right) d \omega
$$

where $Q \geq 0$ and the integration is on the unit sphere $S^{d-1}$ with respect to the normalized surface measure. Clearly $f^{*}$ is bounded, smooth away from 0 , it decays fast at infinity and 
its derivative has a $Q^{-1 / 2}$ singularity at $Q=0$. We have

$$
Y(z)=\int_{0}^{\infty} \frac{f^{*}(Q)}{z-Q} Q^{\frac{d}{2}-1} d Q .
$$

(i) For the proof of (3.64) we assume that $\alpha=\operatorname{Re} z \geq 0$; the case $\alpha<0$ is similar but easier. We split the integration as follows

$$
Y(z)=\int_{J} \frac{f^{*}(Q)}{z-Q} Q^{\frac{d}{2}-1} d Q+\int_{J^{c}} \frac{f^{*}(Q)}{z-Q} Q^{\frac{d}{2}-1} d Q,
$$

where $J:=[\max (0, \alpha-1), \alpha+1]$ and $J^{c}=[0, \infty) \backslash J$. On the set $J^{c}$ we use the estimate $|z-Q| \geq 1$, hence this integral is bounded by (const.) $\|f\|_{2 d, 2 d}$. On the set $J$ we use the identity

$$
\int_{J} \frac{f^{*}(Q)}{z-Q} Q^{\frac{d}{2}-1} d Q=\int_{J} \frac{g(Q)-g(\alpha)}{\alpha-Q+i \eta} d Q+g(\alpha) \int_{J} \frac{1}{\alpha-Q+i \eta} d Q
$$

with

$$
g(Q):=f^{*}(Q) Q^{\frac{d}{2}-1},
$$

The function $g^{\prime}(Q)$ inherits the $Q^{-1 / 2}$ singularity at the origin from the derivative of $f^{*}$. We easily obtain for $|\alpha-Q| \leq 1$ that

$$
g(Q)-g(\alpha) \leq \text { (const. })|| f \|_{2 d, 2 d}|\sqrt{Q}-\sqrt{\alpha}| .
$$

Hence the first term in (6.20) is estimated

$$
\left|\int_{J} \frac{g(Q)-g(\alpha)}{\alpha-Q+i \eta} d Q\right| \leq \text { (const.) }\|f\|_{2 d, 2 d} \int_{J} \frac{1}{\sqrt{\alpha}+\sqrt{Q}} d Q \leq \text { (const.) }\|f\|_{2 d, 2 d} .
$$

The second term in (6.20) is essentially explicit:

$$
g(\alpha) \int_{J} \frac{1}{\alpha-Q+i \eta} d Q=g(\alpha) \int_{J} \frac{(\alpha-Q)+i \eta}{(\alpha-Q)^{2}+\eta^{2}} d Q
$$

The imaginary part of the integrand is uniformly bounded. The integral

$$
\int_{\max (0, \alpha-1)}^{\alpha+1} \frac{\alpha-Q}{(\alpha-Q)^{2}+\eta^{2}} d Q
$$

is zero if $\alpha \geq 1$ by symmetry. For $\alpha<1$

$$
\left.\int_{\max (0, \alpha-1)}^{\alpha+1} \frac{\alpha-Q}{(\alpha-Q)^{2}+\eta^{2}} d Q=\int_{2 \alpha}^{\alpha+1} \frac{\alpha-Q}{(\alpha-Q)^{2}+\eta^{2}} d Q \leq \text { (const.) }\right)|\log \alpha|
$$


Considering that $g(\alpha) \sim \alpha^{\frac{d}{2}-1}$ for small $\alpha$, we see that the second term in $(6.20)$ is bounded for $d \geq 3$, and has a logarithmic singularity for $d=2$. This concludes the proof of part (i).

(ii) For the proof of (3.66) and (3.67), notice that

$$
\frac{1}{z-Q}=-\frac{d}{d Q} \log (z-Q) \text {. }
$$

Since $\operatorname{Im} z>0$ and $Q$ is real, we always evaluate the logarithm on the upper half plane. So we can choose any branch of the logarithm and fix it. Integration by parts from (6.19) gives

$$
Y(z)=-f^{*}(0) \log z+\int_{0}^{\infty} \log (z-Q) F(Q) d Q
$$

if $d=2$, and

$$
Y(z)=\int_{0}^{\infty} \log (z-Q) F(Q) d Q
$$

if $d \geq 3$, with

$$
F(Q):=\frac{d}{d Q}\left(f^{*}(Q) Q^{\frac{d}{2}-1}\right)
$$

Notice that $F(Q)$ is smooth away from the origin, it has a strong decay at infinity and it has a $Q^{-1 / 2}$ singularity at $Q=0$. Hence

$$
\left|Y(z)-Y\left(z^{\prime}\right)\right| \leq\left\|f^{*}\right\|_{\infty}\left|\log z-\log z^{\prime}\right|+\left|\int_{0}^{\infty}\left(\log (z-Q)-\log \left(z^{\prime}-Q\right)\right) F(Q) d Q\right|
$$

and the first term is present only in $d=2$. For the first term we use the following estimate which can easily be checked

$$
\left.\left|\log z-\log z^{\prime}\right| \leq \text { (const.) }\right)\left|z-z^{\prime}\right|\left(\frac{1}{|z|}+\frac{1}{\left|z^{\prime}\right|}\right) .
$$

We rewrite the second term

$$
\begin{aligned}
\left|\int_{0}^{\infty}\left(\log (z-Q)-\log \left(z^{\prime}-Q\right)\right) F(Q) d Q\right| & =\left|\int_{\Gamma\left(z, z^{\prime}\right)} d \xi \int_{0}^{\infty} \frac{F(Q)}{\xi-Q} d Q\right| \\
& \leq \int_{\Gamma\left(z, z^{\prime}\right)} d|\xi| \int_{0}^{\infty} \frac{|F(Q)|}{|\xi-Q|} d Q
\end{aligned}
$$

where $\Gamma\left(z, z^{\prime}\right)$ is any path in the upper half plane that connects $z, z^{\prime}$. Here $d|\xi|$ is the arclength measure. It is an easy exercise to show that for $\operatorname{Im} \xi>0$

$$
\left.\int_{0}^{\infty} \frac{|F(Q)|}{|\xi-Q|} d Q \leq \text { (const. }\right)|\xi|^{-\frac{1}{2}}\|f\|_{2 d, 2 d}
$$


using the properties of $F$ listed above. We choose the path $\Gamma\left(z, z^{\prime}\right)$ that goes from $z=\alpha+i \eta$ to $\alpha^{\prime}+i \eta$ then to $z^{\prime}=\alpha^{\prime}+i \eta^{\prime}$ along straight line segments (recall that $\eta^{\prime} \leq \eta \leq 1 / 2$ ). The integral of the right hand side of (6.22) along this path gives

$$
\left.\int_{\Gamma\left(z, z^{\prime}\right)} d|\xi| \int_{0}^{\infty} \frac{|F(Q)|}{|\xi-Q|} d Q \leq \text { (const. }\right)\left|z-z^{\prime}\right||\eta|^{-1 / 2}\|f\|_{2 d, 2 d}
$$

Combining these estimates we obtain (3.66) and (3.67).

Acknowledgement. We would like to thank H. Spohn for his several comments and discussions on this project. Part of this work was done during the time when L. E. visited the Erwin Schrödinger Institute in Vienna and when both authors visited the Center of Theoretical Sciences in Taiwan. We thank them for the hospitality and the support of this work.

\section{References.}

1 C, Boldrighini, L. Bunimovich, Y.Sinai: On the Boltzmann equation for the Lorentz gas, J. Stat. Phys. 32, 477-501, (1983).

2 D. Durr, S. Goldstein and J. Lebowitz: Asymptotic motion of a classical particle in a random potential in two dimensions: Landau model. Commun. Math. Phys. 113, 209$230(1987)$.

3 L. Erdős and H.-T. Yau: Linear Boltzmann equation as scaling limit of quantum Lorentz gas, Advances in Differential Equations and Mathematical Physics. Contemporary Mathematics 217, 137-155 (1998).

4 R. Esposito, M. Pulvirenti and A. Teta: The Boltzmann equation for a one-dimensional quantum Lorentz gas, Preprint (1998).

5 G. Gallavotti: Rigorous theory of the Boltzmann equation in the Lorentz gas. Nota inteerna n. 358, Univ. di Roma (1970).

6 T. G. Ho, L. J. Landau and A. J. Wilkins: On the weak coupling limit for a Fermi gas in a random potential, Rev. Math. Phys. 5, 209-298 (1992).

7 H. Kesten, G. Papanicolaou: A limit theorem for stochastic acceleration, Commun. Math. Phys. 78, 19-63 (1980).

8 H. Spohn: Derivation of the transport equation for electorns moving through random impurities, J. Stat. Phys., 17, 385-412 (1977). 
9 H. Spohn: The Lorentz process converges to a random flight process. Commun. Math. Phys. 60, 277-290 (1978).

László Erdős: School of Mathematics, Georgiatech, ATlantA, GA-30332-0160, USA

E-mail address: lerdos@math.gatech.edu

Horng-Tzer Yau: Courant Institute, New York University, 251 Mercer Street, New York NY 10012, USA.

E-mail address: yau@cims.nyu.edu 